\title{
39. PRE-LEG 55 SITE SURVEY GEOPHYSICAL DATA FROM R/V S. P. LEE CRUISE LEE8-76-NP
}

\author{
G. Brent Dalrymple and H. Gary Greene, U. S. Geological Survey, Menlo Park, California \\ Byron D. Ruppel and Theodore E. Bear, U. S. Geological Survey, Seattle, Washington \\ and \\ David A. Clague, Middlebury College, Middlebury, Vermont
}

\section{INTRODUCTION}

Cruise LEE8-76-NP began with the departure of the U. S. Geological Survey Research Vessel S. P. Lee from Adak Island, Alaska, at 0204 GMT on 29 September 1976. The intended cruise track was southwest from Adak to Tenchi Seamount at the northern end of the Emperor Seamount chain, southward along the Emperor Seamounts to Kōkō Seamount, and westward from Kōkō along the Hawaiian Ridge to the cruise termination port of Honolulu, Oahu. The cruise was scheduled to arrive in Honolulu at 2200 GMT on 21 October.

The main purpose of the cruise was to obtain geophysical data and samples of volcanic rock from the volcanoes that form the Emperor Seamounts. These data and samples were needed for an ongoing experiment, which included the proposed DSDP Leg 55 drilling in the Emperor Seamounts, designed to test the hot-spot hypothesis for the origin of the Hawaiian-Emperor volcanic chain (see Jackson et al, this volume). A secondary purpose of the cruise was to conduct site surveys for Leg 55 drilling on Suiko Seamount, a potential reentry target, and on several other major seamounts in the chain that were potential sites for single-bit holes.

\section{OPERATIONS AND WEATHER}

On departure from Adak, we were operating a hullmounted 3.5-kHz echo sounder, a 3-axis LaCoste and Romberg sea gravity meter (S-53), a Geometrics 801G marine proton precession gradiometer, and a singlechannel $160-\mathrm{kJ}$ continuous sub-bottom profiler. The energy sources for the profiler were four sparker ladders streamed outboard from booms located slightly aft of amidships. The reflected sparker signals were received by a Teledyne high-speed hydrophone streamer, filtered with a 36- to $98-\mathrm{Hz}$ bandpass filter, and recorded on a Raytheon facsimile recorder. Navigation control was from a satellite-based integrated navigation system using a Canadian Marconi satellite receiver with gyroerected acoustic Doppler speed measurement. Electronic problems with the $12-\mathrm{kHz}$ echo sounder prevented its operation, except for a short period at the beginning of the cruise. A Uniboom profiler with four hull-mounted transducers was used for a short time while crossing Suiko Seamount.

Shortly after leaving Adak on a southwesterly course toward Tenchi Seamount, we encountered rising winds and seas generated by a storm moving northeastward toward the western Aleutians at a speed of 20 to 30 knots (Dalrymple, 1977). By 1200 GMT on 30 September, the storm center was only 230 miles to our west. The captain changed the heading to a more southerly course and reduced the speed to ease the motion of the vessel. We secured the scientific gear because the data were being severely degraded by the ship's motion and by sea noise. We raised the sparker booms to a $45^{\circ}$ angle to prevent them from dipping into the seas as the Lee rolled, but left the sparker ladders, hydrophone streamers, and gradiometer sensors in the water because we judged retrieval to be too hazardous and the forecasts indicated that the weather would not get much worse. By 0100 GMT on 1 October, however, the Lee was hove-to in winds of 55 to 60 knots and 25 -foot seas. During the next 27 hours, the Lee was battered by winds that were consistently over 55 knots, with gusts to 70 knots, and seas with significant wave heights of 35 to 40 feet; the largest waves reached 60 feet. The winds began to abate by 0900 GMT on 2 October, and by 0200 GMT the seas had decreased to 20 feet and the winds to 20 knots. We recovered and repaired the remaining overboard scientific gear and were underway, with the scientific systems operating, by 0400 GMT, 4 October.

During the storm, the gradiometer sensors and the four sparker ladders were lost, their cables sheared by the propeller. A spare magnetometer streamer was used to replace the lost gradiometer sensors, and the remains of the four sparker cables were spliced to permit operation of two $40-\mathrm{kJ}$ Sparker ladders ( $80 \mathrm{~kJ}$ total). The most serious consequence of the storm was that the structural support for a 4-ton, multi-channel hydrophone reel mounted on the fantail was severely damaged. The reel was secured by cables and turnbuckles, but still threatened the safety of the ship. We therefore had to make substantial changes in the cruise plan. We cancelled all plans for dredging, and altered the ship's track to intersect the Emperor Seamounts at a point just north of Suiko Seamount, the prime site-survey objective. Because the damaged hydrophone reel still posed a hazard, the winds and seas were yet only marginally acceptable for underway operations, and the central Emperor Seamounts lie almost directly in the path of fall and winter North Pacific storms, we reduced the survey lines to single seamount crossings on a continuing southerly course. This drastically modified cruise plan offered the only chance to accomplish the basic Leg 55 site survey goals without further endangering the ship and crew. From Suiko, we followed a generally southerly course to Kōkō Seamount, then a southeasterly course to Midway 
Island, where we put in at 1940 GMT, 12 October, for more substantial repairs to the damaged deck-mounted equipment.

After three and a half days at Midway for repairs, we left at $0200 \mathrm{GMT}, 16$ October, and proceeded along the Hawaiian chain toward Oahu on an easterly course. The track was chosen to maximize the number of Hawaiian seamount crossings without jeopardizing the scheduled arrival time in Honolulu. On October 17, we made two dredge attempts at latitude $25^{\circ} 55^{\prime} \mathrm{N}$, longitude $171^{\circ}$ $44^{\prime} \mathrm{W}$, on the volcano that underlies Laysan Island. The purpose of the dredging was to obtain samples for chemistry and $\mathrm{K}-\mathrm{Ar}$ dating to fill a data gap in the Hawaiian chain. The first dredge yielded 16 wellrounded cobbles of volcanic rock totaling approximately $11.6 \mathrm{~kg}$. On the second attempt, a weld on the tensiometer support broke, disabling the deep-sea winch and resulting in the loss of a chain-bag dredge and approximately 300 meters of cable. The Lee proceeded from Laysan to Honolulu and arrived on schedule at 1900 GMT, 21 October.

\section{DATA COLLECTED}

The Lee was at sea for 466 hours, which included 24.5 hours of transit, 2.4 hours on dredge station, and 439.1 hours on 34 geophysical tracklines with a cumulative trackline distance of $6288 \mathrm{~km}$. The track of the Lee down the Emperor Seamounts and along the Hawaiian Ridge is shown in Figures 1 through 4. The 34 individual tracklines, most of which are indicated on the figures, are numbered $1 \mathrm{~A}$ through $1 \mathrm{E}, 2$, and 14 through 41 . A summary of the types and amounts of data collected on the cruise is given in Table 1 and the Cruise Data Index (Table 2).

The bathymetric, magnetic, gravity, and navigation data collected on the cruise are tabulated in Table 3 at 5 -minute intervals. Navigation data have been smoothed to give the best estimate of the ship's position at the indicated times. Water depths are based on an average velocity of sound in seawater of $1500 \mathrm{~m} / \mathrm{s}$. The tabulated magnetic anomaly has the 1975 IGRF removed. The Bouguer gravity was calculated with reference to the 1967 ellipsoid using a crustal density of $2.67 \mathrm{~g} / \mathrm{cm}^{3}$ and a sea water density of $1.03 \mathrm{~g} / \mathrm{cm}^{3}$. No regional trends were removed from the profiles but, in general, a gently concave regional trend can be seen in the individual profiles. Recomputation of the Bouguer gravity at densities other than $2.67 \mathrm{~g} / \mathrm{cm}^{3}$ indicates that the average density of the seamounts is probably less than $2.5 \mathrm{~g} / \mathrm{cm}^{3}$, an observation that is consistent with findings from the $\mathrm{Ha}-$ waiian Islands and from other volcanoes in the Pacific (Malahoff, 1969).

\section{PROFILES ACROSS THE EMPEROR SEAMOUNTS}

Eight major seamounts of the Emperor chain were crossed during the cruise. The seismic reflection, bathymetric, magnetic, and gravity profiles are shown in Figures 5 through 12 with line drawings of the seismic reflection profiles and geologic interpretations. The locations of the profiles are indicated on Figures 1 and 2.
The eight Emperor seamounts crossed by the Lee and discussed here are, from north to south, Seamount "A" (Northern Suiko), Suiko, Seamount "B", Yōmei (Seamount " $C$ " of Greene et al., 1978), Nintoku, Jingū, Ōjin, and Kōkō. All have a similar morphology, being flat-topped or gently domed and covered with carbonate reef or bank sediments. ${ }^{1}$ But the seamounts have subtle differences in their tectonic and sedimentologic histories, recorded in the subsurface geology as seen in the geophysical profiles discussed here and in the drill hole data collected aboard Glomar Challenger on Suiko (Site 433), Yōmei (Site 431), Nintoku (Site 432), and Ōjin (Site 430) seamounts (Greene, this volume; McKenzie et al., this volume). The following is a brief discussion of the morphology of each seamount.

\section{Seamount "A" (Northern Suiko)}

This seamount (Figure 5) is situated at latitude $45^{\circ} 30^{\prime} \mathrm{N}$, longitude $170^{\circ} \mathrm{E}$. It is flat-topped and narrow, with a north-south elongation. It is $46 \mathrm{~km}(25 \mathrm{n}$. mi.) wide, $93 \mathrm{~km}(50 \mathrm{n}$. mi.) long, and has a crest area of about $4300 \mathrm{~km}^{2}$. The crest of the seamount lies at a depth of 1520 meters and stands approximately 5400 meters above the flat sea floor to the east and $\mathbf{5 8 5 0}$ meters above the sea floor to the west. The seamount appears to be a single volcanic structure covered with sedimentary deposits. The northeast flank is steeper $\left(\sim 17^{\circ}\right)$ than the southwest $\left(\sim 12^{\circ}\right)$, where much more slump debris appears to have collected near the base.

The seismic reflection profile indicates that a reef cap overlies the volcanic structure. Acoustic data show that fringing reefs enclose a central lagoonal deposit that overlies older reef material. The entire reef assemblage overlies an eroded, flat, volcanic surface faulted near the center of the seamount and buried beneath about 25 meters of reef material. The volcanic surface crops out just below the crest's edge in approximately 1800 meters of water. The surface of the central lagoonal deposits is flat, and the depths to the fringing reefs on either side of the seamount are equal $(1575 \mathrm{~m})$.

Two faults that displace the volcanic surface are the only apparent structural features. A normal fault offsets the volcanic surface by 75 meters, east side down, beneath the lagoonal deposits overlying the central part of the seamount. The other, smaller fault appears to displace the volcanic surface near the east edge of the seamount.

The residual magnetic anomaly profile across the seamount shows the influence of the faulted volcanic surface. The profile also indicates that "Northern Suiko" is reversely magnetized, as is Suiko to the south (Koda-

\footnotetext{
${ }^{1}$ Leg 55 drilling has shown that the shallow-water carbonate sediments atop these seamounts are primarily bryozoan-algal banks rather than coral-algal reefs (McKenzie et al., this volume; Hagn et al., this volume). The seismic profiles (Figures 5 through 12) show, however, some acoustically opaque structures that may be discontinuous reefs. For simplicity, we use the terms reef cap, reef assemblage, etc., to include shallow-water carbonate banks and associated sedimentary deposits.
} 


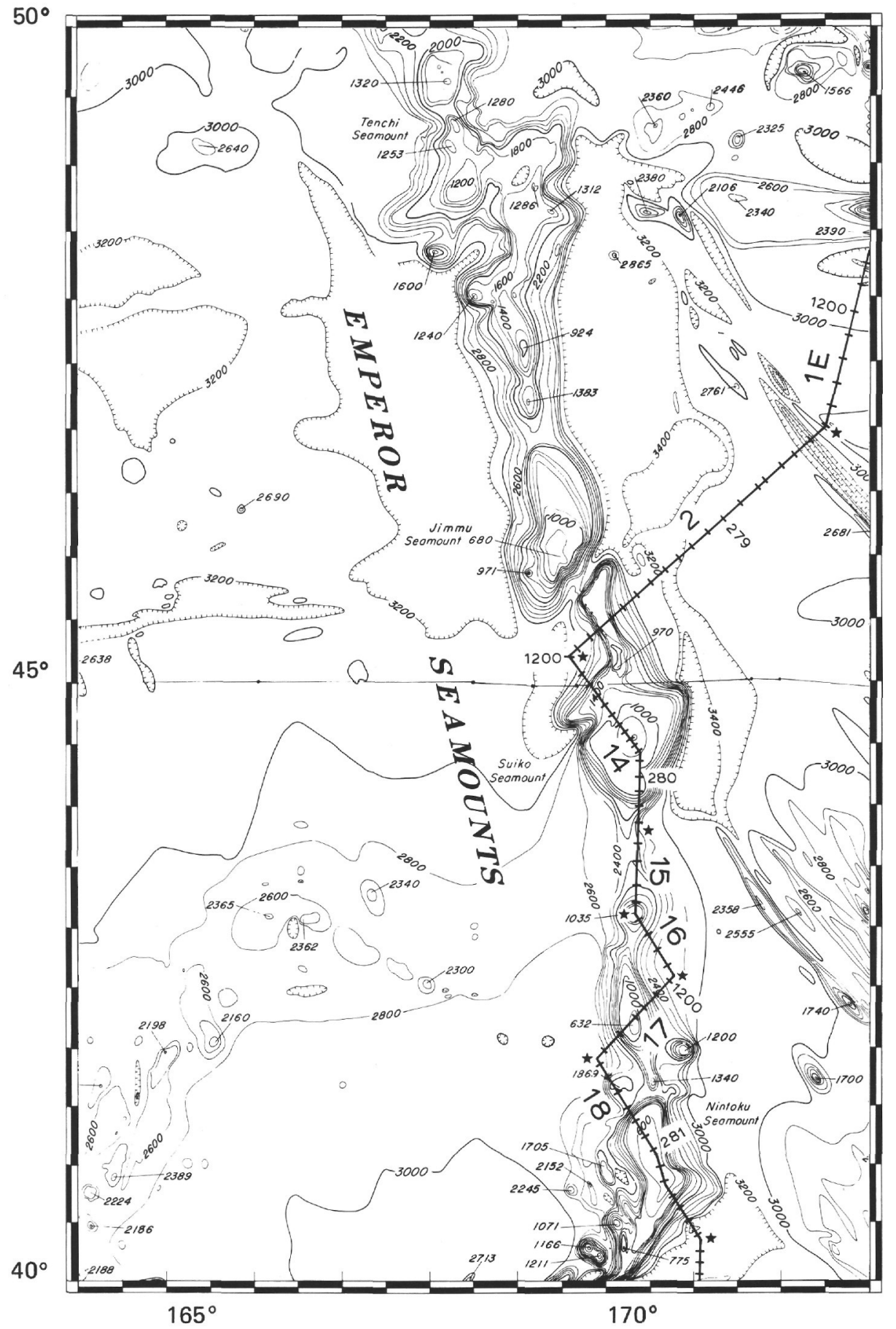

Figure 1. Track of $R / V$ S. P. Lee in the northern Emperor Seamounts, Cruise LEE8-76-NP. The ends of the numbered tracklines are indicated by stars. Time tics are hourly and labeled with the day number at 0000 GMT and with 1200 at noon GMT. Bathymetry after Chase et al. (1970); depths in fathoms.

ma et al., 1978; Kono, this volume). The most striking change in the magnetic profiles is at $0900 \mathrm{Z}$, were a 600 gamma negative anomaly reflects the large normal fault.

The inverted Bouguer profile over the flat-topped structure indicates an average density lower than 2.67 $\mathrm{g} / \mathrm{cm}^{3}$. Even allowing for artifacts of reduction, none of the structural features evident in the seismic and mag- netic data are evident in the gravity profile. Depth to the ridge and the narrowness of the feature apparently obscure the detail.

\section{Suiko Seamount}

Suiko Seamount (Figure 6) is at $44^{\circ} 33^{\prime} \mathrm{N}$ latitude and $170^{\circ} 20^{\prime} \mathrm{E}$ longitude. It is a gently domed guyot, elon- 


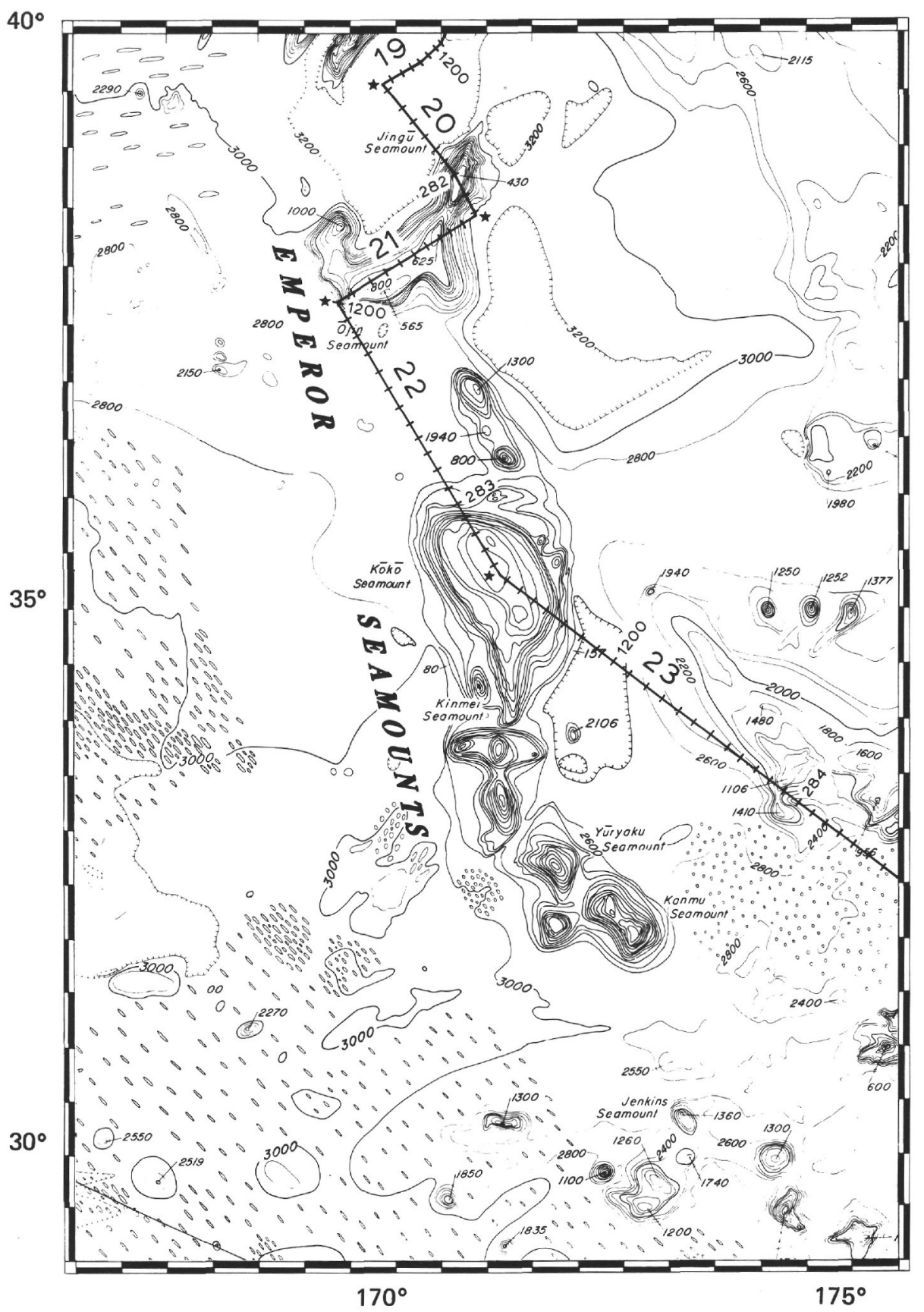

Figure 2. Track of $R / V$ S. P. Lee in the southern Emperor Seamounts, Cruise LEE8-7-76-NP. Symbols as in Figure 1. Bathymetry after Chase et al. (1970); depths in fathoms.

gated in a north-south direction, $124 \mathrm{~km}(67 \mathrm{n} . \mathrm{mi}$. wide, and $370 \mathrm{~km}(200 \mathrm{n} . \mathrm{mi}$.) long. The flat top covers an area of about $46,000 \mathrm{~km}^{2}$, and the crest lies at a depth of 1068 meters. Suiko rises 1360 meters above the sea floor to the north and approximately 4100 meters above the sea floor to the south. Its northern flank is steeper ( $\sim 15^{\circ}$ slope), and contains more slump debris than the south flank $\left(\sim 5^{\circ}\right.$ slope).

The seismic reflection profile across Suiko indicates a cover of reef material that ovelies an irregular volcanic surface. The seamount appears to have a large central bioherm surrounded by fringing reefs and ponded lagoonal sediments. The tops of the reefs lie at a depth of 1450 meters on the north side and 2130 meters on the south. The distal perimeter of the seamount is 1910 meters deep on the north and 1790 meters deep on the south, suggesting a slight northward tilt.

Sediment thickness varies from less than 30 meters near the crest, where volcanic rocks crop out, to over 500 meters in the north lagoon. The upper limits of ex- 


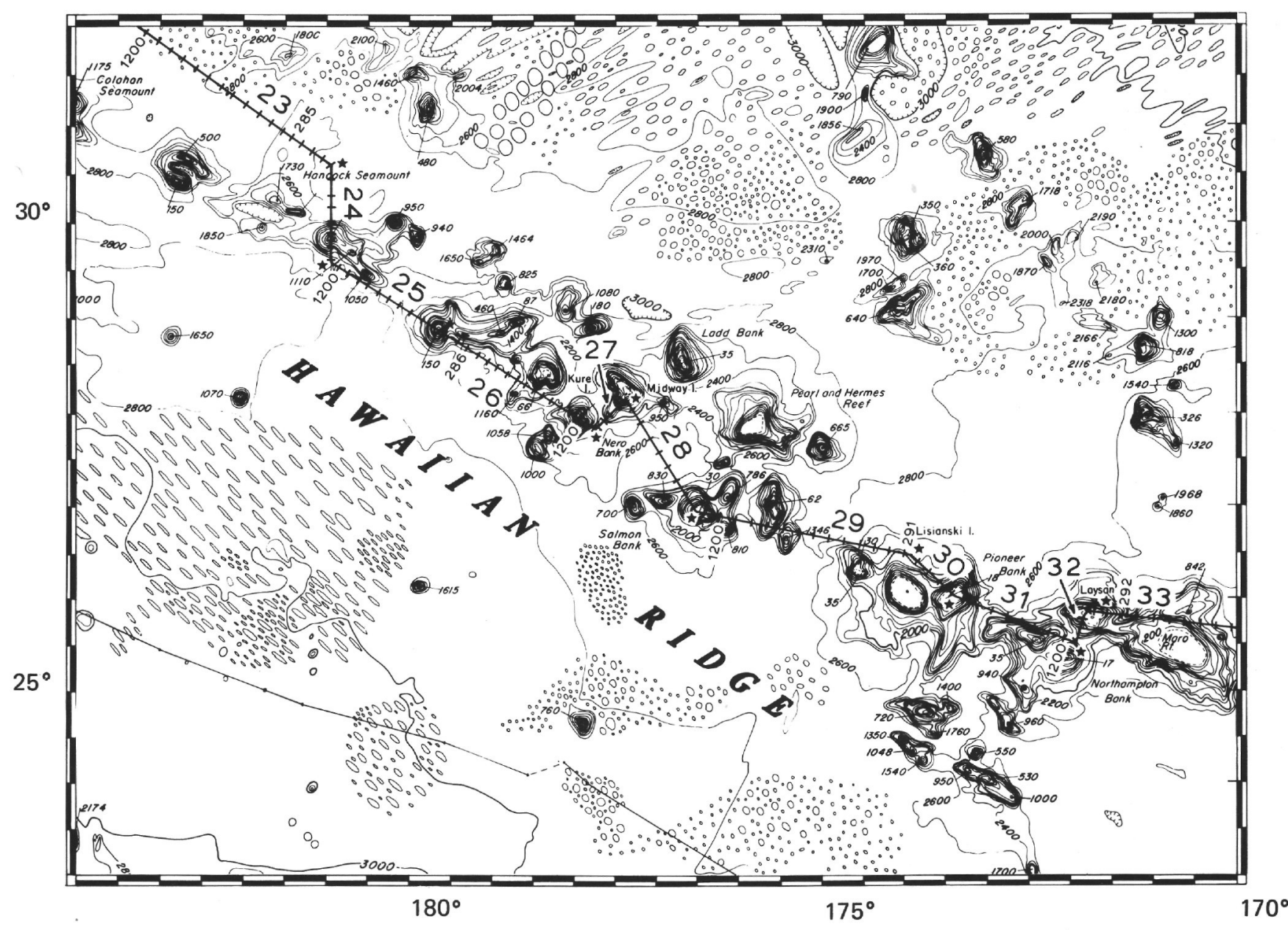

Figure 3. Track of R/V S. P. Lee along the western Hawaiian Ridge, Cruise LEE8-76-NP. Symbols as in Figure 1. Bathymetry after Chase et al. (1970); depths in fathoms.

posed volcanic rocks on the flanks of the seamount occur at 2590 meters on the north and 2360 meters on the south.

The structure of the seamount is dominated by faults that have offset the volcanic surface and sedimentary strata on the north side. Some faults appear to offset the sea floor, and may have moved recently. The basin that contans the lagoonal deposits is structurally controlled by these faults. Sediments within the basin are flatlying, except near the faults, where drag folding has occurred. An unconformity evident in the profile is offset by several of these faults.

Magnetic data for Suiko Seamount are limited to a short segment across the north edge. This profile shows a 500 -gamma negative anomaly and a 900 -gamma positive anomaly at about $1630 \mathrm{Z}$. There is nothing in either the seismic or gravity profiles to indicate the source of these anomalies. The pronounced magnetic relief in this water depth suggests a high magnetic contrast within the volcanic rocks of the seamount.

The ponded lagoonal sediments are reflected in the gentle decrease in the Bouguer anomaly. The structural discontinuity at $1800 \mathrm{Z}$ is obscured by an imperfect Eotvos correction, but the difference in slope between the left and right sides of the profile is consistent with the interpretation of an abruptly bounded basin.

\section{Seamount "B"}

Seamount " $B$ "' (Figure 7), located at $43^{\circ} \mathrm{N}$ latitude, $170^{\circ} 25^{\prime} \mathrm{E}$ longitude, is an elongate guyot capped at its crest with biogenic bank or reef deposits. It is $74 \mathrm{~km}(40$ n. mi) wide, $185 \mathrm{~km}$ (100 n. mi.) long, and about 13,700 $\mathrm{km}^{2}$ in area. Depth to the crest and reef is $\mathbf{1 5 2 0}$ meters, and the depths of the upper break in slope at the north and south are 2050 meters and 2025 meters, respectively. The elevation of the crest above the sea floor to the north is 5080 meters. The north slope is inclined $8^{\circ}$ and the south slope $12^{\circ}$. There is relatively little slump debris at the base of slope.

The seismic reflection profile shows a thin sedimentary cover overlying the domed volcanic surface. These sediments appear to be depositional terraces surrounding the central reef. A smaller, fringing reef on the south side has ponded the terrace deposits, which apparently consist of biogenic detritus. Near the seamount's edge these terrace deposits are over 150 meters thick. The upper limits of volcanic rock exposures on the flanks are 2360 meters on the north and 2305 meters on the south. 


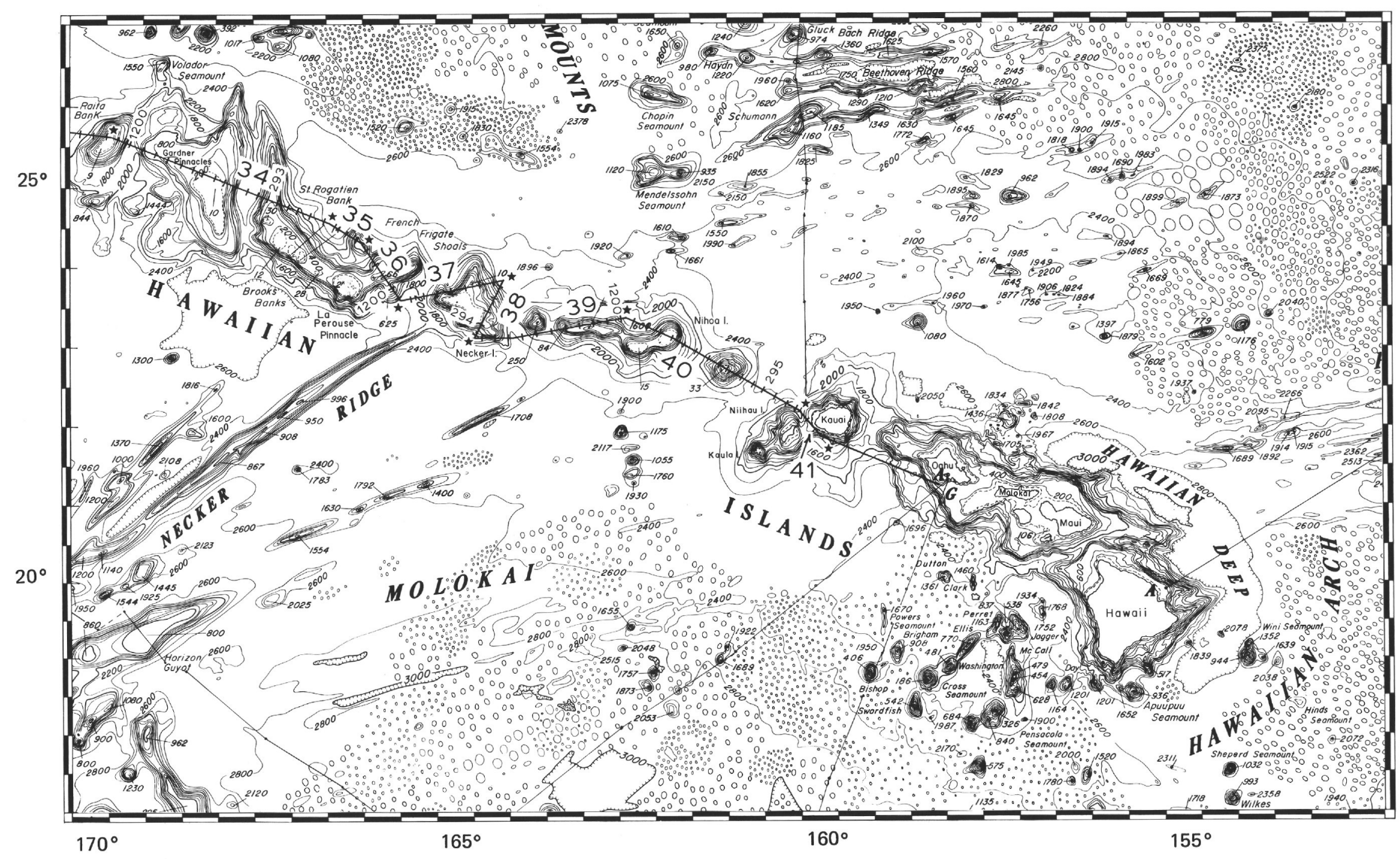

Figure 4. Track of R/V S. P. Lee along the eastern Hawaiian Ridge, Cruise LEE8-76-NP. Symbols as in Figure 1. Bathymetry after Chase et al. (1970); depths in fatroms. 
TABLE 1

Geophysical Data Summary for Cruise LEE8-76-NP

\begin{tabular}{lrrr}
\hline Type of Data & $\begin{array}{c}\text { Trackline } \\
(\mathrm{km})\end{array}$ & $\begin{array}{c}\text { Recording } \\
\text { Time } \\
\text { (hours) }\end{array}$ & $\begin{array}{c}\text { Trackline } \\
\text { Coverage } \\
(\%)\end{array}$ \\
\hline Single-Channel Reflection & 5599.5 & 334.1 & 89.1 \\
$\quad$ Profiler & 4609.3 & 355.0 & 73.3 \\
3.5-kHz & 240.2 & 12.9 & 3.8 \\
12-kHz Bathymetry & 28.8 & 2.9 & 0.5 \\
Uniboom Profiler & 8.7 & 0.7 & 0.1 \\
Sonobuoy & 4074.4 & 231.3 & 64.8 \\
Magnetometer & 603.9 & 56.6 & 9.6 \\
Magnetometer/Gradiometer & 5340.0 & 344.0 & 84.9 \\
Gravity Meter & & & \\
\hline
\end{tabular}

\section{Yōmei Seamount}

Yōmei Seamount (Figure 8), located at $42^{\circ} 20^{\prime} \mathrm{N}$ latitude and $170^{\circ} 20^{\prime} \mathrm{E}$ longitude, consists of two volcanic peaks surrounded by flat depositional platforms. Three down-dropped blocks exist on the north flank. Depths of the crests are 980 meters and 1125 meters. The seamount rises 5290 meters above the sea floor to the west, where its flank has a $14^{\circ}$ slope covered with slump deposits. Yomei is elongated in a north-south direction, with a width of $56 \mathrm{~km}(30 \mathrm{n} . \mathrm{mi}$.), a length of $93 \mathrm{~km}$ (50 n.mi.), and an area of about $5150 \mathrm{~km}^{2}$.

The seismic reflection profile across Yōmei indicates that the flat erosional volcanic platform surrounding the volcanic peaks is composed of lagoonal deposits. Fringing reefs along the top edge of the seamount are the outer barriers to lagoonal deposits. The upper limits of volcanic rock outcrops on the flanks are 1690 meters on the north and 1790 meters on the south. Thicknesses of the sediments vary from $\mathbf{7 5}$ meters on the south to 100 meters on the north.

The north flank of the seamount is faulted into three distinct, flat-topped, stepped-down blocks. These steps are displaced along normal faults and are capped with depositional terrace deposits. Depths to the surfaces of these terraced biocks are 1800,1912 , and 2025 meters.

Two positive gravity anomalies are apparent above a regional downwarp. The one between $1330 \mathrm{Z}$ and $1500 \mathrm{Z}$ is related to the volcanic outcrop on the seamount crest. This anomaly falls off to the southwest, as the reef is approached. The second anomaly occurs between $1530 \mathrm{Z}$ and $1645 \mathrm{Z}$ over what may be a downdropped block on this flank of the seamount.

\section{Nintoku Seamount}

Nintoku Seamount (Figure 9), at latitude $41^{\circ} 05^{\prime} \mathrm{N}$ and longitude $170^{\circ} 30^{\prime} \mathrm{E}$, is a guyot elongated northwest-southeast. It is $185 \mathrm{~km}(100 \mathrm{n}$. mi.) long and 148 $\mathrm{km}(80 \mathrm{n}$. mi.) wide, with a surface area of about 27,400 $\mathrm{km}^{2}$. The crest is 850 meters deep, and depths to the north and south top edges are 1400 meters and 1350 meters, respectively. This seamount has gentler slopes than the other seamounts surveyed. Its north flank has an inclination of $6^{\circ}$ and its south flank $10^{\circ}$. The seamount rises approximately 5600 meters from the ocean floor on the north and 6410 meters on the south.
A thin ( $80 \mathrm{~m}$ average) layer of reef sediment overlying a flat, gently undulating volcanic surface is evident in the seismic reflection profile. Volcanic rocks locally project above the sediment, and are exposed on the sea floor. The upper limits of the flank exposures of the volcanic rocks are at depths of 1680 meters on the north and 2135 meters on the south. Fringing reefs are present along the top edge of the seamount just above the volcanic surface. These reefs occur at depths of 1575 meters on the north and 2025 meters on the south. Depositional terraces blanket the outer margin of the seamount's top, and vary in thickness from about 170 meters on the north to nearly 450 meters on the south. Much slump debris covers the flank of this seamount, and ponded, well-bedded sediments have accumulated behind basement ridges along its south slope.

The gentle positive gravity anomaly reflects the massive, domed, thinly veneered structure.

\section{Jingū Seamount}

Jingū Seamount (Figure 10) is situated at $38^{\circ} 45^{\prime} \mathrm{N}$ latitude, $171^{\circ} 12^{\prime} \mathrm{E}$ longitude. It is steep, oval in shape $(59 \mathrm{~km} \times 74 \mathrm{~km})$, elongated north-south, and flattopped, with an upper surface area of only about 4370 $\mathrm{km}^{2}$. Its crest is 845 meters deep, and the depth to both its northwest and southeast top edges is 860 meters. The northwest flank has a $16^{\circ}$ inclination and the southeast flank $20^{\circ}$. The seamount rises 6300 meters above the flat sea floor to the north and $\mathbf{5 7 4 0}$ meters to the south.

The seismic reflection profile across the seamount shows a very thin layer of terrace material overlying a nearly flat volcanic surface. These terrace deposits range in thickness from about 75 meters near the center of the seamount to nearly 180 meters on the southeast edge. Comparatively little slump material has accumulated along the seamount's flanks. To the southeast, a hummock-topped volcanic peak projects above the flank of the seamount.

The main peak and volcanic peak on the southeast flank both produce slight positive gravity anomalies above a regional downwarping.

\section{Ōjin Seamount}

Ōjin Seamount (Figure 11), at $38^{\circ} \mathrm{N}$ latitude, $170^{\circ}$ $30^{\prime} \mathrm{E}$ longitude, lies just south of Jingū Seamount, and is separated from it by only a shallow saddle. Its shape is generally elongated in an east-west direction, with an average width of $37 \mathrm{~km}(20 \mathrm{n}$. mi.), length of $130 \mathrm{~km}$ (70 n. mi.), and a top surface area of about $4800 \mathrm{~km}^{2}$. The seamount is flat-topped or gently downbowed, with a crestal depth of 1575 meters and edge depths of 1070 meters on the west and 1010 meters on the north. It has gentle flanks that have a $12^{\circ}$ west slope and a $8^{\circ}$ east slope. The east and west sides of the seamount are elevated 5060 and 5740 meters, respectively, above the flat sea floor.

The seismic reflection profile shows a complex reef morphology. Lagoonal deposits bounded by large reefs or reef-like structures appear to partially fill the central depression. Depths to the reefs are 1125 meters on the west and 1160 meters on the east. Thickness of the reefs 
TABLE 2

Cruise Data Table for LEE8-76-NP, R/V S.P. Lee (includes navigation fixes for data entries)

\begin{tabular}{|c|c|c|c|c|c|c|c|c|c|c|c|}
\hline & & CRUISE/D & ATA INFO & DATA & PERSONN & L,PORTS,EQU & UI PMENT & WATER & & & \\
\hline JUL. & TIME & RECORD. & SEGNCE & STATUSI & OESCRIP & ION OR: & & DEPTH & LATI & TUDE & LONGITUDE \\
\hline DAY & (GMT) & MEDIUM & NUMBER & INSTITUTE & LINE\# & STA.ISHOT & $P T_{.} \#$ & UNCOR. & $D E G$ & M I N & DEG MIN \\
\hline
\end{tabular}

CRUISE DATES AND PORT STOPS

$\begin{array}{rrlll}273 & 24.0 & \text { CRUISE } & \text { START } & \text { LV ADAK, AK ST CRUZ } \\ 286 & 1940.0 & \text { IN PORT } & \text { ARRIVE } & \text { ARRIVE MIDWAY IS. } \\ 290 & 20.0 & \text { IN PORT } & \text { LEAVE } & \text { LEAVE MIDWAY IS. } \\ 295 & 190.0 & \text { CRUISE } & \text { END } & \text { AR HONOLULU ED CRUZ }\end{array}$

PERSONNEL LIST

$\begin{array}{llll}272 & 0 & 0.0 & \text { SHIP CAPTAIN } \\ 272 & 0 & 0.0 & \text { CHIEF ENGINEER }\end{array}$

$\begin{array}{llll}272 & 0 & 0.0 & \text { CHIEF MATE } \\ 272 & 0 & 0.0 & \text { CHIEF SCIENTST }\end{array}$

295200.0 CHIEF SCIENTST

27200.0 DAFE CURATOR

295200.0 DAFE CURATOR

27200.0 GEOLOGIST

27200.0 GEOLOGIST

$\begin{array}{llll}272 & 0 & 0.0 & \text { GEOLOGIST } \\ 272 & 0 & 0.0 & \text { GEOLOGIST }\end{array}$

$\begin{array}{llll}272 & 0 & 0.0 & \text { GEOLOGIS.T }\end{array}$

295200.0 GEOLOGIST

295200.0 GEOLOGIST

$\begin{array}{llll}295 & 20 & 0.0 & \text { GEOLOGIST } \\ 295 & 20 & 0.0 & \text { GEOLOGIST }\end{array}$

27200.0 GEOPHYSICIST

295200.0 GEOPHYSICIST

27200.0 ELECTRONICS T

295200.0 ELECTRONICS T

$\begin{array}{llll}272 & 0 & 0.0 & \text { WATCH STANDER } \\ 272 & 0 & 0.0 & \text { WATCH STANDER }\end{array}$

272 OATCH STAMDER

27200.0 WATCH STANDER

27200.0 WATCH STANDER

27200.0 WATCH STANDER

295200.0 WATCH STANDER

295200.0 WATCH STANDER

295200 WATCH SIANDER

295200.0 WATCH STANDER

295200.0 WATCH STANDER

295200.0 WATCH STANDER

29520 .0 WATCH STANDER

27200.0 NAVIGATOR

272 O O.0 NAVIGATOR

295200.0 NAVIGATOR

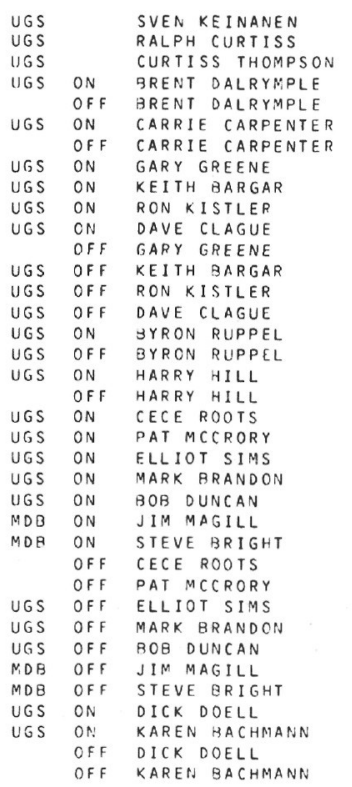

EQUIPMENT LIST
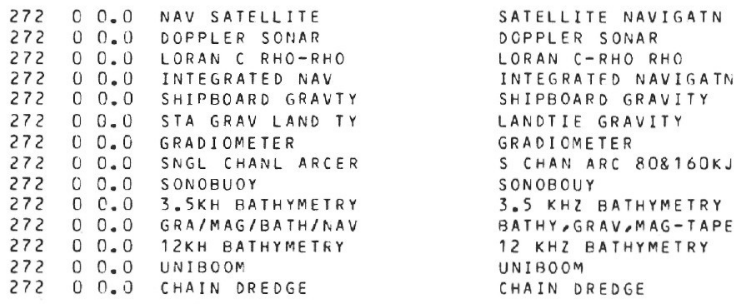

CHAIN OREDGE

INTEGRATED NAV OPRTING PERIODS

$\begin{array}{llllllll}273 & 20.0 & \text { SYSTEM } & G & \text { ON } & \text { LH } & & \text { STN/SPH } \\ 281 & 2134.0 & \text { SYSTEM } & G & \text { OFF } & \text { LH } & 20 & \text { STN/SPH } \\ 281 & 2236.0 & \text { SYSTEM } & G & \text { ON } & \text { LH } & 20 & \text { STN/SPH }\end{array}$

$5151.26-176 \quad 39.12$

$\begin{array}{rrrr}39 & 2.83 & 170 & 51.97 \\ 38 & 56.51 & 170 & 59.51\end{array}$

INTEGRATED NAV PRINTK LISTINGS

\begin{tabular}{|c|c|c|c|c|c|c|c|c|c|c|c|}
\hline 273 & 20.0 & LIST & 1 & START & L\# & & STN/SPA & 51 & 51.26 & -176 & 39.12 \\
\hline 2762 & 2248.0 & LIST & 1 & END & L\# & 001 & STN/SP\# & 49 & 19.03 & 175 & 3.22 \\
\hline 2762 & 2254.0 & L IST & 2 & START & L\# & 001 & STN/SP\# & 49 & 19.17 & 175 & \\
\hline 2812 & 2134.0 & LIST & 2 & END & L\# & 20 & STN/SPH & 39 & 2.83 & 170 & 51.97 \\
\hline 2812 & 2236.0 & LIST & 3 & START & L\# & 20 & STN/SPH & 38 & 56.51 & 170 & 59.51 \\
\hline 2861 & 1956.0 & LIST & 3 & END & LA & & STN/SPH & 28 & 11.55 & -177 & 21.03 \\
\hline 2882 & 208.3 & LIST & 4 & START & LA & & SIN/SPA & & & & \\
\hline 2952 & 202.0 & LIST & 4 & END & L\# & & STN/SPH & & & & \\
\hline
\end{tabular}


TABLE 2 - Continued

\begin{tabular}{|c|c|c|c|c|c|c|c|c|c|c|c|c|}
\hline \multirow{3}{*}{$\begin{array}{l}\text { JUL. } \\
\text { DAY }\end{array}$} & \multirow{3}{*}{$\begin{array}{l}\text { TIME } \\
\text { (GMT) }\end{array}$} & \multicolumn{2}{|c|}{ CRUISE/DATA INFO } & DATA & \multicolumn{3}{|c|}{ PERSONNEL,PORTS, EQUIPMENT } & \multirow{2}{*}{$\begin{array}{l}\text { WATER } \\
\text { DEPTH }\end{array}$} & \multirow{2}{*}{\multicolumn{2}{|c|}{ LATITUDE }} & \multirow{2}{*}{\multicolumn{2}{|c|}{ LONGITUDE }} \\
\hline & & RECORD. & SEQNCE & STATUSI & DES & RIPT & ION OR: & & & & & \\
\hline & & MEDIUM & NUMBER & INSTITUTE & & & STA./SHOT PT.\# & UNCOR. & DEG & MIN & DEG & MIN \\
\hline & & DR NAV & PLOT/LAB & MAP C & $O R P$ & OTS & & & & & & \\
\hline 273 & 70.0 & MAP/PLOT & 1 & START & LH & 1A & STN/SPH & & & & & \\
\hline 277 & 210.0 & MAP/PLOT & 2 & START & L\# & $1 \mathrm{E}$ & $\mathrm{STN} / \mathrm{SP} \#$ & & & & & \\
\hline 277 & 2330.0 & MAP/PLOT & 1 & END & L\# & $1 \mathrm{E}$ & STN/SP\# & & & & & \\
\hline 279 & 548.0 & MAP/PLOT & 2 & END & L\# & 2 & STN/SP\# & & & & & \\
\hline 279 & 548.2 & MAP/PLOT & 3 & START & LH & ? & STN/SPH & & & & & \\
\hline 279 & 120.0 & MAPIPLOT & 4 & START & L\# & 14 & STN/SPH & & & & & \\
\hline 279 & 1346.0 & MAP/PLOT & 3 & END & L\# & 14 & STN/SPH & & & & & \\
\hline 280 & 2230.0 & MAP/PLOT & 5 & START & L\# & 18 & STN/SP\# & & & & & \\
\hline 281 & 216.0 & MAP/PLOT & 4 & END & L\# & 18 & STN/SPH & & & & & \\
\hline 282 & 754.0 & MAP/PLOT & 5 & END & $L \#$ & 21 & STN/SPH & & & & & \\
\hline 282 & 830.0 & MAP /FLOT & 6 & START & L\# & 21 & STN/SPH & & & & & \\
\hline 283 & 742.0 & MAP/PLOT & 7 & START & $L \#$ & 23 & STN/SP\# & & & & & \\
\hline 283 & 752.0 & MAP/PLOT & 6 & END & $L \#$ & 23 & STN/SP\# & & & & & \\
\hline 284 & 10.0 & MAP / PLOT & $?$ & END & $L H$ & 23 & STN/SPH & & & & & \\
\hline 284 & 130.0 & MAP/PLOT & 8 & START & $L \#$ & 23 & STN/SPH & & & & & \\
\hline 285 & 832.0 & MAP/PLOT & 8 & END & $L \#$ & 24 & STN/SP\# & & & & & \\
\hline $\begin{array}{l}285 \\
286\end{array}$ & $\begin{array}{r}848.0 \\
1830.0\end{array}$ & $\begin{array}{l}\text { MAP/PLOT } \\
\text { MAP/PLOT }\end{array}$ & 9 & $\begin{array}{l}\text { START } \\
\text { END }\end{array}$ & $\begin{array}{l}\text { LH } \\
\text { LH }\end{array}$ & $\begin{array}{l}24 \\
27\end{array}$ & $\begin{array}{l}\text { STN/SPH } \\
\text { STN/SPA }\end{array}$ & & & & & \\
\hline 290 & 214.0 & MAP/PLOT & 10 & START & LA & & STN/SP\# & & & & & \\
\hline 291 & 1734.0 & MAP/PLOT & 10 & END & L\# & $3 ?$ & $\mathrm{SIN} / \mathrm{SP \#}$ & & & & & \\
\hline 291 & 1734.2 & MAPIPLOT & 11 & START & L $\#$ & & STN/SP\# & & & & & \\
\hline 291 & 1922.0 & MAP/PLOT & 11 & END & L $H$ & & STN/SPH & & & & & \\
\hline 291 & 2020.0 & MAP/PLOT & 12 & START & L\# & & STN/SP\# & & & & & \\
\hline 293 & 530.0 & MAP/PLOT & 12 & END & LH & 34 & STN/SPH & & & & & \\
\hline 293 & 530.2 & MAP/PLOT & 13 & START & LH & 35 & SIN/SP\# & & & & & \\
\hline 294 & 2126.0 & MAP/PLOT & 13 & END & $\mathrm{L} \#$ & 40 & SIN/SPH & & & & & \\
\hline 294 & 2150.0 & MAP/PLOT & 14 & SIART & LA & 40 & STN/SPH & & & & & \\
\hline 295 & 170.0 & MAP/PLOT & 14 & END & $L *$ & & STN/SPH & & & & & \\
\hline
\end{tabular}

TRACKLINES

\begin{tabular}{|c|c|c|c|c|c|c|c|c|c|c|c|}
\hline 273 & 741.0 & LINE & $1 \mathrm{~A}$ & START & L\# & $1 \mathrm{~A}$ & SIN/SPH & 51 & 39.17 & -177 & 6.86 \\
\hline 273 & 1536.0 & LINE & $1 \mathrm{~A}$ & END & LH & $1 \mathrm{~A}$ & STN/SPH & 51 & 9.01 & -179 & 3.80 \\
\hline 273 & 1537.0 & LINE & $1 B$ & START & LA & $1 B$ & STN/SPH & 51 & 8.95 & -179 & 4.06 \\
\hline 273 & 2137.0 & LINE & 18 & END & L* & 18 & STN/SPH & 50 & 48.17 & 179 & 28.39 \\
\hline 273 & 2138.0 & LINE & $1 \mathrm{c}$ & START & L\# & $1 C$ & STN/SP\# & 50 & 48.11 & 179 & 28.18 \\
\hline 274 & 955.0 & LINE & $1 \mathrm{c}$ & END & LH & $1 \mathrm{C}$ & STN/SPH & 50 & 12.34 & 176 & 59.54 \\
\hline 274 & 957.0 & LINE & 10 & START & LH & 10 & STN/SPH & 50 & 12.27 & 176 & 59.16 \\
\hline 278 & 032.0 & LINE & 10 & END & LH & 10 & STN/SPH & 49 & 45.67 & 173 & 27.81 \\
\hline 278 & 10.0 & LINE & IE & START & LH & TE & STN/SPH & 49 & 46.76 & 173 & 25.20 \\
\hline 278 & 170.0 & LINE & $1 \mathrm{E}$ & END & LH & $1 \mathrm{E}$ & STN/SPH & 47 & 1.75 & 172 & 30.72 \\
\hline 278 & 173.0 & LINE & 2 & START & L\# & 2 & STN/SPH & 47 & 1.27 & 172 & 30.53 \\
\hline 279 & 120.0 & LINE & 2 & END & L\# & 2 & STN/SPA & 45 & 11.32 & 169 & 37.29 \\
\hline 279 & 1210.0 & LINE & 14 & START & L\# & 14 & STN/SPA & 45 & 10.75 & 169 & 37.21 \\
\hline 280 & 318.0 & LINE & 14 & END & L\# & 14 & STN/SPH & 43 & 50.06 & 170 & 22.70 \\
\hline 280 & 320.0 & LINE & 15 & START & L\# & 15 & STN/SPH & 43 & 49.76 & 170 & 22.73 \\
\hline 280 & 730.0 & LINE & 15 & END & L\# & 15 & STN/SPH & 43 & 8.53 & 170 & 20.33 \\
\hline 280 & 738.0 & LINE & 16 & START & $L \#$ & 16 & $S T N / S P H$ & 43 & 7.37 & 170 & 20.12 \\
\hline 280 & 1143.0 & LINE & 16 & END & $L \#$ & 16 & STN/SPH & 42 & 35.71 & 170 & 44.91 \\
\hline 280 & $12 \quad 0.0$ & LINE & 17 & START & LH & 17 & STN/SPH & 42 & 33.63 & 170 & 44.38 \\
\hline 280 & 1748.0 & LINE & 17 & END & L\# & 17 & STN/SPH & 41 & 53.49 & 169 & 54.12 \\
\hline 280 & 1756.0 & LINE & 18 & START & L\# & 18 & $\mathrm{STN} / \mathrm{SPH}$ & 41 & 52.29 & 169 & 54.55 \\
\hline 281 & 630.0 & L INE & 18 & END & L\# & 18 & STN/SPH & 40 & 19.25 & 171 & 3.57 \\
\hline 281 & 632.0 & LINE & 19 & START & L\# & 19 & STN/SPH & 40 & 18.94 & 171 & 3.67 \\
\hline 281 & 1745.0 & LINE & 19 & ENO & LH & 19 & STN/SPH & 39 & 31.98 & 170 & 24.69 \\
\hline 281 & 1746.0 & LINE & 20 & START & L\# & 20 & STN/SPH & 39 & 31.86 & 170 & 24.64 \\
\hline 282 & 214.0 & LINE & 20 & END & $L \#$ & 20 & STN/SPH & 38 & 25.93 & 171 & 23.93 \\
\hline 282 & 219.0 & LINE & 21 & START & L\# & 21 & STN/SPA & 38 & 25.04 & 171 & 23.33 \\
\hline 282 & 120.0 & LINE & 21 & END & LH & 21 & STN/SPA & 37 & 40.77 & 169 & 52.90 \\
\hline 282 & $12 \quad 6.0$ & LINE & 22 & START & L\# & $2 ?$ & STN/SPH & 37 & 40.16 & 169 & 52.57 \\
\hline 283 & 56.0 & LINE & 22 & END & LH & 22 & STN/SPH & 35 & 12.72 & 171 & 43.53 \\
\hline 283 & 537.0 & LINE & 23 & START & L\# & 23 & STN/SPH & 35 & 10.05 & 171 & 48.13 \\
\hline 285 & 45.0 & LINE & 23 & END & L\# & 23 & STN/SP\# & 30 & 37.39 & 179 & 2.72 \\
\hline 285 & 419.0 & LINE & 24 & START & L* & 24 & STN/SPH & 30 & 35.98 & 179 & 3.80 \\
\hline 285 & 1122.0 & LINE & 24 & END & $L \#$ & 24 & STN/SPH & 29 & 37.36 & 179 & 4.04 \\
\hline 285 & 1127.0 & LINE & 25 & START & L\# & 25 & STN/SPH & 29 & 36.80 & 179 & 4.27 \\
\hline 285 & 220.0 & LINE & 25 & END & L\# & 25 & STN/SPH & 28 & 52.57 & -179 & 35.01 \\
\hline 285 & 222.0 & LINE & 26 & START & L\# & 26 & STN/SPH & 28 & 52.42 & -179 & 34.72 \\
\hline 286 & 1430.0 & LINE & 26 & END & LA & 25 & STN/SPH & 27 & 50.20 & -177 & 43.68 \\
\hline 286 & 1432.0 & LINE & 27 & START & L* & 27 & STN/SPH & 27 & 50.32 & -177 & 43.57 \\
\hline 286 & 1830.0 & LINE & 27 & ENO & L\# & 27 & STN/SPH & 28 & 7.45 & -177 & 26.54 \\
\hline 290 & 32.0 & LINE & 28 & START & LH & 23 & STN/SP\# & 28 & 5.18 & -177 & 18.26 \\
\hline 290 & 1030.0 & LINE & 28 & END & LA & 28 & STN/SPH & 26 & 56.86 & -176 & 30.79 \\
\hline 290 & 1032.0 & LINE & 29 & START & $L \#$ & 29 & STN/SPH & 26 & 56.80 & -176 & 30.44 \\
\hline 291 & 049.0 & LINE & 29 & END & LH & 29 & STN/SPH & 26 & 27.57 & -973 & 53.85 \\
\hline 291 & 050.0 & LINE & 30 & START & $L \#$ & 30 & $\mathrm{STN} / \mathrm{SPH}$ & 26 & 27.50 & -173 & 53.69 \\
\hline 291 & 428.0 & LINE & 30 & END & $L \#$ & 30 & STN/SPH & 26 & 2.79 & -173 & 23.41 \\
\hline 291 & 430.0 & LINE & 31 & START & $L \#$ & 31 & STN/SPH & 26 & 2.65 & -173 & 23.07 \\
\hline 291 & 130.0 & LINE & 31 & END & L\# & 39 & STN/SPH & 25 & 32.87 & -171 & 55.98 \\
\hline 291 & 133.0 & LINE & 32 & START & $L H$ & 32 & STN/SPH & 25 & 32.99 & -171 & 55.01 \\
\hline
\end{tabular}


TABLE 2 - Continued

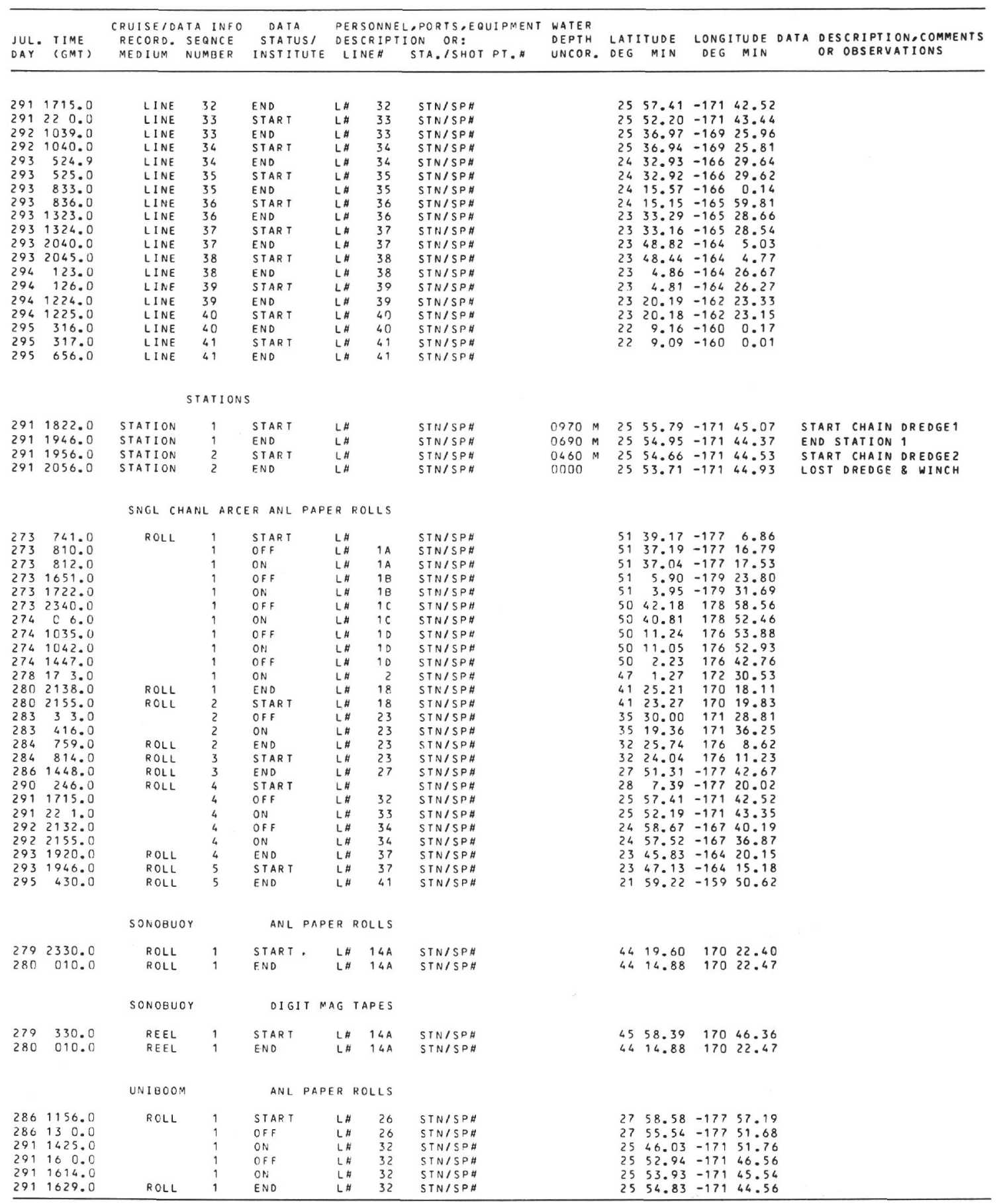


TABLE 2 - Continued

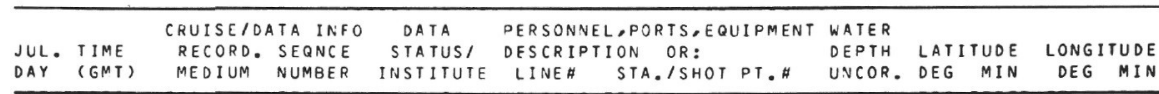

3.5KH BATHYMETRY ANL PAPER ROLLS

$\begin{array}{rr}273 & 741.0 \\ 273 & 90.0 \\ 273 & 945.0 \\ 273 & 210.0 \\ 273 & 228.0 \\ 274 & 1443.0 \\ 278 & 50.0 \\ 279 & 900.0 \\ 279 & 920 \\ 281 & 1456.0 \\ 281 & 1746.0 \\ 286 & 1830.0 \\ 290 & 246.0 \\ 292 & 542.0 \\ 292 & 630.0 \\ 295 & 547.0 \\ 295 & 616.0 \\ 295 & 717.0\end{array}$

$\begin{array}{lll}\text { ROLL } & 1 & \text { START } \\ & 1 & \text { OFF } \\ & 1 & \text { ON } \\ & 1 & \text { OFF } \\ \text { ROLL } & 1 & \text { ON } \\ \text { ROLL } & 1 & \text { END } \\ & 2 & \text { START } \\ & 2 & \text { OFF } \\ & 2 & \text { ON } \\ & 2 & \text { OFF } \\ \text { ROLL } & 2 & \text { ON } \\ \text { ROLL } & 2 & \text { END } \\ & 3 & \text { START } \\ & 3 & \text { OFF } \\ & 3 & \text { ON } \\ & 3 & \text { OFF } \\ & 3 & \text { ON } \\ & 3 & \text { END }\end{array}$

$L \#$
$L \#$
$L \#$
$L \#$
$L \#$
$L \#$
$L \#$
$L \#$
$L \#$
$L \#$
$L \#$
$L \#$
$L \#$
$L \#$
$L \#$
$L \#$
$L \#$
$L \#$
$L \#$
$L \#$
$L \#$
$L \#$

STN/SPH
STN/SPH
STN/SPH
STN/SPH
STN/SPH
STN/SPH
STN/SPH
STN/SPH
STN/SPH
STN/SPH
STN/SPH
STN/SPH
STN/SPH
STN/SPH
STN/SPH
STN/SPH
STN/SPH
STN/SPH

12KH BATHYMETRY ANL PAPER ROLLS

$\begin{array}{rr}273 & 741.0 \\ 273 & 2037.7\end{array}$$$
\text { ROL }
$$$$
\text { ROLL }
$$$$
\begin{aligned}
& \text { START } \\
& \text { END }
\end{aligned}
$$$$
\text { L\# } 1 \mathrm{~A}
$$

STN / SPA

SHIPBOARD MAGGY OPRTING PERIODS

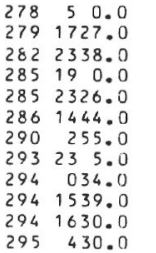

SYSTEM

SYSTEM

SYSTEM

SYSTEM

SYSTEM

SYSTEM

SYSTEM

SYSTEM

SYSTEM

$\begin{array}{lll}\text { ON } & \text { LH } & 1 E \\ \text { OFF } & \text { LH } & 14 \\ \text { ON } & \text { LH } & 22 \\ \text { OFF } & \text { LH } & 25 \\ \text { ON } & \text { LH } & 26 \\ \text { OFF } & \text { LH } & 27 \\ \text { ON } & \text { LH } & \\ \text { OFF } & \text { LH } & 38 \\ \text { ON } & \text { LH } & 38 \\ \text { OFF } & \text { LH } & 40 \\ \text { ON } & \text { LH } & 40 \\ \text { OFF } & \text { LH } & 49\end{array}$

STN/SPH

STN/SPH

STN/SPH
STN/SPH

STN STSPE

STN/SPA

STN/SPH

STN/SPH

STN/SPA

STN/SPA

STN/SPH
SHIPBOARD MAGGY ANL PAPER ROLLS

\begin{tabular}{|c|c|c|c|c|c|c|c|}
\hline 278 & 50.0 & ROLL & 3 & START & L\# & $1 E$ & SIN/SPH \\
\hline 2791 & 120.0 & ROLL & 3 & END & LA & 2 & SIN/SPH \\
\hline 2791 & 128.0 & ROLL & 4 & START & LH & 014 & STN/SPH \\
\hline $279 \quad 1$ & 1727.0 & & 4 & OF F & L\# & 14 & STN/SPH \\
\hline 2822 & 2338.0 & & 4 & ON & LH & 022 & STN/SPH \\
\hline 284 & 530.0 & ROLL & 4 & END & LH & 23 & STN/SPH \\
\hline 284 & 540.0 & ROLL & 5 & STAR T & LH & 23 & SIN/SPH \\
\hline 2851 & 1960.0 & ROLL & 5 & END & $L \#$ & 25 & STN/SP\# \\
\hline 2851 & 1950.0 & ROLL & 6 & START & LH & 25 & STN/SPH \\
\hline 2861 & 1444.0 & & 6 & OFF & LH & 27 & STN/SP\# \\
\hline 290 & 255.0 & & 6 & ON & LA & 28 & SIN/SPH \\
\hline 2902 & $22 \quad 2.0$ & ROLL & 0 & END & L\# & 29 & STN/SPH \\
\hline 2902 & 227.0 & ROLL & 7 & START & L\# & 29 & SIN/SPH \\
\hline 2911 & 1430.0 & & 7 & $O F F$ & L\# & 32 & STN/SPH \\
\hline 2912 & 220.0 & & 7 & ON & $L \#$ & 33 & STN/SPH \\
\hline 2921 & 1750.0 & & 7 & $O F F$ & L\# & 34 & STN/SPH \\
\hline 2922 & 215.0 & & 7 & ON & L\# & 34 & STN/SPA \\
\hline 2922 & 232.0 & ROLL & 7 & END & L\# & 34 & STN/SPH \\
\hline 2922 & 236.0 & ROLL & 8 & STAR T & L\# & 34 & STN/SPA \\
\hline 2932 & 235.0 & & 8 & $O F F$ & $L \#$ & 38 & STN/SPA \\
\hline 294 & 034.0 & & 8 & $O N$ & L\# & 38 & STN/SPH \\
\hline 2941 & 1130.0 & ROLL & 8 & END & L\# & 39 & STN/SPH \\
\hline 2949 & 1137.0 & ROLL & 9 & START & L\# & 39 & STN/SPA \\
\hline 2941 & 1539.0 & & 9 & OF $\mathrm{F}$ & $\mathrm{L} \#$ & 40 & $\mathrm{STN} / \mathrm{SPA}$ \\
\hline 941 & 1630.0 & & 9 & ON & L\# & 40 & STN/SPA \\
\hline & & & & & & & \\
\hline
\end{tabular}

GRADIOMETER OPRTING PERIODS

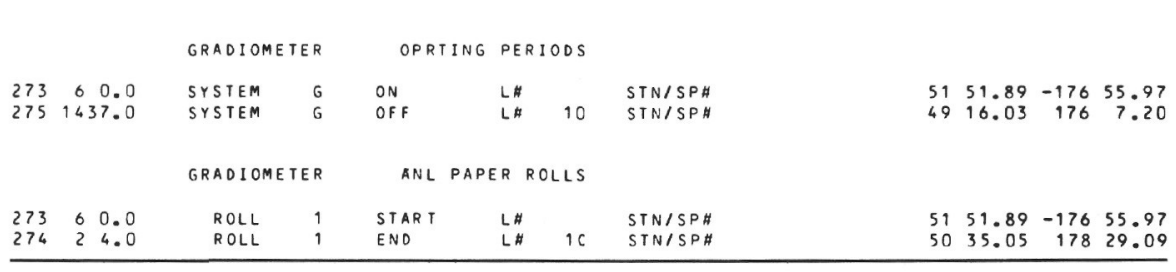
$23 \quad 6.95-16150.11$ $\begin{array}{llll}26 & 34.21 & -174 & 22.89\end{array}$ $\begin{array}{llll}51 & 39.17 & -177 & 6.86\end{array}$

$\begin{array}{llll}51 & 34.33 & -177 & 27.18 \\ 51 & 32.03 & -177 & 36.43\end{array}$

$\begin{array}{llll}51 & 34.03 & -177 & 27.18 \\ 50 & 50.53 & -179 & 36.43\end{array}$

$\begin{array}{llll}50 & 50.52 & 179 & 36.37 \\ 50 & 46.47 & 179 & 20.87\end{array}$

$\begin{array}{llll}50 & 46.47 & 179 & 20.87 \\ 50 & 2.39 & 176 & 42.88\end{array}$

$\begin{array}{llll}49 & 8.61 & 173 & 10.41\end{array}$

$\begin{array}{llll}45 & 27.02 & 169 & 57.53\end{array}$

$\begin{array}{llll}45 & 26.82 & 169 & 57.36\end{array}$

$\begin{array}{llll}39 & 41.69 & 170 & 42.33\end{array}$

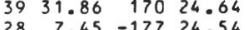

$\begin{array}{llll}28 & 7.39 & -177 & 20.02\end{array}$

$2543.59-17019.57$

$\begin{array}{lllll}51 & 39.17 & -177 & 6.86\end{array}$

$\begin{array}{llll}44 & 43.71 & 173 & 10.41\end{array}$

$\begin{array}{llll}44 & 43.72 & 170 & 6.40 \\ 35 & 58.53 & 171 & 9.56\end{array}$

$\begin{array}{rrrr}35 & 58.53 & 171 & 9.56 \\ 29 & 5.96 & -180 & 0.33\end{array}$ $\begin{array}{llll}29 & 5.96 & -180 & 0.33 \\ 28 & 45.92 & -179 & 23.60\end{array}$ $\begin{array}{llll}27 & 59.06 & -177 & 42.90\end{array}$ $\begin{array}{llll}23 & 26.20 & -164 & 16.47\end{array}$ $2311.45-16424.16$ $\begin{array}{rrrr}23 & 2.98 & -161 & 41.76 \\ 21 & 59.22 & -159 & 50.62\end{array}$

$\begin{array}{lrll}49 & 8.61 & 173 & 10.41\end{array}$ $\begin{array}{llll}45 & 11.32 & 169 & 37.29\end{array}$ $\begin{array}{llll}45 & 10.86 & 169 & 37.22 \\ 44 & 43.72 & 170 & 6.40\end{array}$ $\begin{array}{llll}44 & 43.72 & 170 & 6.40 \\ 35 & 58.53 & 171 & 9.56\end{array}$ $\begin{array}{llll}32 & 39.75 & 175 & 44.67\end{array}$ $\begin{array}{rrrr}32 & 38.66 & 175 & 46.03 \\ 29 & 2.58 & -179 & 54.85\end{array}$ $29 \quad 1.70-179 \quad 53.52$ $2751.06-17742.90$ $\begin{array}{lllll}28 & 6.15 & -177 & 19.03\end{array}$ $26 \quad 34.34-174 \quad 23.52$ $\begin{array}{llll}25 & 46.72 & -179 & 51.50 \\ 25 & 52.20 & -179 & 43.44\end{array}$ $2519.00-168 \quad 13.31$ $25 \quad 0.18-167 \quad 44.36$ $\begin{array}{llll}24 & 54.12 & -167 & 27.39\end{array}$ $\begin{array}{lllll}24 & 53.94 & -167 & 26.82\end{array}$ $\begin{array}{llll}23 & 26.20 & -164 & 16.47\end{array}$ $\begin{array}{lllll}23 & 11.45 & -164 & 24.16\end{array}$ $\begin{array}{lllll}23 & 18.69 & -162 & 33.38\end{array}$ $\begin{array}{llll}23 & 18.93 & -162 & 32.09\end{array}$ $\begin{array}{llll}23 & 6.95 & -161 & 50.11\end{array}$ $\begin{array}{rrrr}23 & 2.98 & -161 & 41.76 \\ 21 & 59.22 & -159 & 50.62\end{array}$ 
TABLE 2 - Continued

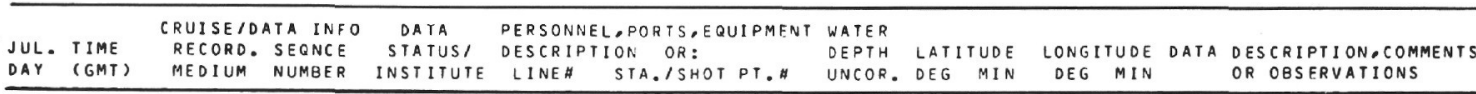

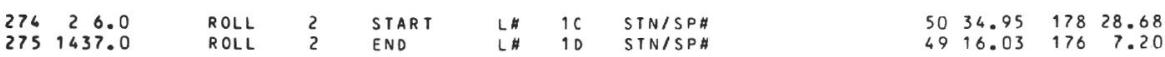

SHIPBOARD GRAVTY OPRTING PERIODS

\begin{tabular}{|c|c|c|c|c|c|c|c|c|c|c|c|}
\hline 272 & 530.0 & SYSTEM & G & ON & $L \#$ & & STN/SPH & & & & \\
\hline 274 & 100.0 & SYSTEM & G & $O F F$ & $L \#$ & 10 & STN/SPH & 50 & 12.20 & 176 & 58.59 \\
\hline 278 & 548.0 & SYSTEM & $G$ & $O N$ & $L \#$ & $1 \mathrm{E}$ & STN/SPH & 49 & 3.51 & 173 & 12.00 \\
\hline 278 & 932.0 & SYSTEM & $G$ & OF F & L\# & $1 \mathrm{E}$ & STN/SP\# & 48 & 22.93 & 173 & 2.11 \\
\hline 278 & 1031.0 & SYSTEM & G & $O N$ & LA & $1 \mathrm{E}$ & SIN/SPH & 48 & 11.82 & 172 & 57.15 \\
\hline 279 & 2032.0 & SYSTEM & G & OFF & L\# & 14 & STN/SPH & 44 & 32.47 & 170 & 16.66 \\
\hline 28 & 930.0 & SYSTEM & G & $O N$ & L\# & 16 & STN/SPH & 42 & 53.00 & 170 & 32.05 \\
\hline 28 & 1055.0 & SYSTEM & G & $O F F$ & LH & 19 & $S T N / S P H$ & 39 & 54.77 & 171 & 0.96 \\
\hline 28 & 210.0 & SYSTEM & $\mathrm{G}$ & ON & $L \#$ & 20 & STN/SPH & 39 & 6.30 & 170 & 47.83 \\
\hline
\end{tabular}

SHIPBOARO GRAVTY ANL PAPER ROLLS

\begin{tabular}{|c|c|c|c|c|c|c|c|}
\hline 273 & 24.0 & ROLL & 1 & STAR T & L\# & & STN/SPH \\
\hline 274 & 724.0 & ROLL & 1 & & LA & IC & STN/SPH \\
\hline 274 & 733.0 & ROLL & 2 & START & L\# & $1 \mathrm{C}$ & SIN/SPH \\
\hline 274 & $10 \quad 0.0$ & & 2 & OFF & LA & 10 & STN/SP \\
\hline 278 & 548.0 & & 2 & $O N$ & LA & $1 E$ & STN/SPH \\
\hline 278 & 930.0 & & 2 & OF F & LH & IE & STN/SPH \\
\hline 278 & 1030.0 & & 2 & ON & LA & iE & STN/SP \\
\hline 279 & 120.0 & ROLL & 2 & END & L\# & 2 & STN/SP \\
\hline 279 & 1210.0 & ROLL & 3 & START & L\# & ? & SIN/SPH \\
\hline 279 & 2032.0 & & 3 & $O F F$ & L\# & 14 & $\mathrm{STN} / \mathrm{SP}$ \\
\hline 280 & 930.0 & & 3 & $O N$ & $\mathrm{~L} \#$ & 16 & STN/S \\
\hline 281 & 1055.0 & ROLL & 3 & END & L\# & 19 & STN/SP \\
\hline 281 & 210.0 & ROLL & 4 & START & L\# & 20 & STN/SPH \\
\hline 283 & 110.0 & ROLL & 4 & END & LA & 023 & STN/SPH \\
\hline 283 & 195.0 & ROLL & 5 & START & $L \#$ & 023 & STN/SPH \\
\hline 285 & 19.0 & ROLL & 5 & END & $L \#$ & 23 & $S T N / S P H$ \\
\hline 285 & 113.0 & ROLL & 6 & START & LA & 23 & STN/SF \\
\hline 286 & 1439.0 & ROLL & 6 & END & LA & 27 & $S T N / S P$ \\
\hline 286 & 1442.0 & ROLL & 7 & START & L\# & 27 & STN/SF \\
\hline 286 & 1830.0 & & 7 & OF F & LH & 27 & STN/SPH \\
\hline 290 & 255.0 & & 7 & $O N$ & $L \#$ & 28 & STN/SPA \\
\hline 291 & 107.0 & ROLL & 7 & END & L\# & 31 & STN/SPH \\
\hline 291 & 1011.0 & ROLL & 8 & STAR T & L\# & 31 & STN/SF \\
\hline 291 & 1730.0 & & 8 & $O F F$ & $L \#$ & 32 & STN/SPA \\
\hline 291 & 220.0 & & 8 & ON & $L \#$ & 33 & STN/SP\# \\
\hline 292 & 2338.0 & ROLL & 8 & END & L\# & 34 & STN/SPA \\
\hline 292 & 2342.0 & ROLL & 9 & STAR T & L\# & 36 & STN/S \\
\hline 294 & 1140.0 & ROLL & 9 & END & LH & 39 & $\mathrm{STN} / \mathrm{SP}$ \\
\hline 294 & 1143.0 & ROLL & 10 & START & L\# & 39 & STN/SPH \\
\hline 642 & 193 & ROLL & 10 & END & L\# & & STN/SPH \\
\hline
\end{tabular}

$51 \quad 51.27 \quad-176 \quad 39.13$

$5018.70 \quad 177 \quad 26.75$

50 $12.20 \quad 176 \quad 58.59$

$49 \quad 3.51 \quad 173 \quad 12.00$

$\begin{array}{llll}48 & 23.29 & 173 & 2.28\end{array}$

$\begin{array}{llll}48 & 11.98 & 172 & 57.22\end{array}$

$\begin{array}{llll}45 & 11.32 & 169 & 37.29\end{array}$

$\begin{array}{llll}45 & 10.75 & 169 & 37.21\end{array}$

$\begin{array}{lllll}44 & 32.47 & 170 & 16.60\end{array}$

$\begin{array}{llll}42 & 53.00 & 170 & 32.05 \\ 39 & 54.77 & 171 & 0.96\end{array}$

$\begin{array}{rrrr}39 & 54.77 & 171 & 0.96 \\ 39 & 6.30 & 170 & 47.83\end{array}$

$\begin{array}{llll}34 & 35.49 & 172 & 49.83\end{array}$

$\begin{array}{llll}34 & 34.93 & 172 & 42.20\end{array}$

$30 \quad 50.39 \quad 178 \quad 40.33$

$3050.12 \quad 178 \quad 40.86$

$\begin{array}{llll}30 & 50.12 & 178 & 40.83\end{array}$

$2750.75-17743.18$

$2850.94-17743.01$

$280.45-177 \quad 19.03$

$\begin{array}{llll}25 & 42.67 & -172 & 25.39\end{array}$

$2542.37-17224.75$

$\begin{array}{llll}25 & 42.37 & -172 & 24.75 \\ 25 & 58.15 & -171 & 42.08\end{array}$

$25 \quad 52.20-17143.44$

$2452.45-967 \quad 22.27$

$\begin{array}{lllll}24 & 52.27 & -167 & 21.71\end{array}$

$\begin{array}{llll}23 & 19.03 & -162 & 31.53 \\ 23 & 19.12 & -162 & 30.97\end{array}$

GRA/MAG/BATH/NAV DIGIT MAG TAPES

$273 \quad 6 \quad 0.0$

$274 \quad 100.0$

275045.0

REEL 1 START

REEL 1 ENO

REEL 2 START

$\begin{array}{lll}\text { REEL } & 2 & \text { END } \\ \text { REEL } & 3 & \text { START }\end{array}$

START

280195.0

2801920 .

289040.0

281055.0

$\begin{array}{ll}282 & 4 \\ 282 & 545\end{array}$

$\begin{array}{ll}282 & 545.0\end{array}$

$\begin{array}{ll}285 & 2320.0 \\ 285 & 2326.0\end{array}$

2861830.0

$290 \quad 255.0$

290
290
251.0

$290 \quad 446.0$

$\begin{array}{rrr}290 & 11 & 0.0 \\ 292 & 630.0\end{array}$

292630.0

$\begin{array}{rr}292 & 752.0 \\ 292 & 1750.0\end{array}$

292215.0

2931812.0

2931824.0

2931830.0

2932030.0

2932117.0

2941318.0

2941324.0

2941814.0

294
2952057.0

$\begin{array}{lll} & 3 & \text { ON } \\ \text { REEL } & 3 & \text { END }\end{array}$

END

REEL

REEL

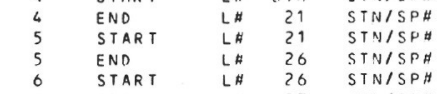

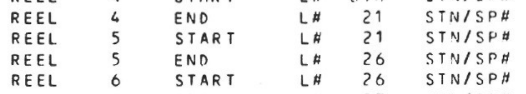

SIN/SPH
STN/SPH

STN/SPH
STN/SPH

STN/SPA

STNISPA

STNISPA

STN/SPH

STN/SPH

STN/SP

STN/SP

STN/SPA

STN/SPA

STN/SP

STN/SPA

STN/SPA

STN/SPa

STN SPP

STH/SP

STN/SPA

STN/SPA

STN/SPA

STN/SPA

STN/SPA

STN/SPH

STN/SPA

STN/SPH

STN/SPA

STN/SPA

STN/SPA

REEL

ON

STN/SP

$\begin{array}{llll}51 & 51.89 & -176 & 55.97\end{array}$

$\begin{array}{lllll}50 & 12.20 & 176 & 58.59\end{array}$

$\begin{array}{lllll}48 & 34.72 & 176 & 21.10\end{array}$

$4945.03 \quad 17324.06$

$\begin{array}{llll}49 & 39.84 & 173 & 20.64\end{array}$

$\begin{array}{llll}41 & 43.32 & 170 & 1.09\end{array}$

$\begin{array}{lllll}41 & 41.37 & 170 & 2.45\end{array}$

$\begin{array}{llll}41 & 1.87 & 170 & 35.30\end{array}$

40 $59.60 \quad 170 \quad 35.98$

$\begin{array}{llll}38 & 16.60 & 171 & 7.35\end{array}$

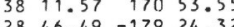

$2846.49-17924.32$

$2845.92-17923.60$

$280.45-17724.54$

$\begin{array}{llll}28 & 8.15 & -177 & 19.03\end{array}$

$2758.97-177-13.31$

$\begin{array}{llll}27 & 58.41 & -177 & 12.8\end{array}$

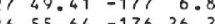

$\begin{array}{llll}26 & 55.64 & -176 & 26.22 \\ 25 & 42.11 & -170 & 10.46\end{array}$

$2540.45-16955.32$

$2511.00-168 \quad 13.31$

$25 \quad 0.18-16744.36$

$23 \quad 4 ? .86-164 \quad 33.14$

$23 \quad 43.40-164 \quad 30.88$

$2343.62-16429 . ? 1$

$\begin{array}{llll}23 & 48.56 & -164 & 6.89\end{array}$

$2342.07-164 \quad 8.07$

$2316.85-162 \quad 14.20$

$\begin{array}{llll}23 & 16.44 & -162 & 13.14\end{array}$

$\begin{array}{rrrr}22 & 53.12 & -169 & 25.06 \\ 22 & 44.65 & -161 & 8.42\end{array}$

STA GRAV LAND TY OPRTING PERIODS

\begin{tabular}{|c|c|c|c|c|c|c|c|c|c|c|c|c|c|}
\hline \multirow{2}{*}{$\begin{array}{l}295 \\
295\end{array}$} & $\begin{array}{l}0 \quad 0.0 \\
0\end{array}$ & SYSTEM & $G$ & ON & $L \#$ & STN/SPH & & & 22 & 25.42 & -160 & 30.68 & \\
\hline & & CHAIN & DREDGE & SAMPLF A & ATTEMPTS & & & & & & & & \\
\hline 291 & 1822.0 & SAMPLE & 1 & START & L\# & $S T N / S P H$ & 0970 & m & 25 & 55.79 & -171 & 45.07 & CHAIN BAG DREDGE \\
\hline 291 & 1946.0 & SAMPLE & 1 & END RECOV & $L \#$ & $S T N / S P \#$ & 0690 & M & 25 & 54.95 & -171 & 44.37 & CHAIN BAG DREDGE \\
\hline 291 & 1956.0 & SAMPLE & 2 & START & L\# & STN/SPH & 0460 & m & 25 & 54.66 & -171 & 44.53 & CHAIN BAG DREDGE \\
\hline 91 & 2042.0 & SAMPLE & 2 & END NO RE & L\# & SIN/SPA & 0000 & & 25 & 54.14 & -171 & 44.79 & LOST BAG \& 350 MWIRE \\
\hline
\end{tabular}

Note: Jackson/Dalrymple chief scientists. Listing generated by data type. 21 March 1978. 
PRE-LEG 55 SITE SURVEY GEOPHYSICAL DATA

TABLE 3

Data Summary for Cruise LEE8-76-NP

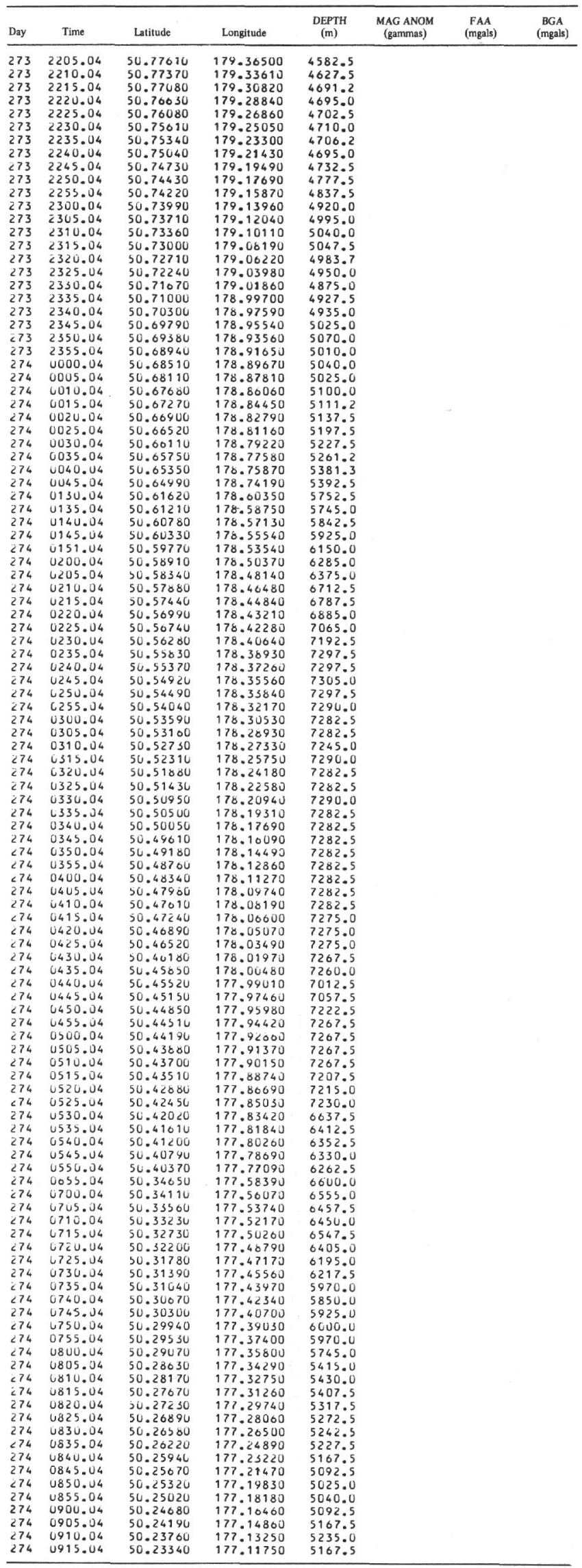

TABLE 3 - Continued

\begin{tabular}{|c|c|c|c|c|c|c|c|}
\hline Day & Time & Latitude & Longitude & $\begin{array}{l}\text { DEPTH } \\
\text { (m) }\end{array}$ & $\underset{\text { (gammas) }}{\text { MAG ANOM }}$ & $\underset{\text { (mgals) }}{\mathrm{FAA}}$ & $\begin{array}{c}\text { BGA } \\
\text { (mgals) }\end{array}$ \\
\hline 274 & 0920.04 & 50.23030 & 177.10090 & 5160.0 & & & \\
\hline 274 & 0925.04 & 50.22760 & 177.08500 & 5175.0 & & & \\
\hline 274 & 0930.04 & 50.22400 & 177.06940 & 5130.0 & & & \\
\hline 274 & 0935.04 & 50.22090 & 177.05280 & 5070.0 & & & \\
\hline 274 & 0940.04 & 50.21610 & 177.03850 & 5047.5 & & & \\
\hline 274 & 0945.04 & 56.21270 & 177.02260 & 5115.0 & & & \\
\hline 274 & 0950.04 & 50.20930 & 177.00750 & 5100.0 & & & \\
\hline 274 & 0955.04 & 50.20570 & 176.99230 & 5017.5 & & & \\
\hline 278 & US 00.04 & 49.14340 & 173.17350 & & -71.6 & & \\
\hline 278 & 0505.04 & 49.13600 & 173.17620 & & -98.1 & & \\
\hline 278 & 0510.04 & 49.12570 & 173.17900 & & -143.7 & & \\
\hline 278 & 0515.04 & 49.11090 & 173.18180 & & -198.2 & & \\
\hline 278 & 0520.04 & 49.10800 & 173.18450 & & -237.7 & & \\
\hline 278 & 0525.04 & 49.09420 & 173.18730 & & -272.2 & & \\
\hline 278 & 0530.04 & 49.09030 & 173.19010 & & -298.8 & & \\
\hline 278 & 0535.04 & 49.08150 & 173.19290 & 5220.0 & -319.3 & & \\
\hline 278 & 0540.04 & 49.07260 & 173.19560 & 5235.0 & -335.8 & & \\
\hline 278 & 0548.54 & 49.05700 & 173.20040 & 5188.5 & -330.3 & 29.2 & 386.5 \\
\hline 278 & 0553.54 & 49.04740 & 173.20320 & 5175.0 & -308.5 & 28.4 & 384.8 \\
\hline 278 & 0558.54 & 49.03400 & 173.20020 & & -278.9 & 40.1 & \\
\hline 278 & 0603.54 & 49.02140 & 173.20030 & 5044.5 & -257.7 & 12.3 & 359.7 \\
\hline 278 & 0008.54 & 49.00910 & 173.19070 & 4884.8 & -249.3 & 13.6 & 350.0 \\
\hline 278 & 0613.54 & 48.99680 & 173.18100 & 4790.3 & -252.9 & 14.5 & 344.4 \\
\hline 278 & 0018.54 & 48.98450 & 173.17140 & 4738.5 & -259.3 & 16.3 & 342.6 \\
\hline 278 & 0623.54 & 48.96950 & 173.16530 & 4751.2 & -253.1 & 44.5 & 371.7 \\
\hline 278 & 0028.54 & 48.94790 & 173.16780 & 4846.5 & -228.5 & 43.0 & 376.7 \\
\hline 278 & 0033.54 & 48.93620 & 173.16050 & 5003.2 & -195.4 & 28.3 & 372.8 \\
\hline 278 & 0038.54 & 48.91430 & 173.16360 & 5034.0 & -167.3 & 25.3 & 371.9 \\
\hline 278 & 0643.54 & 48.89834 & 173.16070 & 5061.7 & -157.2 & 21.7 & 370.3 \\
\hline 278 & 0648.54 & 48.88230 & 173.15780 & 5145.7 & -160.8 & 18.7 & 373.0 \\
\hline$<78$ & 0653.54 & 48.86030 & 173.15490 & 5311.5 & -168.8 & 13.2 & 378.9 \\
\hline 278 & 6058.54 & 48.84990 & 173.15190 & & -168.9 & 7.9 & \\
\hline 278 & C703.54 & 48.83890 & 173.14920 & 5542.5 & -160.1 & 8.1 & 389.8 \\
\hline 278 & 6708.54 & 48.83180 & 173.16710 & 5563.5 & -139.8 & 0.7 & 389.8 \\
\hline 278 & 0713.54 & 68.82460 & 173.16500 & 5588.2 & -116.4 & 6.4 & 391.2 \\
\hline 278 & 0718.54 & 48.81740 & 173.14290 & 5600.2 & -98.8 & 6.3 & 391.9 \\
\hline 278 & 6723.54 & $48.810<0$ & 173.16080 & 5597.3 & -98.3 & -14.7 & 370.7 \\
\hline 278 & 6728.54 & 48.80310 & 173.13870 & 5490.0 & -113.9 & 6.3 & 384.3 \\
\hline 278 & 0733.54 & 48.78726 & 173.13570 & 5969.5 & -138.3 & 18.0 & 373.4 \\
\hline 278 & 0738.54 & 48.70770 & 173.13240 & 4620.0 & -168.3 & 30.5 & 348.6 \\
\hline 278 & 0743.54 & 48.74810 & 173.12900 & 4172.3 & -194.1 & 42.2 & 329.5 \\
\hline 278 & 0748.54 & 48.72860 & 173.12570 & 3558.8 & -216.8 & 56.8 & 301.9 \\
\hline 278 & 0753.54 & 48.70900 & 173.12240 & 3156.0 & -226.0 & 71.2 & 288.5 \\
\hline 278 & 0758.54 & 48.68950 & 173.11900 & & -193.7 & 81.5 & \\
\hline 278 & 4863.54 & 48.67020 & 173.11500 & 2756.3 & -137.3 & 85.1 & 274.9 \\
\hline 278 & 0808.54 & 48.65110 & 173.11220 & 3231.7 & -105.8 & 81.7 & 304.2 \\
\hline 278 & 6813.54 & 48.63200 & 173.10880 & 3790.5 & -130.0 & 73.1 & 334.5 \\
\hline 278 & 0818.54 & 48.61290 & 173.10540 & 4128.7 & -190.9 & 63.2 & 347.5 \\
\hline 278 & 4823.54 & 48.54380 & 173.10190 & 4307.2 & -244.3 & 54.0 & 350.6 \\
\hline 278 & 0828.54 & 48.57470 & 173.09850 & 4505.2 & -257.5 & 46.2 & 360.6 \\
\hline 278 & 0833.54 & 48.55940 & 173.09400 & 4815.0 & -237.4 & 39.9 & 371.5 \\
\hline 278 & 0838.54 & 48.54560 & 173.09050 & 4887.7 & -200.8 & 37.5 & 374.1 \\
\hline 278 & 0843.54 & 48.53160 & 173.08630 & 4821.8 & -150.1 & 37.5 & 369.5 \\
\hline 278 & 4848.54 & 48.51590 & 173.08230 & 4593.0 & -111.9 & 39.0 & 355.3 \\
\hline 278 & 0853.54 & 48.50010 & 173.07840 & 4287.0 & -82.0 & 34.4 & 329.6 \\
\hline 278 & 0858.54 & 48.48440 & 173.07440 & & -65.4 & 45.3 & \\
\hline 278 & 0903.54 & 48.46800 & 173.00940 & 3900.0 & -56.4 & 11.9 & 280.5 \\
\hline 278 & 0908.54 & 48.45280 & 173.06390 & 4073.2 & -47.1 & 25.3 & 305.8 \\
\hline 278 & 0913.54 & 48.43700 & 173.05850 & 4373.2 & -32.1 & 31.7 & 332.8 \\
\hline$<78$ & 4918.54 & 48.42210 & 173.05230 & 4585.5 & -14.8 & 26.5 & 342.3 \\
\hline 278 & 0923.54 & 48.40740 & 173.04610 & 4934.2 & -4.3 & -14.0 & 325.8 \\
\hline 278 & 0928.54 & 48.39260 & 173.03980 & 5382.7 & 3.1 & 2.8 & 373.5 \\
\hline
\end{tabular}

$278 \quad 0940.04$

$278 \quad 0945.04$

278 0955.04

$\begin{array}{ll}278 & 1000.04 \\ 278 & 1005.04\end{array}$

$278 \quad 1010.04$

$\begin{array}{ll}278 & 1015.04 \\ 278 & 1020.04\end{array}$

$278 \quad 1033.54$

$278 \quad 1038.54$

$278 \quad 1043.54$

$\begin{array}{ll}278 & 1053.54 \\ 278 & 1058.54\end{array}$

$278 \quad 1103.54$

$278 \quad 1113.54$

$278 \quad 1116.54$

$278 \quad 1123.54$

$278 \quad 1133.54$

278 1138.54

$\begin{array}{ll}278 & 1148.54 \\ 278 & 1153.54\end{array}$

$\begin{array}{ll}278 & 1153.54 \\ 278 & 1158.54\end{array}$

$\begin{array}{ll}278 & 1203.54 \\ 278 & 1208.54\end{array}$

$278 \quad 1213.54$

$\begin{array}{ll}278 & 1218.54 \\ 278 & 1223.54\end{array}$

$\begin{array}{ll}278 & 1228.54 \\ 278 & 1233.54\end{array}$

$278 \quad 1238.54$

$278 \quad 1243.54$

$278 \quad 1253.54$

$\begin{array}{ll}278 & 1258.54 \\ 278 & 1303.54\end{array}$

$\begin{array}{ll}278 & 1308.54 \\ 278 & 1313.54\end{array}$

$\begin{array}{ll}278 & 1318.54 \\ 278 & 1323.54\end{array}$

$\begin{array}{ll}278 & 1328.54 \\ 278 & 1333.54\end{array}$

$278 \quad 1338.54$

$278 \quad 1343.54$

$278 \quad 1353.54$

\begin{tabular}{ll}
278 & 1358.54 \\
278 & 1403.54 \\
\hline
\end{tabular}
48.37320
48.35820

48.34300

48.32740

48.31180
48.29620

48.29620
48.28090

48.26560
48.25030

48.23370

48.21070
48.19010

48.17036
40.10250

48.14850

48.13320
48.11790

48.10260
48.08736

48.07210

48.04684

48.02440
48.00880

47.99350
47.97830

47.90300

47.94770
47.93240

47.91710
47.90180

47.88050

47.85590
47.84050

47.82530
47.81000

47.81000
47.79400

47.77950
47.76470

47.76470
47.75100
47.73730

47.73730
47.72300

47.70990
47.69020

47.68250
47.60730

47.062500
47.63680

47.63680
47.62150

47.60620
47.59090

47.59090
47.57550
47.50010

173.03120
173.02650

173.01770

173.01030
173.00290

172.99560
172.98860

172.98860
172.98160

172.97460
172.96770
172.96070

172.94940

172.94310

172.93080
172.93050
172.92350

172.92350
172.41050

172.90960
172.90280

172.89590

172.88910
172.88260

172.87010
172.87010
172.87620

172.86420
172.85830

172.85830
172.85250

172.84660
172.84080
172.83690

172.82910

172.82320
17281730

172.81160
172.80000

172.80030
172.79400

172.78880
172.78310

172.78310
172.77710
172.77000

172.77060
172.76400
172.75740

172.75740
172.75080
172.74430

172.74430
172

17284.0
172.7370058908

$\begin{array}{ll}172.73270 & 5544.0 \\ 172.72780 & 5435.3 \\ 172.72290 & 5408.3\end{array}$

172.72290
172.71790

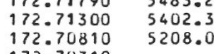

5168.2

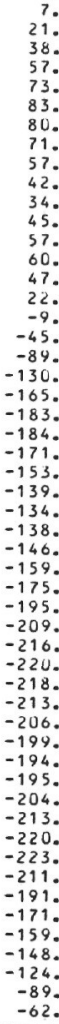

\begin{tabular}{|c|c|}
\hline $\begin{array}{r}23.9 \\
-3.9\end{array}$ & 374.2 \\
\hline $\begin{array}{l}-18.8 \\
-15.3\end{array}$ & $\begin{array}{l}385.7 \\
392.0\end{array}$ \\
\hline $\begin{array}{l}-14.0 \\
-12.1\end{array}$ & $\begin{array}{l}391.3 \\
394 .\end{array}$ \\
\hline & \\
\hline-8.7 & 390.9 \\
\hline & $\begin{array}{l}393.1 \\
393.7\end{array}$ \\
\hline $\begin{array}{l}-0.1 \\
-5.5\end{array}$ & $\begin{array}{l}395.7 \\
393.7\end{array}$ \\
\hline 3. & 392.5 \\
\hline & 385.5 \\
\hline $\begin{array}{r}-1.4 \\
3.6\end{array}$ & $\begin{array}{l}385.0 \\
387.2\end{array}$ \\
\hline 0.0 & 39001 \\
\hline & $\begin{array}{l}3911.8 \\
393.7\end{array}$ \\
\hline & \\
\hline 11.4 & 391.3 \\
\hline $\begin{array}{l}13.2 \\
13.6\end{array}$ & $\begin{array}{r}388.0 \\
3946\end{array}$ \\
\hline $\begin{array}{l}13 . \\
15 .\end{array}$ & $\begin{array}{r}59460 \\
400.0\end{array}$ \\
\hline 16. & $\begin{array}{l}402.5 \\
6027\end{array}$ \\
\hline 16. & $\begin{array}{l}402.7 \\
399.6\end{array}$ \\
\hline 16. & 398.5 \\
\hline $150^{\circ}$ & $\begin{array}{l}396.7 \\
389.4\end{array}$ \\
\hline & \\
\hline .0 & 403.8 \\
\hline $\begin{array}{l}16.3 \\
16.5\end{array}$ & \\
\hline 16. & 401.6 \\
\hline & $\begin{array}{l}40253 \\
395.7\end{array}$ \\
\hline & 395.7 \\
\hline & $\begin{array}{l}401.5 \\
3975\end{array}$ \\
\hline & $\begin{array}{r}397.5 \\
385.9\end{array}$ \\
\hline & \\
\hline
\end{tabular}


TABLE 3 - Continued

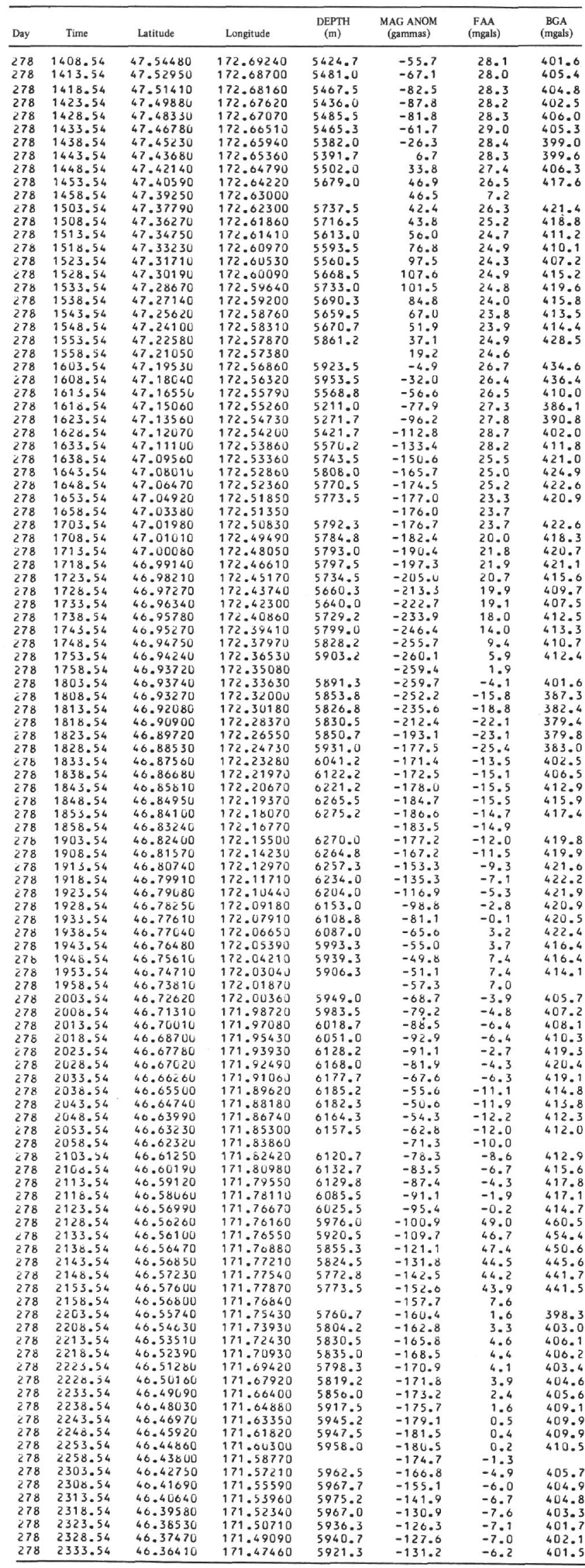

TABLE 3 - Continued

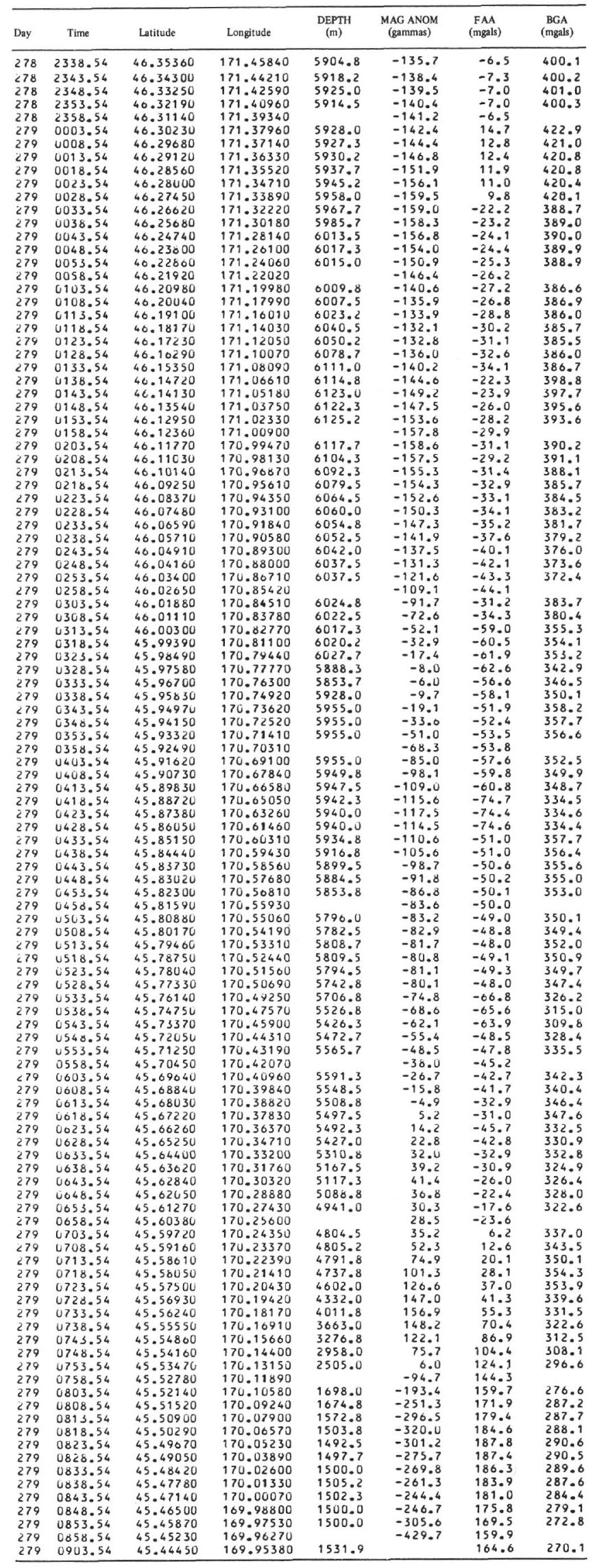


PRE-LEG 55 SITE SURVEY GEOPHYSICAL DATA

TABLE 3 - Continued

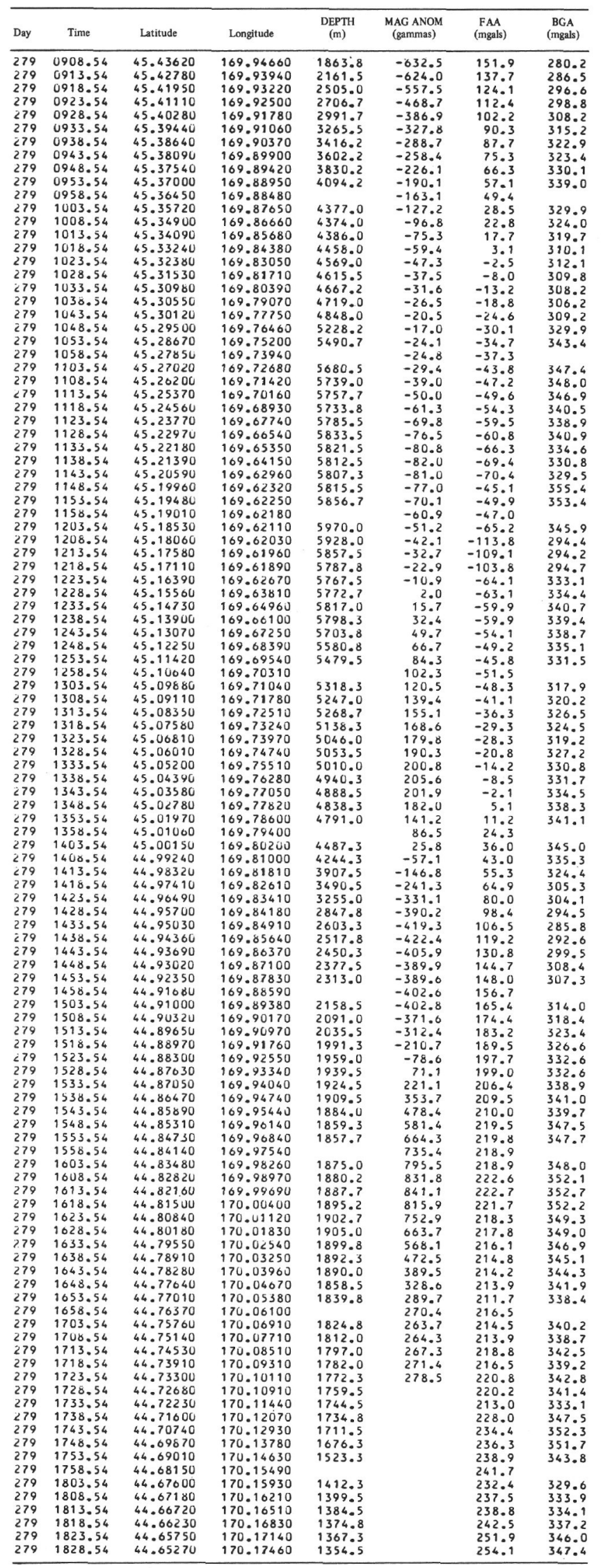

TABLE 3 - Continued

\begin{tabular}{|c|c|c|c|c|c|c|c|}
\hline Day & Time & Latitude & Longitude & $\underset{(\mathrm{m})}{\mathrm{DEPTH}}$ & $\underset{\text { (gac ANmas) }}{\text { MACM }}$ & $\begin{array}{c}\text { FAA } \\
\text { (mgals) }\end{array}$ & $\begin{array}{c}\text { BGA } \\
\text { (mgals) }\end{array}$ \\
\hline & & 44.64830 & & 1350.0 & & 256.3 & \\
\hline 279 & 1838.54 & 44.64410 & 170,18070 & 1334.3 & & 260.6 & 352. \\
\hline 279 & 1843.54 & 44.63870 & 170.18490 & 1317.0 & & 269.2 & 359. \\
\hline 279 & 1848.54 & 44.63270 & 170.18950 & 1312.5 & & 278.3 & 368.7 \\
\hline $\begin{array}{l}279 \\
279\end{array}$ & $\begin{array}{l}1853.54 \\
1858.54\end{array}$ & $\begin{array}{l}44.62670 \\
44.62070\end{array}$ & $\begin{array}{l}170.19420 \\
170.19890\end{array}$ & & & $\begin{array}{l}282.9 \\
282.7\end{array}$ & \\
\hline 279 & 1903.54 & 44.61550 & 170.20330 & 1257.0 & & $\begin{array}{l}282.5 \\
286.5\end{array}$ & \\
\hline & 1908.54 & 44.61060 & 170.20740 & & & 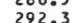 & \\
\hline 279 & 1913.54 & 44.60580 & 170.21160 & 1239.8 & & 291.9 & 377. \\
\hline 279 & 1918.54 & 44.60140 & 170.21580 & 1237.5 & & 299.3 & 384. \\
\hline 279 & 1923.54 & 44.59740 & 170.22010 & 1227.0 & & 301.8 & 386. \\
\hline $\begin{array}{l}279 \\
279\end{array}$ & $\begin{array}{r}1928.54 \\
1933.54\end{array}$ & $\begin{array}{r}44.59340 \\
44.58950\end{array}$ & & 1206.8 & & 301.8 & 384.5 \\
\hline 279 & $\begin{array}{r}1933.54 \\
1938.54\end{array}$ & $\begin{array}{l}44.58950 \\
44.58550\end{array}$ & $\begin{array}{r}170.22880 \\
170.23300\end{array}$ & $\begin{array}{l}1194.8 \\
1176.8\end{array}$ & & $\begin{array}{l}307.5 \\
309.8\end{array}$ & 389.8 \\
\hline 279 & 1943.54 & 44.58100 & 170.23720 & 1159.5 & & 313.1 & 392. \\
\hline & 1948.54 & 44.57700 & 170.24150 & 1134.0 & & 316.5 & 394.6 \\
\hline 279 & 1953.54 & 44.57360 & 170.24580 & 1114.5 & & 318.0 & 394.7 \\
\hline 279 & 1958.54 & & 170.25010 & & & 318.5 & \\
\hline $\begin{array}{l}279 \\
279\end{array}$ & $\begin{array}{r}2003.54 \\
2008.54\end{array}$ & $\begin{array}{r}66.56570 \\
44.56170\end{array}$ & $\begin{array}{l}170.25440 \\
170.25870\end{array}$ & $1084 \cdot 5$ & & 316.2 & 390.5 \\
\hline & $\begin{array}{l}2008.54 \\
2013.54\end{array}$ & $\begin{array}{l}44.56170 \\
44.55780\end{array}$ & 170.26300 & $\begin{array}{l}1085.2 \\
1098.0\end{array}$ & & & $\begin{array}{l}394.5 \\
394.9\end{array}$ \\
\hline 279 & 2018.54 & 44.5530 & 170.26730 & & & 320.0 & $390^{\circ}$ \\
\hline 279 & 2023.54 & 44.54990 & 170.27150 & 1089.8 & & 321.1 & 396.1 \\
\hline 279 & 2030.06 & 44.54690 & 170.27700 & 1072 & & & \\
\hline
\end{tabular}

279
279
2030.035 .06

$\begin{array}{ll}279 & 2040.04 \\ 279 & 2045.04\end{array}$

$\begin{array}{ll}279 & 2050.04 \\ 279 & 2055.04\end{array}$

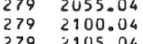

$279 \quad 2110.04$

$\begin{array}{ll}2799 & 21115.04 \\ 2720.04 & 2204\end{array}$

$\begin{array}{ll}279 \\ 279 & 2125.04 \\ 2730.04\end{array}$

$\begin{array}{ll}279 & 2135.04 \\ 279 & 2140.04 \\ 279 & 2155.04\end{array}$

$279 \begin{aligned} & 2145.04 \\ & 2150.04\end{aligned}$

$\begin{array}{ll}279 & 2155.04 \\ 279 & 2200.04\end{array}$

$279 \quad 2205.04$

$\begin{array}{rr}279 & 2210.04 \\ 279 & 2215.04 \\ 2790 & 2225.04\end{array}$

$\begin{array}{ll}279 & 2220.04 \\ 279 & 2225.04 \\ 279 & 2235\end{array}$

$\begin{array}{rl}279 & 2230.04 \\ 279 & 2235.04\end{array}$

$\begin{array}{ll}279 & 2240.04 \\ 279 & 2245.04\end{array}$

27922250.04

$\begin{array}{ll}279 & 2255.04 \\ 279 & 2300.04\end{array}$

$\begin{array}{ll}279 & 2305.04 \\ 279 & 2310.04\end{array}$

$\begin{array}{ll}279 & 2315.04 \\ 270 & 2320.04 \\ 279 & 235.04\end{array}$

$\begin{array}{ll}279 & 2325.04 \\ 279 & 2330.06\end{array}$

$\begin{array}{ll}279 & 2355.04 \\ 279 & 2340.04 \\ 279 & 235.04\end{array}$

$\begin{array}{ll}279 & 2340.04 \\ 279 & 2345.04 \\ 279 & 335.04\end{array}$

$\begin{array}{ll}279 & 2350.04 \\ 2355.04\end{array}$

2800005.04

$280 \quad 6015.04$

$\begin{array}{ll}280 & 020.04 \\ 280 & 0025.04\end{array}$

$\begin{array}{ll}280 & 0025.04 \\ 280 & 0030.04\end{array}$

$\begin{array}{ll}280 & 0035.04 \\ 280 & 0040.04 \\ 280 & 0050.04\end{array}$

2800045.04

$280 \quad 0055.04$

$\begin{array}{ll}280 & 0100.04 \\ 280 & 0105.04\end{array}$

$\begin{array}{ll}280 & 0110.04 \\ 280 & 0115.04\end{array}$

$\begin{array}{ll}280 & 0120.04 \\ 280 & 0125.04\end{array}$

$\begin{array}{ll}280 & 0125.04 \\ 280 & 0130.04 \\ 280 & 0135\end{array}$

$280 \quad 0143.04$

$\begin{array}{ll}280 & 0150.04 \\ 280 & 0155.04\end{array}$

$80 \quad 0200.04$

$\begin{array}{ll}280 & 0205.04 \\ 280 & 0210.04 \\ 280 & 025.04\end{array}$

$\begin{array}{ll}280 & 0225.04 \\ 280 & 0220.06\end{array}$

2800225.04

$\begin{array}{ll}280 & 02350.04 \\ 280 & 0235.04 \\ 280 & 0245.04\end{array}$

$\begin{array}{ll}280 & 0240.04 \\ 280 & 0245.04\end{array}$

$\begin{array}{ll}2880 & 0245.04 \\ 280 & 0250.04\end{array}$

$280 \quad 0300.04$

$280 \quad 0305.04$

$\begin{array}{ll}280 & 3330.04 \\ 280 & 0315.04\end{array}$

$\begin{array}{ll}280 & 0320.04 \\ 280 & 0325.04\end{array}$

$280 \quad 0330.04$

28000340.04

$280 \quad 0345.04$

\begin{tabular}{ll}
280 & 0355.04 \\
280 & 0600.04 \\
\hline
\end{tabular}

44.54690

44.52610
44.51670

44.50740

0.170 .288350
1095.0

$44.49090 \quad 170.29070 \quad 1110.0$

$\begin{array}{lll}44.49220 & 170.29070 & 1125.0 \\ 44.49340 & 170.30270 & 1110.0\end{array}$

$\begin{array}{lll}44.04460 & 1700.30860 & 1140.0 \\ 44.49580 & 170.31460 & 1147.5\end{array}$

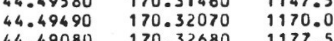

$\begin{array}{lll}44.48080 & 170.32680 & 1177.5 \\ 44.33300 & 1192.5\end{array}$

$66.67860 \quad 170.33910 \quad 1207.5$

$\begin{array}{lll}44.47450 & 170.35140 & 1222.5\end{array}$

$\begin{array}{lll}44.46540 & 170.35650 & 1260.0 \\ 45980 & 1275.0\end{array}$

$44.46060 \quad 170.36320 \quad 1290.0$

$\begin{array}{lll}44.455110 & 170.360600 & 1297.5 \\ 44.44040 & 170.373400 & 1320.0 \\ 4327.5\end{array}$

$\begin{array}{lll}44.44440 & 170.37340 & 1327.5 \\ 44.3760 & 170.37670 & 1342.5 \\ 4.43370 & 170.37820 & 1372.5\end{array}$

$\begin{array}{lll}44.42580 & 170.37970 & 1372.5 \\ 44.4790 & 170038110 & 1522.5\end{array}$

$\begin{array}{lll}44.41790 & 170.38110 & 1522.5 \\ 44.40990 & 170.38260 & 1575.0\end{array}$

$44.40200 \quad 170.38410 \quad 1612.5$

$\begin{array}{lll}44.39410 & 170.30550 & 1627.5 \\ 44.38290 & 170.38350 & 1650.0\end{array}$

$44.37160 \quad 170.38150 \quad 1672.5$

$\begin{array}{lll}44.36640 & 1700.37940 & 1717.5 \\ 44.34910 & 170.37740 & 1762.5\end{array}$

$\begin{array}{lll}44.33790 & 170.37540 & 1792.5 \\ 44.32660 & 170.37330 & 1807.5\end{array}$

$44.31080 \quad 170.37350 \quad 1837.5$

$\begin{array}{lll}44.30700 & 170.37360 & 1860.0 \\ 44.29710 & 170.37370 & 1875.0\end{array}$

$\begin{array}{lll}44.28730 & 170.37390 & 1882.5\end{array}$

$\begin{array}{lll}44.2750 & 170.37400 & 1890.0 \\ 44.26770 & 170.37420 & 1912.5\end{array}$

$\begin{array}{lll}44.25790 & 170.37430 & 1927.5 \\ 44.24810 & 170.37440 & 1927.5\end{array}$

$44.23620 \quad 170.37460 \quad 1935.0$

$\begin{array}{lll}44.22840 & 170.37470 & 1920.0 \\ 44.21860 & 170.37480 & 1897.5\end{array}$

$\begin{array}{lll}44.20880 & 170.37480 & 1897.5 \\ 44.37500 & 1867.5\end{array}$

$\begin{array}{lll}44.19870 & 170.37500 & 1815.0 \\ 44.18800 & 170.37510 & 1792.5\end{array}$

$44.17850 \quad 170.37510 \quad 1762.5$

$\begin{array}{lll}44.15840 & 170.37520 & 1760.0 \\ 44520 & 1785.0\end{array}$

$\begin{array}{lll}44.14830 & 170.37520 & 1852.5 \\ 44.13940 & 170.37260 & 1935.0\end{array}$

44.13350
44.12150

(460.36720

$44.10370 \quad 170.30180 \quad 2130.0$

$\begin{array}{ll}44.09480 & 170.35910 \quad 2497.5\end{array}$

$\begin{array}{lll}44.06110 & 170.36280 & 2827.5 \\ 170.36870 & 3225.0\end{array}$

$44.04300 \quad 170.37380 \quad 3292.5$

$\begin{array}{lll}44.03010 & 170.37740 & 3390.0 \\ 44.01710 & 170.36110 & 3442.5\end{array}$

$\begin{array}{lll}44.00530 & 170.378050 & 3742.5\end{array}$

$\begin{array}{lll}43.99350 & 170.37990 & 4012.5 \\ 43.98170 & 170.37930 & 4417.5\end{array}$

$\begin{array}{lll}43.96990 & 170.37870 & 4717.5 \\ 43.95800 & 170.37810 & 5317.5\end{array}$

$\begin{array}{lll}43.94020 & 170.37810 & 5317.5 \\ 43.93440 & 170.37590 & 5392.5 \\ 4385.0 & 5890\end{array}$

$\begin{array}{lll}43.92200 & 170.37690 & 5385.0 \\ 43150 & 5415.0\end{array}$

$\begin{array}{lll}43.91140 & 170.37570 & 5362.5 \\ 43.90050 & 170.37510 & 5257.5 \\ 43.8700 & 170.37400 & 5197.5\end{array}$

$\begin{array}{lll}43.88960 & 170.37440 & 5197.5 \\ 43.87880 & 170.37380 & 5167.5\end{array}$

$\begin{array}{lll}43.87880 & 170.37380 & 5167.5 \\ 43.86640 & 170.37510 & 5032.5\end{array}$

$\begin{array}{lll}43.86640 & 170.37510 & 5032.5 \\ 43.85410 & 170.37630 & 4875.0 \\ 43.8480 & 170.37750 & 4717.5\end{array}$

$43.82930 \quad 170.37890 \quad 4657.5$

$43.81690 \quad 170.38020$
43840600

$\begin{array}{lll}43.80440 & 170.38150 & 4395.0 \\ 43.79150 & 170.38020 & 4530.0\end{array}$

$\begin{array}{lll}43.77860 & 1700.37890 & 4545.0 \\ 43.76520 & 170.37750 & 4470.0\end{array}$

$\begin{array}{lll}43.76520 & 170.37750 & 4470.0 \\ 43.75000 & 170.37610 & 4320.0\end{array}$

$\begin{array}{lll}43.73680 & 170.37470 & 4185.0 \\ 43.71950 & 170.37320 & 3975.0\end{array}$ 
TABLE 3 - Continued

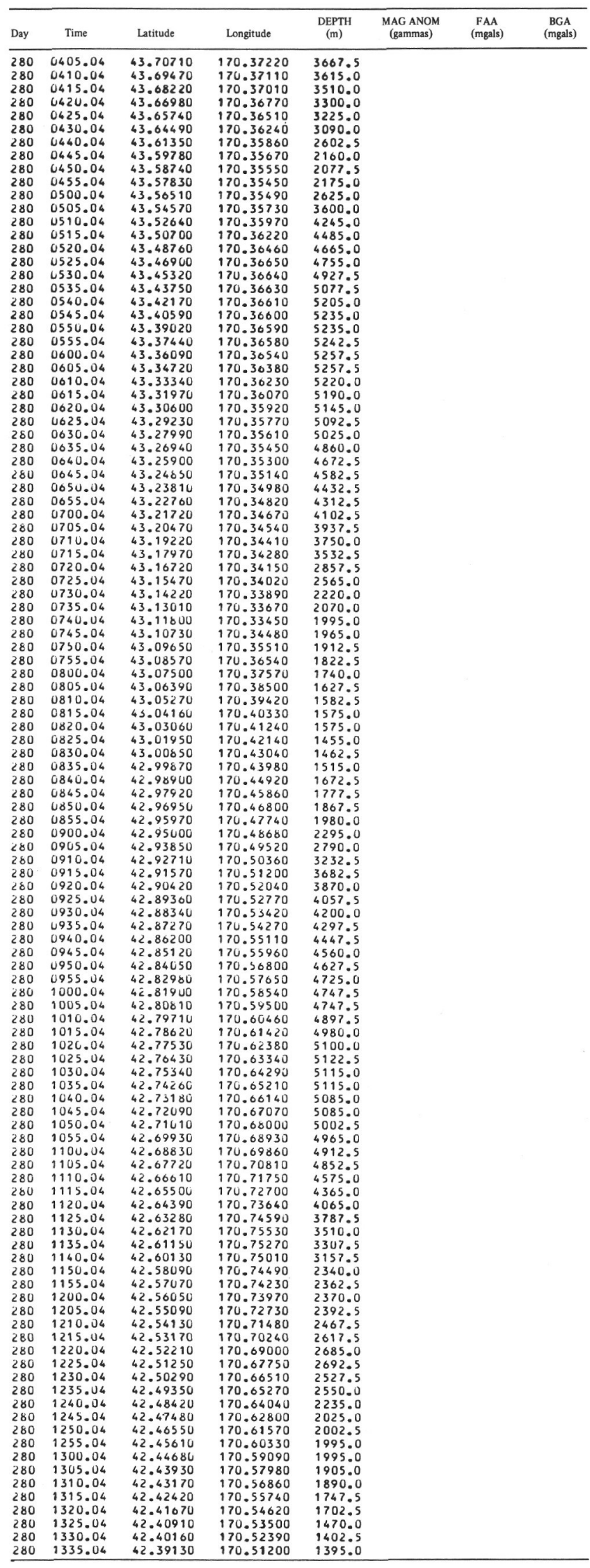

TABLE 3 - Continued

\begin{tabular}{|c|c|c|c|c|c|c|c|}
\hline Day & Time & Latitude & Longitude & $\underset{\substack{\mathrm{Dm}) \\
\text { DEPTH }}}{ }$ & $\underset{\text { (gammas) }}{\operatorname{MAG~ANOM}}$ & $\begin{array}{c}\text { FAA } \\
\text { (mgals) }\end{array}$ & $\begin{array}{c}\text { BGA } \\
\text { (mgals) }\end{array}$ \\
\hline 280 & 1340.04 & 42.38080 & 170.50030 & 1380.0 & & & \\
\hline & $\begin{array}{l}1350.04 \\
350.04\end{array}$ & $\begin{array}{l}42.35020 \\
42.35970\end{array}$ & $\begin{array}{l}170.048800 \\
770.47700\end{array}$ & $\begin{array}{r}1335.0 \\
1335.0\end{array}$ & & & \\
\hline $\begin{array}{l}280 \\
280\end{array}$ & $\begin{array}{l}1355.04 \\
1400.04\end{array}$ & $\begin{array}{r}42.34920 \\
62.33800\end{array}$ & $\begin{array}{l}170.605300 \\
770.45360\end{array}$ & $\begin{array}{l}1312.5 \\
1290.0\end{array}$ & & & \\
\hline $\begin{array}{l}280 \\
280 \\
280\end{array}$ & $\begin{array}{l}1405.04 \\
140.04\end{array}$ & $\begin{array}{r}62.32810 \\
42.31770\end{array}$ & $\begin{array}{l}170.4200 \\
70.4300\end{array}$ & $\begin{array}{l}1265.0 \\
1192.5\end{array}$ & & & \\
\hline $\begin{array}{l}280 \\
280 \\
280\end{array}$ & $\begin{array}{l}1415.04 \\
1420.04\end{array}$ & $\begin{array}{r}42.30730 \\
42.29690\end{array}$ & $\begin{array}{l}170.41920 \\
170.40790\end{array}$ & $\begin{array}{r}11110.0 \\
982.5\end{array}$ & & & \\
\hline $\begin{array}{l}280 \\
280 \\
280\end{array}$ & $\begin{array}{l}145.04 \\
1430.04\end{array}$ & $\begin{array}{r}\$ 2.28560 \\
42.276010\end{array}$ & $\begin{array}{l}170.393660 \\
770.38520\end{array}$ & $\begin{array}{l}1132.5 \\
1245: 0\end{array}$ & & & \\
\hline & $\begin{array}{l}1430.04 \\
1440.04\end{array}$ & $\begin{array}{l}42.26050 \\
42.26210\end{array}$ & $\begin{array}{r}170.37190 \\
7703700\end{array}$ & $\begin{array}{l}1355.0 \\
1087.5\end{array}$ & & & \\
\hline 280 & $\begin{array}{l}145.06 .06 \\
1450.04\end{array}$ & $\begin{array}{r}2.253900 \\
42.25600\end{array}$ & $\begin{array}{r}70.37010 \\
770.36800\end{array}$ & $\begin{array}{r}1327.5 \\
1342.5\end{array}$ & & & \\
\hline & $\begin{array}{l}1455.04 \\
1500.04\end{array}$ & $\begin{array}{l}42.2525200 \\
42.24990\end{array}$ & $\begin{array}{l}170.060 \\
170\end{array}$ & $\begin{array}{l}1380.0 \\
1387.5\end{array}$ & & & \\
\hline & $\begin{array}{l}15505.04 \\
1510.04\end{array}$ & $\begin{array}{l}42.23770 \\
42.22560\end{array}$ & $\begin{array}{l}170.035030 \\
770.33500\end{array}$ & $\begin{array}{l}10202.5 \\
1432.5\end{array}$ & & & \\
\hline $\begin{array}{l}280 \\
280 \\
280\end{array}$ & $\begin{array}{l}1555.04 \\
1520.06\end{array}$ & $\begin{array}{l}42.21340 \\
42.20130\end{array}$ & $\begin{array}{l}170.31970 \\
170,3040\end{array}$ & $\begin{array}{l}1492.5 \\
1717.5\end{array}$ & & & \\
\hline $\begin{array}{l}280 \\
280 \\
280\end{array}$ & $\begin{array}{l}1525.04 \\
1530.04\end{array}$ & $\begin{array}{r}42.18910 \\
42.17700\end{array}$ & $\begin{array}{l}170.28910 \\
170.27375\end{array}$ & $\begin{array}{l}1957.5 \\
2767.5\end{array}$ & & & \\
\hline $\begin{array}{l}280 \\
280\end{array}$ & $\begin{array}{l}1535.04 \\
1540.04\end{array}$ & $\begin{array}{l}42.16060 \\
42.15530\end{array}$ & $\begin{array}{l}170.25800 \\
17022420\end{array}$ & $\begin{array}{l}3337.5 \\
3472.5\end{array}$ & & & \\
\hline $\begin{array}{l}280 \\
280 \\
280\end{array}$ & $\begin{array}{l}1554.04 .04 \\
1550.04\end{array}$ & $\begin{array}{l}42.14380 \\
42.13180\end{array}$ & $\begin{array}{l}170.22560 \\
770.20840\end{array}$ & $\begin{array}{l}3592.5 \\
3705.0\end{array}$ & & & \\
\hline & $\begin{array}{l}1555.04 \\
1600.04\end{array}$ & $\begin{array}{l}42.11980 \\
42.10770\end{array}$ & $\begin{array}{l}170: 09110 \\
770: 17390\end{array}$ & $\begin{array}{l}3757.5 \\
3952.5\end{array}$ & & & \\
\hline $\begin{array}{l}280 \\
<80 \\
<80\end{array}$ & $\begin{array}{l}1008.54 \\
1613.54\end{array}$ & $\begin{array}{r}22.08990 \\
42.02940\end{array}$ & $\begin{array}{l}170.0150 \\
170\end{array}$ & $\begin{array}{l}215.5 \\
4657.2\end{array}$ & & $\begin{array}{r}4.1 \\
-13.8\end{array}$ & $\begin{array}{l}2964.4 \\
293.1\end{array}$ \\
\hline $\begin{array}{l}280 \\
280 \\
280\end{array}$ & $\begin{array}{l}1618.54 \\
1623.54\end{array}$ & $\begin{array}{l}42.008890 \\
42.05830\end{array}$ & $\begin{array}{l}170.12540 \\
170.111240\end{array}$ & $\begin{array}{r}4500.7 \\
4625.2\end{array}$ & & & $\begin{array}{l}98.7 \\
48.1\end{array}$ \\
\hline & $\begin{array}{l}1628.54 \\
1633.54\end{array}$ & $\begin{array}{l}42.04780 \\
42.03830\end{array}$ & $\begin{array}{l}170.09930 \\
170 \\
708300\end{array}$ & $\begin{array}{r}4938.7 \\
5288.2\end{array}$ & & $\begin{array}{l}-33.9 \\
-49.8\end{array}$ & 306.2 \\
\hline & 1638.54 & & 170.06730 & 5379.8 & & -56.9 & \\
\hline $\begin{array}{l}280 \\
280\end{array}$ & $\begin{array}{l}1643.54 \\
1648.54\end{array}$ & $\begin{array}{r}42.022020 \\
42.00980\end{array}$ & $\begin{array}{l}170.05080 \\
170.03690\end{array}$ & $\begin{array}{l}5372.3 \\
5364: 8\end{array}$ & & $\begin{array}{l}-60.4 \\
-82.5\end{array}$ & $\begin{array}{l}309.5 \\
280.9\end{array}$ \\
\hline & $\begin{array}{l}1053.54 \\
1658.54\end{array}$ & $\begin{array}{l}41.99910 \\
41.98850\end{array}$ & $\begin{array}{l}170.023200 \\
170.00950\end{array}$ & 5357.3 & & $\begin{array}{l}-655.4 \\
-62.8\end{array}$ & \\
\hline $\begin{array}{l}286 \\
280 \\
280\end{array}$ & $\begin{array}{l}1703.54 \\
1708.54\end{array}$ & $\begin{array}{l}41.97800 \\
41.96950\end{array}$ & 169.99800 & $\begin{array}{r}5365.5 \\
5506 .\end{array}$ & & & 17.3 \\
\hline & 1713.54 & 41.96020 & 169.97680 & 5485.5 & & -55.0 & 22.7 \\
\hline & $\begin{array}{l}7718.54 \\
1723.54\end{array}$ & $\begin{array}{l}47.95000 \\
41.94050\end{array}$ & $\begin{array}{l}16990965800 \\
1099530\end{array}$ & $\begin{array}{l}5554.5 \\
5583: 0\end{array}$ & & $\begin{array}{l}-60.8 \\
-61.0\end{array}$ & \\
\hline $\begin{array}{l}280 \\
280 \\
280\end{array}$ & $\begin{array}{l}1728.54 \\
1733.54\end{array}$ & $\begin{array}{l}41.9500 \\
41.92080\end{array}$ & 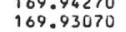 & $\begin{array}{l}5692.7 \\
5600.2\end{array}$ & & $\begin{array}{l}-60.0 \\
-62.8\end{array}$ & \\
\hline & 1738.54 & 41.91110 & 169.91850 & $\begin{array}{r}5602.5 \\
5592.5\end{array}$ & & -61.9 & $\begin{array}{l}323.9 \\
3246\end{array}$ \\
\hline $\begin{array}{l}280 \\
280 \\
280\end{array}$ & $\begin{array}{l}1788.56 \\
1753.54\end{array}$ & $\begin{array}{l}41.89030 \\
41.8770\end{array}$ & $\begin{array}{l}1099.90250 \\
16990700\end{array}$ & $\begin{array}{l}5526.8 \\
5508.0\end{array}$ & & $\begin{array}{l}-15.6 \\
-79.2\end{array}$ & $\begin{array}{l}365.0 \\
300.1\end{array}$ \\
\hline $\begin{array}{l}280 \\
280 \\
280\end{array}$ & $\begin{array}{l}1758.54 \\
1803.54\end{array}$ & $\begin{array}{l}41.80520 \\
41.85550\end{array}$ & $\begin{array}{l}109.991150 \\
10999930\end{array}$ & 5311.5 & & $\begin{array}{l}-79.7 \\
-58.4\end{array}$ & \\
\hline & 1808.54 & 41.84720 & 109.92840 & 5299.5 & & -56.7 & 308.2 \\
\hline & $\begin{array}{l}1813.54 \\
1818.54\end{array}$ & 41.83050 & $\begin{array}{l}169.938760 \\
169\end{array}$ & $\begin{array}{l}54040.0 \\
5404.5\end{array}$ & & $\begin{array}{l}-32.8 \\
-49.0\end{array}$ & $\begin{array}{l}315.5 \\
323.2\end{array}$ \\
\hline & & $\begin{array}{l}41.82000 \\
41.80840\end{array}$ & $\begin{array}{l}109.995560 \\
16990790\end{array}$ & $\begin{array}{l}54212.0 \\
5339.3\end{array}$ & & $\begin{array}{r}-44.6 \\
-42.9\end{array}$ & $\begin{array}{l}323.1 \\
334.8\end{array}$ \\
\hline 280 & 1833.54 & 41.79620 & 169.97130 & 5094.8 & & -39.3 & 311.5 \\
\hline 280 & $\begin{array}{r}1888684 \\
1863.54\end{array}$ & 41.77190 & $\begin{array}{l}169.9898610 \\
1690\end{array}$ & $\begin{array}{l}8600.8 \\
4679.3\end{array}$ & & $\begin{array}{l}-35.6 \\
-28.6\end{array}$ & $\begin{array}{l}299.1 \\
293.6\end{array}$ \\
\hline 280 & $\begin{array}{l}1853.54 \\
853.54\end{array}$ & $\begin{array}{l}41.7450 \\
41.7250\end{array}$ & $\begin{array}{l}179.0000090 \\
70.000\end{array}$ & 4470.0 & & $\begin{array}{l}-25.1 \\
-17.9\end{array}$ & 289.9 \\
\hline 280 & 1903054 & 41.72520 & 170.01590 & 4638.7 & & $\begin{array}{l}-11.6 \\
-11.4\end{array}$ & 308.0 \\
\hline $\begin{array}{l}280 \\
280\end{array}$ & 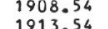 & 41.71640 & $\begin{array}{l}70.02350 \\
170\end{array}$ & 4654.5 & & -6 & \\
\hline & & 41.69280 & $170: 03860$ & 4301.3 & & & \\
\hline $\begin{array}{l}280 \\
280 \\
280\end{array}$ & $\begin{array}{r}1923.54 \\
1928.54\end{array}$ & $\begin{array}{l}41.08200 \\
41.67140\end{array}$ & $\begin{array}{l}170.04620 \\
170.05420\end{array}$ & $\begin{array}{l}4058.3 \\
3960.88\end{array}$ & & 16.9 & $\begin{array}{l}280.4 \\
2888\end{array}$ \\
\hline $\begin{array}{l}280 \\
280\end{array}$ & $\begin{array}{l}1933.55 \\
1938.54\end{array}$ & $\begin{array}{l}41.66120 \\
41.65090\end{array}$ & 170.00360 & $\begin{array}{l}3682.5 \\
37590.0\end{array}$ & & $\begin{array}{r}20.7 \\
21.5\end{array}$ & 2803 \\
\hline & .54 & 41.64000 & 170.08120 & 3806.2 & & & 280.3 \\
\hline $\begin{array}{l}280 \\
280\end{array}$ & $\begin{array}{l}1948.54 \\
1953.54\end{array}$ & $\begin{array}{l}41.63000 \\
41.62010\end{array}$ & $\begin{array}{l}170.0902020 \\
170.099910\end{array}$ & $\begin{array}{l}3982.5 \\
4032.7\end{array}$ & & $\begin{array}{l}22.2 \\
22.8\end{array}$ & $\begin{array}{l}296.4 \\
300.5\end{array}$ \\
\hline & $\begin{array}{l}1958.54 \\
20035.54\end{array}$ & $\begin{array}{r}41.6090 \\
41.60030\end{array}$ & $\begin{array}{l}70.08000 \\
170\end{array}$ & 4025. & & & \\
\hline 280 & 2008.54 & 41.59110 & 580 & 4116.7 & & & 299.5 \\
\hline 280 & $\begin{array}{l}2018.58 \\
2018.54\end{array}$ & $\begin{array}{l}41.58180 \\
41.57250\end{array}$ & $\begin{array}{l}770.13460 \\
170.14350\end{array}$ & $\begin{array}{r}40860.8 \\
3973.5\end{array}$ & & $\begin{array}{l}\begin{array}{l}21.8 \\
23.4\end{array} \\
23.4\end{array}$ & $\begin{array}{l}303.2 \\
297.0\end{array}$ \\
\hline 280 & $\begin{array}{l}2023.54 \\
2028.54\end{array}$ & $\begin{array}{l}41.56330 \\
41.59400\end{array}$ & $\begin{array}{l}70.015260 \\
170.16120\end{array}$ & $\begin{array}{l}3795.8 \\
3650.3\end{array}$ & & & \\
\hline & 2033.54 & 86 & & 3501.8 & & & \\
\hline $\begin{array}{l}280 \\
280\end{array}$ & $\begin{array}{l}2038.54 \\
2043.54\end{array}$ & $\begin{array}{l}41.5330 \\
41.52260\end{array}$ & $\begin{array}{l}770.18090 \\
170 \\
19990\end{array}$ & $\begin{array}{l}3345.0 \\
3352.5\end{array}$ & & $\begin{array}{l}43.9 \\
48.6\end{array}$ & $\begin{array}{l}277.2 \\
279.5\end{array}$ \\
\hline & $\begin{array}{l}20680.54 \\
2053.54\end{array}$ & $\begin{array}{l}41.51170 \\
41.50100\end{array}$ & $\begin{array}{l}170.20090 \\
170.21090\end{array}$ & $\begin{array}{l}3364.5 \\
3276.0\end{array}$ & & $57 \quad$ & $\begin{array}{l}285.1 \\
282.8\end{array}$ \\
\hline 800 & 2058.5 & 41.49030 & 170.22090 & & & 63 & \\
\hline $\begin{array}{l}280 \\
280\end{array}$ & $\begin{array}{l}2103.54 \\
2108.54\end{array}$ & $\begin{array}{l}41.48100 \\
41.47240\end{array}$ & $\begin{array}{l}170.23150 \\
170.24220\end{array}$ & $\begin{array}{l}2928.0 \\
2648.3\end{array}$ & & $\begin{array}{l}70.6 \\
77.6\end{array}$ & \\
\hline & 113. & $\begin{array}{l}41.46830 \\
41.45820\end{array}$ & $\begin{array}{l}770.252590 \\
170.26300\end{array}$ & $\begin{array}{l}2538.8 \\
2417.3\end{array}$ & & & 2577 \\
\hline & 2123.54 & 60 & 170.27440 & 2317.5 & & 101.4 & \\
\hline 280 & $\begin{array}{l}2138.54 \\
213.54\end{array}$ & 41.42870 & 170.29420 & $\begin{array}{l}2158.5 \\
2000.3\end{array}$ & & $\begin{array}{l}109.6 \\
113.6\end{array}$ & 251.3 \\
\hline & 1.63 .54 & (6070 & $\begin{array}{l}170.34 \\
170.31\end{array}$ & $\begin{array}{l}191 \\
190\end{array}$ & & & \\
\hline & 8.54 & & 170.31950 & 1823.3 & & & \\
\hline & 年 & 8810 & 170.3360 & 10320 & & & \\
\hline & 2203.54 & 37070 & 170.3 & 13 & & & \\
\hline 280 & 2213.54 & $\begin{aligned} 41.34900 \\
\end{aligned}$ & 170.36010 & 13340.3 & & 185.5 & \\
\hline 280 & & 41.32630 & 170.37590 & 130 & & & \\
\hline 280 & 8.54 & 41.31460 & 170.38390 & 1287.0 & & & \\
\hline 280 & 2238.54 & 41.29070 & $\begin{array}{r}170.39980 \\
170\end{array}$ & $\begin{array}{l}1260.8 \\
124.3\end{array}$ & & 218.5 & $\begin{array}{l}94.2 \\
04.8\end{array}$ \\
\hline 年 & & 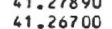 & $\begin{array}{l}170.4 \\
170.4\end{array}$ & 119 & & & \\
\hline & 3.54 & 15500 & 170.42360 & 1164.0 & & & 19.8 \\
\hline & $\begin{array}{l}2380.54 \\
2303.54\end{array}$ & 41.23150 & 170.43920 & 1134.8 & & 251.01 & 329.2 \\
\hline
\end{tabular}


TABLE 3 - Continued

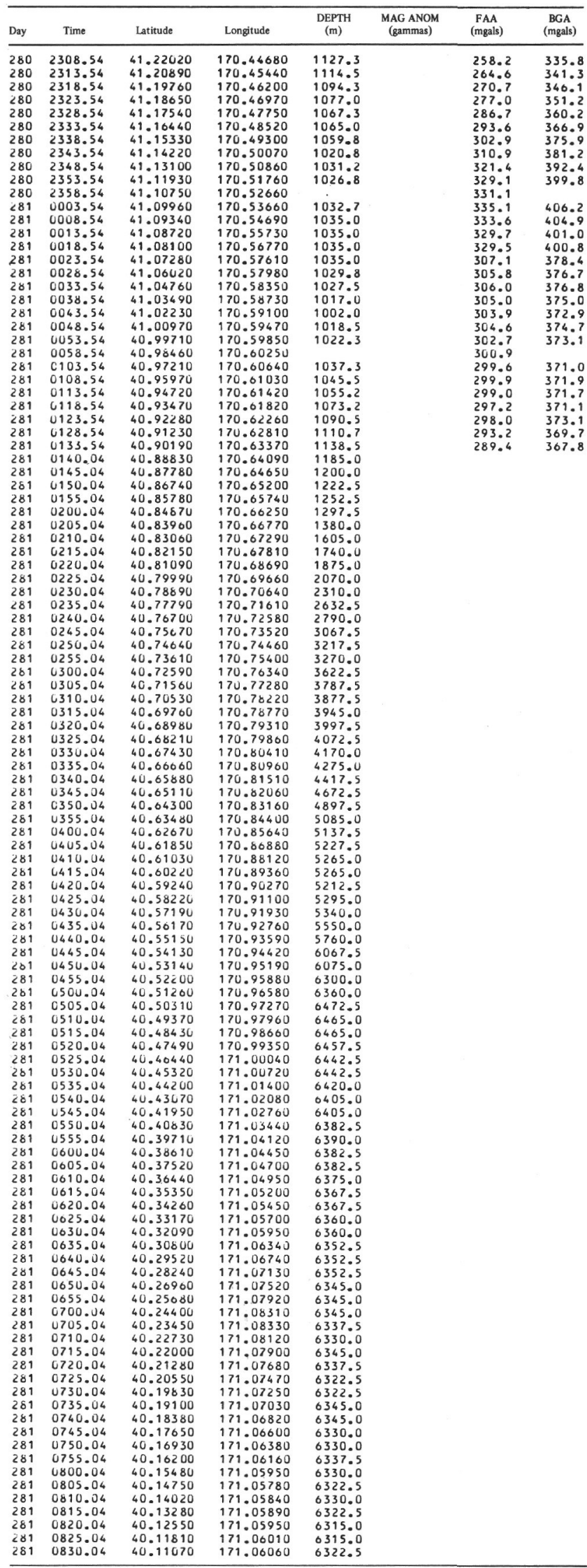

TABLE 3 - Continued

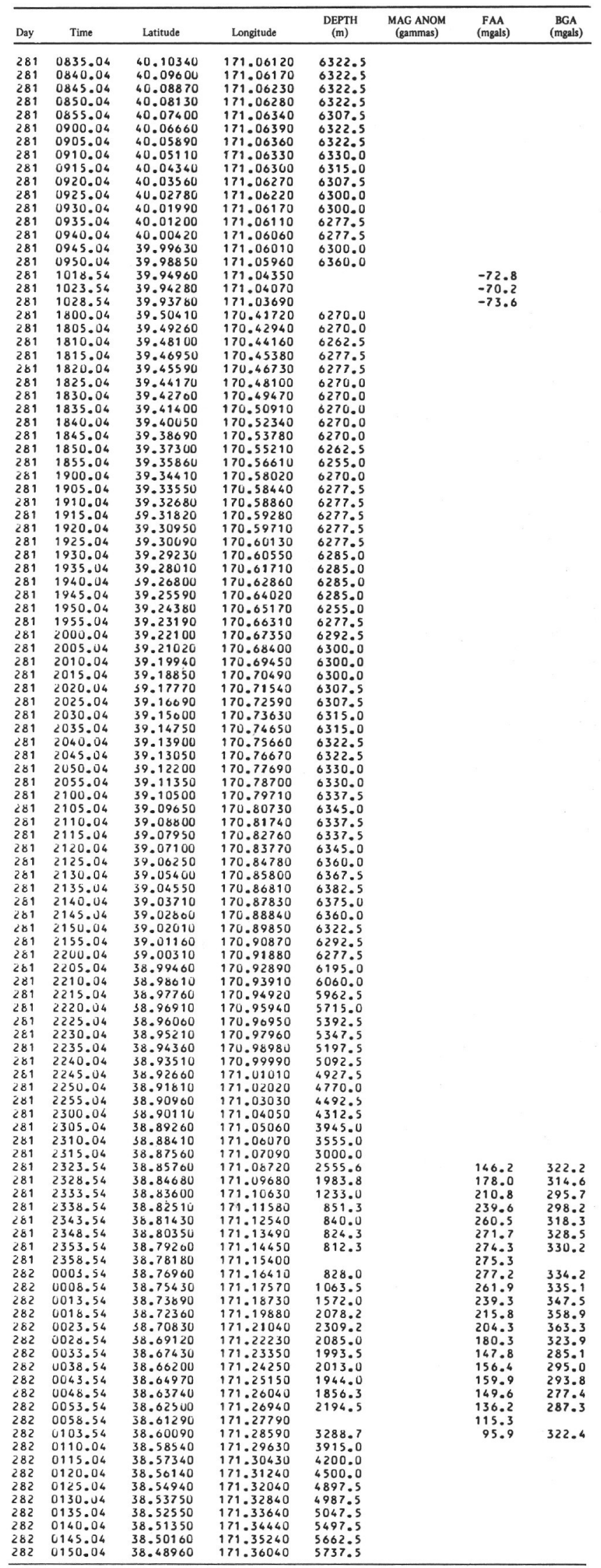


TABLE 3 - Continued

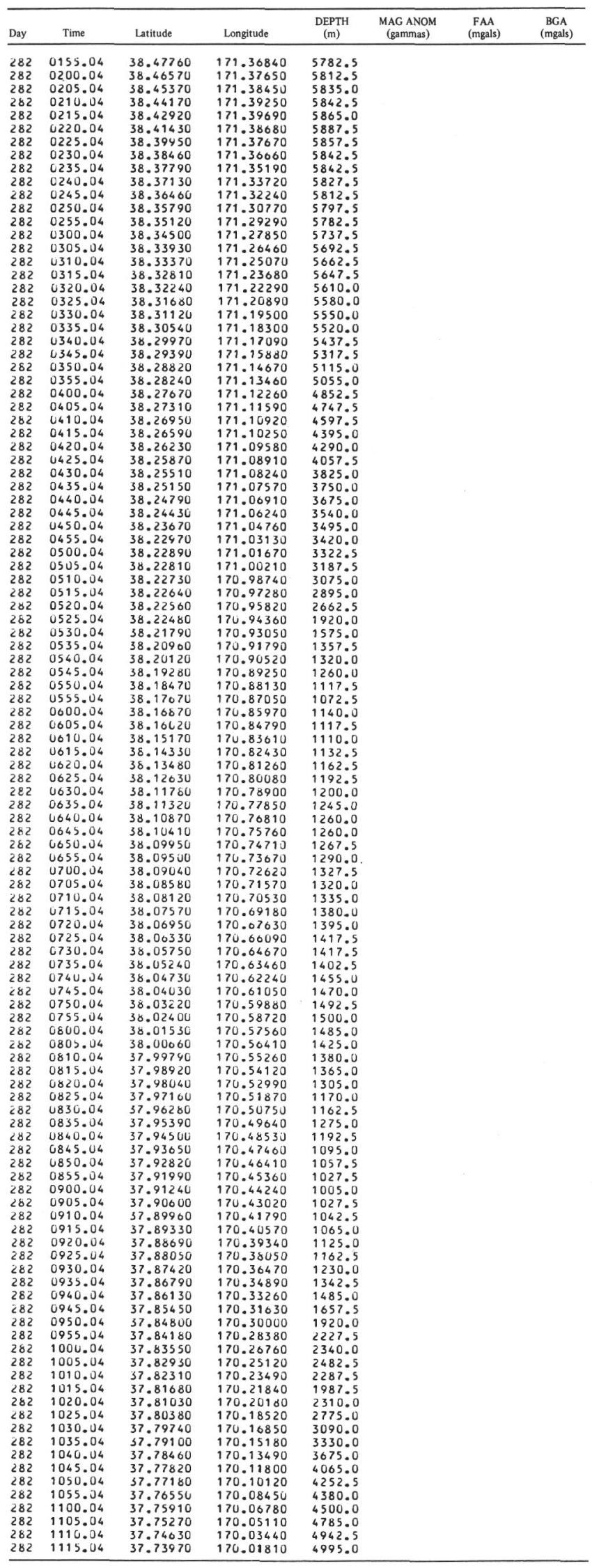

TABLE 3 - Continued

\begin{tabular}{|c|}
\hline$\underline{\text { Day }}$ \\
\hline 282 \\
\hline 282 \\
\hline 282 \\
\hline $\begin{array}{l}282 \\
282\end{array}$ \\
\hline 282 \\
\hline 282 \\
\hline 282 \\
\hline $\begin{array}{l}282 \\
282\end{array}$ \\
\hline 282 \\
\hline 282 \\
\hline 282 \\
\hline 282 \\
\hline 282 \\
\hline $\begin{array}{l}282 \\
282\end{array}$ \\
\hline 282 \\
\hline 282 \\
\hline 282 \\
\hline 282 \\
\hline $\begin{array}{l}282 \\
282\end{array}$ \\
\hline $\begin{array}{l}282 \\
282\end{array}$ \\
\hline 282 \\
\hline \\
\hline 282 \\
\hline 282 \\
\hline $\begin{array}{l}282 \\
282\end{array}$ \\
\hline $\begin{array}{l}282 \\
282\end{array}$ \\
\hline 282 \\
\hline 28 \\
\hline 282 \\
\hline 282 \\
\hline $\begin{array}{l}282 \\
282\end{array}$ \\
\hline 282 \\
\hline 282 \\
\hline 282 \\
\hline 282 \\
\hline 282 \\
\hline $\begin{array}{l}282 \\
282\end{array}$ \\
\hline $\begin{array}{l}282 \\
282\end{array}$ \\
\hline 282 \\
\hline 282 \\
\hline 282 \\
\hline 282 \\
\hline $\begin{array}{l}282 \\
282\end{array}$ \\
\hline 282 \\
\hline 282 \\
\hline 282 \\
\hline 282 \\
\hline 282 \\
\hline 282 \\
\hline $\begin{array}{c}682 \\
282\end{array}$ \\
\hline 282 \\
\hline 282 \\
\hline 282 \\
\hline 282 \\
\hline 282 \\
\hline $\begin{array}{l}82 \\
282\end{array}$ \\
\hline 282 \\
\hline 282 \\
\hline 282 \\
\hline 282 \\
\hline 282 \\
\hline 282 \\
\hline 282 \\
\hline 282 \\
\hline 282 \\
\hline 282 \\
\hline $\begin{array}{l}282 \\
282\end{array}$ \\
\hline 282 \\
\hline 282 \\
\hline 282 \\
\hline 282 \\
\hline 282 \\
\hline $\begin{array}{l}282 \\
282\end{array}$ \\
\hline 28 \\
\hline 282 \\
\hline 282 \\
\hline 282 \\
\hline 282 \\
\hline 282 \\
\hline 2 \\
\hline 28 \\
\hline 282 \\
\hline 282 \\
\hline i82 \\
\hline $\begin{array}{l}2828 \\
282\end{array}$ \\
\hline 282 \\
\hline \\
\hline 282 \\
\hline 282 \\
\hline 282 \\
\hline 282 \\
\hline 206 \\
\hline 282 \\
\hline 28 \\
\hline 282 \\
\hline 282 \\
\hline 282 \\
\hline
\end{tabular}


TABLE 3 - Continued

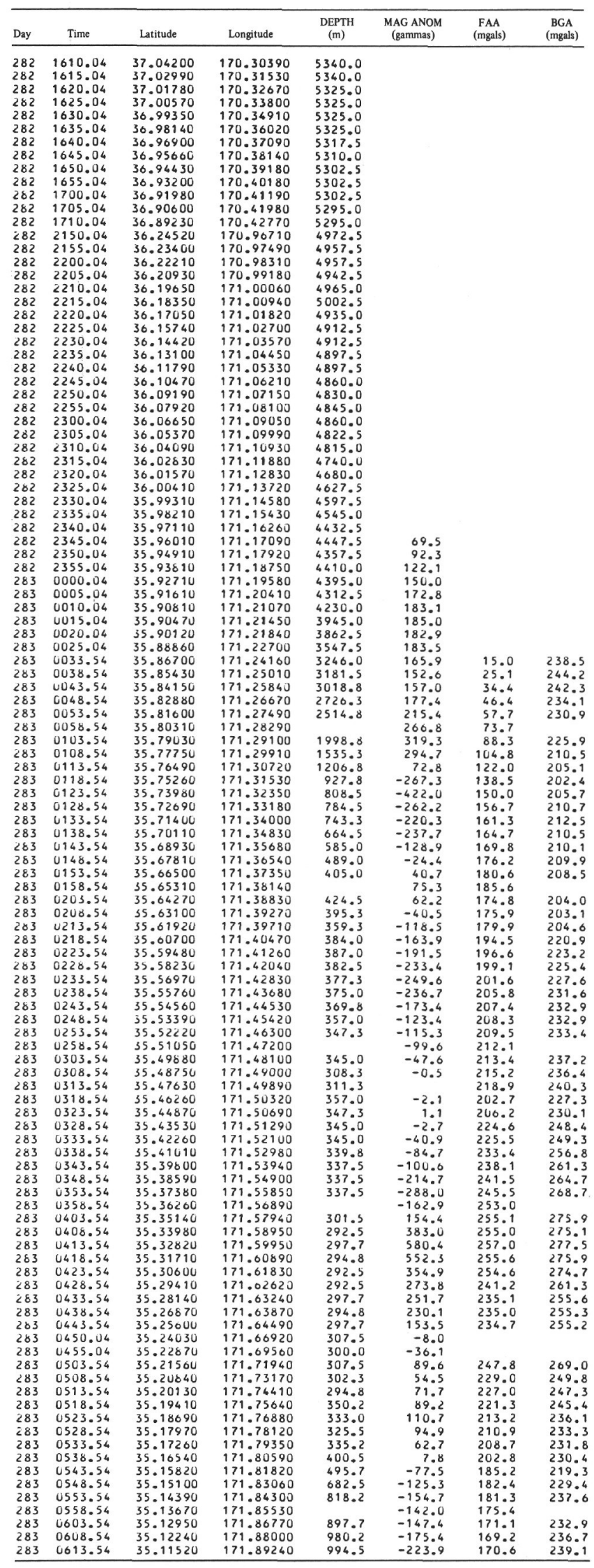

TABLE 3 - Continued

\begin{tabular}{|c|c|c|}
\hline Day & Time & Latitude \\
\hline $28 \pi$ & 0618.54 & 35.10800 \\
\hline $\begin{array}{l}283 \\
283\end{array}$ & $\begin{array}{l}0623.54 \\
0628.54\end{array}$ & $\begin{array}{l}35.10080 \\
35.09370\end{array}$ \\
\hline 283 & 0633.54 & 35.08650 \\
\hline $\begin{array}{l}283 \\
283\end{array}$ & $\begin{array}{l}0638.54 \\
0643.54\end{array}$ & $\begin{array}{l}35.07930 \\
35.07090\end{array}$ \\
\hline $\begin{array}{l}283 \\
283\end{array}$ & $\begin{array}{l}0648.54 \\
064.54\end{array}$ & $\begin{array}{l}35.07090 \\
35.05900\end{array}$ \\
\hline 283 & 0653.54 & \\
\hline 283 & 0700.04 & 35.05170 \\
\hline 283 & 0705.04 & 35.05550 \\
\hline 283 & 0710.04 & 35.05920 \\
\hline $\begin{array}{l}283 \\
283\end{array}$ & $\begin{array}{l}0718.54 \\
4723.54\end{array}$ & $\begin{array}{r}35.04930 \\
35.04050\end{array}$ \\
\hline $\begin{array}{l}283 \\
283\end{array}$ & $\begin{array}{l}0725.54 \\
0728.54\end{array}$ & 35.03160 \\
\hline 203 & 0733.54 & 35.02280 \\
\hline 283 & 0738.54 & 35.01400 \\
\hline 283 & 0743.54 & 35.00520 \\
\hline 263 & 0748.54 & 34.99040 \\
\hline $\begin{array}{l}283 \\
283\end{array}$ & $\begin{array}{l}0753.54 \\
0758.54\end{array}$ & $\begin{array}{l}34.98470 \\
34.96610\end{array}$ \\
\hline 283 & 0803.54 & 34.94700 \\
\hline & & \\
\hline 283 & 0813.54 & 34.91060 \\
\hline 283 & 0818.54 & 34.89670 \\
\hline 283 & 0823.54 & 34.88740 \\
\hline $\begin{array}{l}283 \\
283\end{array}$ & $\begin{array}{l}0888.54 \\
6833.54\end{array}$ & $\begin{array}{r}34.87820 \\
34.86890\end{array}$ \\
\hline & 0838.54 & 34.85970 \\
\hline (20) & 6843.54 & 34.85030 \\
\hline 283 & 0848.54 & 34.84060 \\
\hline 283 & 0853.54 & 34.83150 \\
\hline $\begin{array}{l}283 \\
283\end{array}$ & 0858.54 & 34.82360 \\
\hline 283 & $\begin{array}{l}9003.54 \\
0908.54\end{array}$ & $\begin{array}{l}34.81570 \\
36.80760\end{array}$ \\
\hline 283 & 0913.54 & 34.79920 \\
\hline 283 & 0918.54 & 34.79040 \\
\hline 283 & 0923.54 & 34.78150 \\
\hline 283 & 0928.54 & 34.77270 \\
\hline 283 & 0933.54 & 34.70230 \\
\hline 283 & $\begin{array}{l}438.54 \\
0943.54\end{array}$ & $\begin{array}{l}34.74810 \\
34.73390\end{array}$ \\
\hline 283 & 0948.54 & 34.72420 \\
\hline 283 & 0953.54 & 34.71500 \\
\hline 283 & 0958.54 & 34.70570 \\
\hline 283 & 1003.54 & 34.69650 \\
\hline $\begin{array}{l}283 \\
283\end{array}$ & 1008.54 & $\begin{array}{l}34.68740 \\
34.67850\end{array}$ \\
\hline $\begin{array}{l}283 \\
283\end{array}$ & $\begin{array}{l}113.54 \\
1018.54\end{array}$ & $\begin{array}{l}34.67850 \\
34.66950\end{array}$ \\
\hline 283 & 1023.54 & 34.66060 \\
\hline 283 & 1028.54 & 34.65140 \\
\hline 283 & 1033.54 & 34.64190 \\
\hline 283 & 1038.54 & 34.63250 \\
\hline $\begin{array}{l}283 \\
283\end{array}$ & $\begin{array}{l}1433.54 \\
1048.54\end{array}$ & $\begin{array}{l}34.62310 \\
34.61350\end{array}$ \\
\hline 283 & 1053.54 & 36.60380 \\
\hline $\begin{array}{l}283 \\
283\end{array}$ & 1058.54 & $\begin{array}{l}34.59430 \\
36.58506\end{array}$ \\
\hline 283 & $\begin{array}{l}1103.54 \\
1108.54\end{array}$ & $\begin{array}{l}34.58500 \\
34.57560\end{array}$ \\
\hline 283 & 1113.54 & 34.56030 \\
\hline 283 & 1118.54 & 34 : \\
\hline 283 & 1123.54 & 34.54770 \\
\hline 283 & 1128.54 & 34.53830 \\
\hline $\begin{array}{l}283 \\
283\end{array}$ & $\begin{array}{l}1133.54 \\
1138.54\end{array}$ & $\begin{array}{l}34.52900 \\
34.51990\end{array}$ \\
\hline 283 & $\begin{array}{l}1138.54 \\
1143.54\end{array}$ & $\begin{array}{l}54.51100 \\
34.51100\end{array}$ \\
\hline 283 & 1148.54 & 36.50220 \\
\hline 283 & 1153.54 & 34.49330 \\
\hline 283 & 1158.54 & 34.48440 \\
\hline 283 & 1203.54 & 34.47560 \\
\hline $\begin{array}{l}283 \\
283\end{array}$ & $\begin{array}{l}2128.54 \\
1213.54\end{array}$ & $\begin{array}{l}34.46060 \\
34.45700\end{array}$ \\
\hline 283 & 1218.54 & 34.44810 \\
\hline 283 & $\begin{array}{l}1223.54 \\
1328.54\end{array}$ & 34.43870 \\
\hline $\begin{array}{l}283 \\
283\end{array}$ & $\begin{array}{l}1228.54 \\
1233.54\end{array}$ & $\begin{array}{l}34.42950 \\
34.42040\end{array}$ \\
\hline & 1238.54 & 54.41120 \\
\hline 283 & 1243.54 & 34.40980 \\
\hline 28 & 1248.54 & 34.39220 \\
\hline 283 & 1253.54 & 34.38260 \\
\hline 283 & 125 & \\
\hline 28 & 1308.54 & 34.35 \\
\hline 283 & 131 & $u$ \\
\hline 283 & 1318.54 & 34.33530 \\
\hline $28>>-1$ & 1323.54 & 0 \\
\hline 283 & 13 & 3 \\
\hline & & 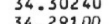 \\
\hline . & 4 & 0 \\
\hline $28-20-2$ & 13 & 0 \\
\hline $2 \varepsilon>-2-$ & & \\
\hline $2 \xi>>2-1$ & 13 & 0 \\
\hline 283 & 14 & 0 \\
\hline & & \\
\hline & & \\
\hline $28 \quad \longrightarrow$ & & \\
\hline 28 & & 0 \\
\hline 283 & 143 & 0 \\
\hline 283 & & 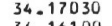 \\
\hline & & \\
\hline 28 & 1453.54 & 34.14 \\
\hline & & \\
\hline & & \\
\hline $28>>$ & & 0 \\
\hline & & \\
\hline 28 & & \\
\hline 283 & & 0 \\
\hline & & \\
\hline & 3.5 & 4.05270 \\
\hline 283 & 1543.54 & 34.04070 \\
\hline
\end{tabular}


TABLE 3 - Continued

TABLE 3 - Continued

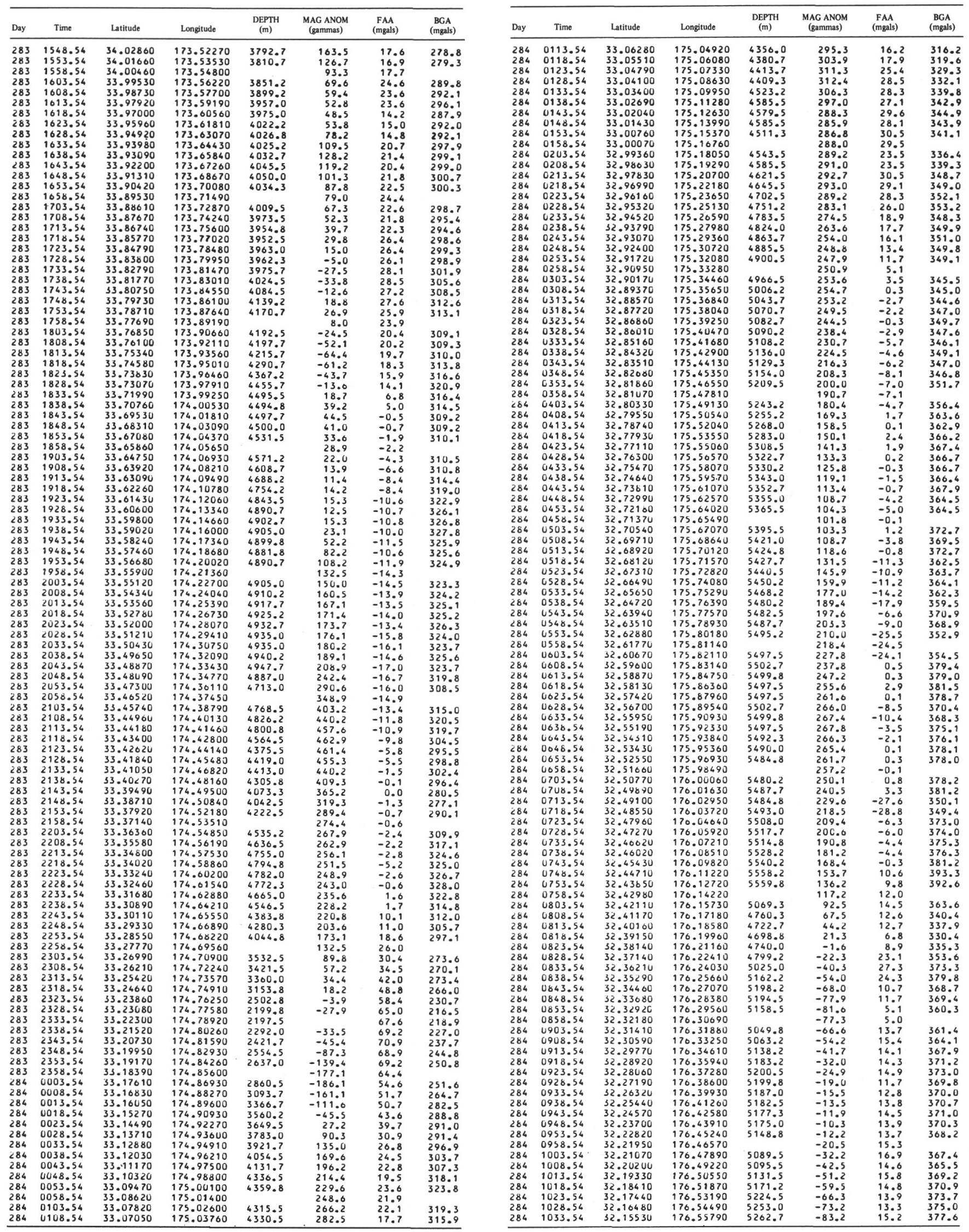


TABLE 3 - Continued

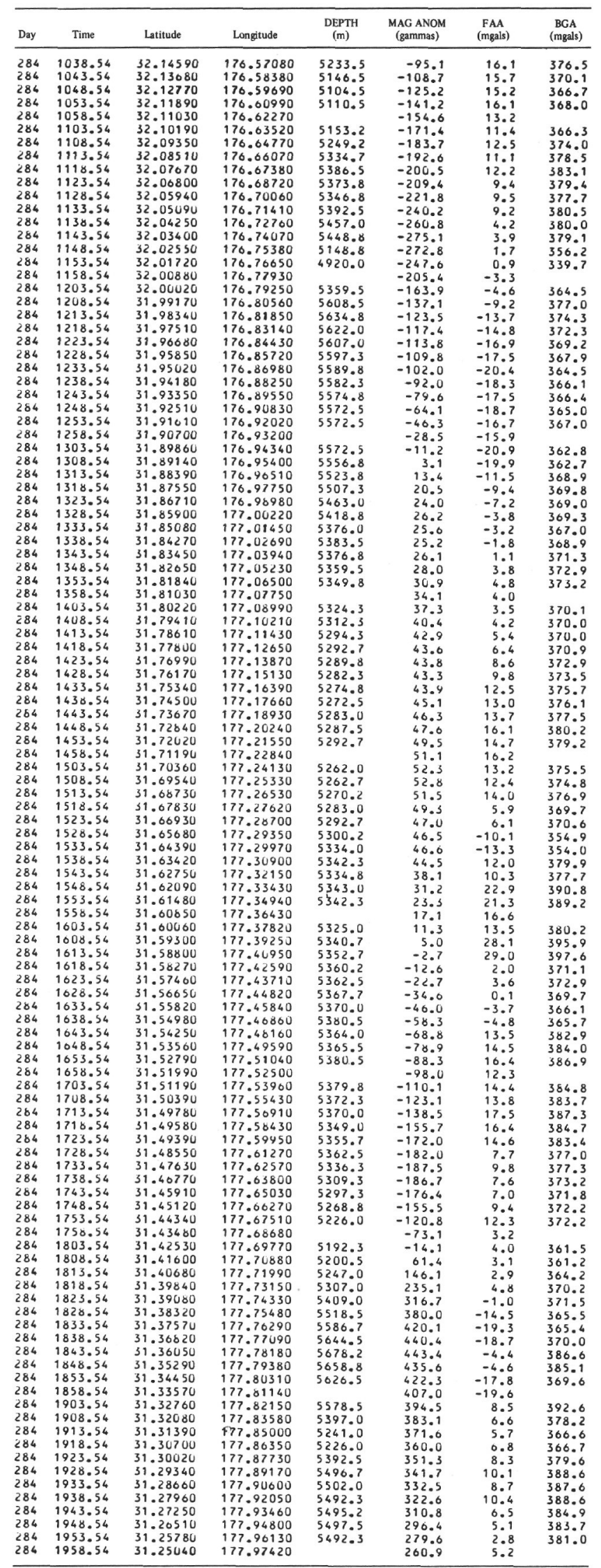

TABLE 3 - Continued

\begin{tabular}{|c|c|c|c|c|c|c|c|}
\hline Day & Time & Latitude & Longitude & $\begin{array}{c}\text { DEPTH } \\
(\mathrm{m})\end{array}$ & $\begin{array}{c}\text { MAG ANOM } \\
\text { (gammas) }\end{array}$ & $\begin{array}{c}\text { FAA } \\
\text { (mgals) }\end{array}$ & $\begin{array}{c}\text { BGA } \\
\text { (mgals) }\end{array}$ \\
\hline 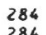 & 2003.54 & 31.24270 & 177.98700 & 5679.5 & 243.2 & 5.6 & 382.9 \\
\hline $\begin{array}{l}284 \\
284\end{array}$ & $\begin{array}{l}2008.56 \\
2013.54\end{array}$ & $\begin{array}{l}31.23600 \\
31.22520\end{array}$ & $\begin{array}{l}177.99960 \\
178.01220\end{array}$ & $\begin{array}{l}5506.5 \\
5488.5\end{array}$ & $\begin{array}{l}221.6 \\
197.6\end{array}$ & $\begin{array}{l}2.8 \\
1.1\end{array}$ & $\begin{array}{l}382.0 \\
379.0\end{array}$ \\
\hline 284 & 2018.54 & 31.21650 & 178.02480 & 5469.8 & 174.5 & 0.8 & 377.4 \\
\hline $\begin{array}{l}288 \\
284\end{array}$ & $\begin{array}{l}2023.54 \\
2028.56\end{array}$ & $\begin{array}{l}31.20780 \\
31.19900\end{array}$ & $\begin{array}{l}178.03740 \\
178.05010\end{array}$ & $\begin{array}{l}5472.7 \\
5501.2\end{array}$ & $\begin{array}{l}153.1 \\
136.5\end{array}$ & 1.8 & 378.7 \\
\hline 284 & $\begin{array}{l}2028.56 \\
2033.54\end{array}$ & $\begin{array}{l}31.19900 \\
31.19030\end{array}$ & $\begin{array}{l}178.505010 \\
178.06270\end{array}$ & $\begin{array}{l}5501.2 \\
5544.0\end{array}$ & $\begin{array}{l}136.5 \\
124.6\end{array}$ & $\begin{array}{l}4.5 \\
7.9\end{array}$ & $\begin{array}{l}383.3 \\
389.7\end{array}$ \\
\hline 284 & 2038.54 & 31.18160 & 178.07530 & 5557.5 & $\begin{array}{l}12.6 \\
113.1\end{array}$ & 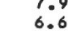 & $\begin{array}{l}389.7 \\
389.3\end{array}$ \\
\hline $\begin{array}{l}284 \\
284\end{array}$ & $\begin{array}{l}2043.54 \\
2048.54\end{array}$ & $\begin{array}{l}31.17290 \\
31.10410\end{array}$ & $\begin{array}{l}178.08800 \\
178.10060\end{array}$ & 5541.8 & 98.5 & 4.7 & 386.3 \\
\hline 284 & $\begin{array}{r}2048.54 \\
2053.54\end{array}$ & $\begin{array}{l}31.10410 \\
31.15540\end{array}$ & $\begin{array}{l}178.10060 \\
178.11310\end{array}$ & $\begin{array}{l}5519.3 \\
5507.3\end{array}$ & $\begin{array}{l}8.2 \\
76.8\end{array}$ & $\begin{array}{l}6.0 \\
5.4\end{array}$ & $\begin{array}{l}386.1 \\
384.6\end{array}$ \\
\hline $\begin{array}{l}284 \\
284\end{array}$ & 2058.54 & 31.14780 & 178.12610 & & 68.9 & 5.1 & \\
\hline $\begin{array}{l}284 \\
284\end{array}$ & $\begin{array}{l}2103.54 \\
2108.54\end{array}$ & 31.14140 & $\begin{array}{l}178.13950 \\
1788\end{array}$ & 5492.3 & 65.4 & & 382.0 \\
\hline 284 & $\begin{array}{l}2108.54 \\
2113.54\end{array}$ & $\begin{array}{l}31.13510 \\
31.12670\end{array}$ & $\begin{array}{r}178.15290 \\
178.10630\end{array}$ & 9 & $\begin{array}{l}62.9 \\
60.2\end{array}$ & 5. & 382.2 \\
\hline 284 & 2118.54 & 31.12230 & 178.17960 & 5447.3 & 55.3 & 4.5 & $\begin{array}{l}380.9 \\
379.6\end{array}$ \\
\hline 284 & 2123.54 & 31.11580 & 178.19290 & 5618.8 & 51.0 & 4.5 & 377.6 \\
\hline $\begin{array}{l}284 \\
284\end{array}$ & $\begin{array}{l}2128.54 \\
2133.54\end{array}$ & $\begin{array}{r}31.10930 \\
31.10270\end{array}$ & 178.20610 & 5602.3 & 45.9 & 2.7 & 374.7 \\
\hline 284 & $\begin{array}{l}2133.54 \\
2138.54\end{array}$ & $\begin{array}{l}31.10270 \\
31.09620\end{array}$ & $\begin{array}{l}178.221940 \\
178.23240\end{array}$ & $\begin{array}{l}5426.2 \\
5458.5\end{array}$ & $\begin{array}{l}36.7 \\
24.6\end{array}$ & 5.6 & 379.3 \\
\hline 284 & 2143.54 & 31.08980 & 178.24540 & 5462.3 & 11.1 & $\begin{array}{l}5.1 \\
5.8\end{array}$ & $\begin{array}{l}381.0 \\
381.9\end{array}$ \\
\hline 284 & 2148.54 & 31.08360 & 178.25830 & 5469.5 & -4.0 & 5.6 & $\begin{array}{l}381.9 \\
380.9\end{array}$ \\
\hline $\begin{array}{l}284 \\
284\end{array}$ & $\begin{array}{l}2153.54 \\
2158.54\end{array}$ & $\begin{array}{l}31.07710 \\
31.07670\end{array}$ & $\begin{array}{r}178.27120 \\
178.28410\end{array}$ & 5424.0 & $\begin{array}{l}-18.4 \\
-29.3\end{array}$ & $\begin{array}{l}4.4 \\
6.8\end{array}$ & 377.9 \\
\hline 284 & 2203.54 & 31.06420 & 178.29700 & 5412.7 & $\begin{array}{l}-290.5 \\
-37.7\end{array}$ & $\begin{array}{l}0.8 \\
7.3\end{array}$ & 380.0 \\
\hline 284 & 2208.54 & 31.05780 & 178.30990 & 5430.7 & -46.2 & & $\begin{array}{l}380.0 \\
377.0\end{array}$ \\
\hline $\begin{array}{l}284 \\
284\end{array}$ & 2213.54 & $\begin{array}{l}31.05150 \\
31.06502\end{array}$ & 178.32230 & & -55.0 & & 381.0 \\
\hline 284 & $\begin{array}{l}2218.54 \\
2223.54\end{array}$ & 31.03870 & $\begin{array}{r}178.333480 \\
178.34730\end{array}$ & $\begin{array}{l}5439.0 \\
5435.2\end{array}$ & $\begin{array}{l}-65.8 \\
-75\end{array}$ & & 381.4 \\
\hline 284 & 2228.54 & 31.03210 & 178.35980 & 5432.3 & $\begin{array}{l}-1,5.4 \\
-85.5\end{array}$ & & 376.6 \\
\hline 284 & 2233.54 & 31.02540 & 178.37230 & 5382.8 & -92.6 & $\begin{array}{l}2 \\
0\end{array}$ & $\begin{array}{l}376.7 \\
371.4\end{array}$ \\
\hline 284 & 2238.54 & 31.01860 & 178.38480 & 5367.7 & -99.6 & 4.4 & 374.0 \\
\hline $\begin{array}{l}284 \\
284\end{array}$ & $\begin{array}{l}2263.54 \\
2248.54\end{array}$ & $\begin{array}{l}31.01160 \\
31.00600\end{array}$ & $\begin{array}{l}178.39710 \\
178.40960\end{array}$ & $\begin{array}{l}5396.2 \\
5439.0\end{array}$ & $\begin{array}{l}-103.0 \\
-104.6\end{array}$ & $\begin{array}{l}4.2 \\
5.0\end{array}$ & 375.8 \\
\hline 284 & 2253.54 & 30.99750 & 178.42200 & 5426.3 & $\begin{array}{l}-104.6 \\
-104.0\end{array}$ & 40 & $\begin{array}{l}379.5 \\
378.1\end{array}$ \\
\hline $\begin{array}{l}284 \\
284\end{array}$ & $\begin{array}{l}2258.56 \\
230354\end{array}$ & $\begin{array}{l}30.99050 \\
30.98400\end{array}$ & $\begin{array}{l}178.43420 \\
178.44300\end{array}$ & 5395.5 & $\begin{array}{l}-98.6 \\
-89.0\end{array}$ & -10.1 & \\
\hline 284 & 2308.54 & 30.97870 & 178.45190 & $5421: 0$ & $\begin{array}{l}-89.0 \\
-79.7\end{array}$ & & $\begin{array}{l}370.8 \\
370.1\end{array}$ \\
\hline 284 & 2313.54 & 30.97280 & 178.46070 & 5445.7 & -68.5 & 3.1 & $\begin{array}{l}376.1 \\
378.1\end{array}$ \\
\hline $\begin{array}{l}284 \\
284\end{array}$ & $\begin{array}{l}2318.54 \\
2323.54\end{array}$ & $\begin{array}{l}30.96710 \\
30.96100\end{array}$ & $\begin{array}{l}178.46930 \\
178.47770\end{array}$ & $\begin{array}{l}5431.5 \\
5391.0\end{array}$ & $\begin{array}{l}-55.1 \\
-39.7\end{array}$ & $\begin{array}{l}-1.5 \\
-0.9\end{array}$ & $\begin{array}{l}372.5 \\
370.3\end{array}$ \\
\hline 284 & 2328.54 & 30.95600 & 178.48600 & 5388.0 & -23.0 & $\begin{array}{r}-0.9 \\
2.7\end{array}$ & $\begin{array}{l}370.3 \\
373.7\end{array}$ \\
\hline $\begin{array}{l}284 \\
284\end{array}$ & $\begin{array}{l}2333.54 \\
2338.54\end{array}$ & $\begin{array}{l}30.95040 \\
30.94490\end{array}$ & $\begin{array}{l}178.49450 \\
178.50300\end{array}$ & $\begin{array}{l}5408.2 \\
5420.2\end{array}$ & -6.7 & 1.6 & 374.0 \\
\hline 284 & 2343.54 & 30.93950 & 178.51140 & $\begin{array}{l}5420.2 \\
5433.0\end{array}$ & $\begin{array}{l}10.0 \\
28.3\end{array}$ & $\begin{array}{l}1.64 \\
2.2\end{array}$ & $\begin{array}{l}374.6 \\
376.3\end{array}$ \\
\hline & 2348.54 & 30.93430 & 178.51980 & 5427.0 & 47.9 & -0.5 & $\begin{array}{l}376.3 \\
373.2\end{array}$ \\
\hline $\begin{array}{l}284 \\
284\end{array}$ & $\begin{array}{l}2353.54 \\
2358.54\end{array}$ & $\begin{array}{l}30.92880 \\
30.92300\end{array}$ & $\begin{array}{l}178.52840 \\
178.53740\end{array}$ & 5438.2 & $\begin{array}{l}67.1 \\
85.5\end{array}$ & 0.5 & 375.0 \\
\hline 285 & 0003.54 & 30.91720 & 178.54640 & 5452.5 & $\begin{array}{l}85.5 \\
104.3\end{array}$ & & 376.6 \\
\hline 285 & 0008.54 & 30.90920 & 178.5 & 0 & 120.6 & & \\
\hline $\begin{array}{l}285 \\
285\end{array}$ & 0013.54 & 30.90110 & 178.50040 & 5467.5 & 136.4 & -9.0 & $\begin{array}{l}300.3 \\
367.5\end{array}$ \\
\hline 285 & $\begin{array}{r}0078.54 \\
0023.54\end{array}$ & $\begin{array}{l}50.893520 \\
30.88790\end{array}$ & $\begin{array}{l}178.56760 \\
178.57760\end{array}$ & $\begin{array}{l}5462.3 \\
5465.2\end{array}$ & 151.6 & $\cdots$ & 380.5 \\
\hline 285 & 0028.54 & 30.88260 & 178.58760 & $\begin{array}{l}5462.3 \\
5462.3\end{array}$ & 178.1 & $\begin{array}{l}2.0 \\
6.3\end{array}$ & 378.3 \\
\hline$<85$ & 0033.54 & 30.87750 & 178.59780 & 5433.8 & 190.5 & 4.3 & 378.5 \\
\hline $\begin{array}{l}285 \\
285\end{array}$ & 0038.54 & $\begin{array}{l}30.87240 \\
30.8670\end{array}$ & 178.60800 & 5438.2 & 203.0 & 4.6 & 379.1 \\
\hline $\begin{array}{l}285 \\
285\end{array}$ & $\begin{array}{l}0033.54 \\
0048.56\end{array}$ & $\begin{array}{l}\begin{array}{r}30.86720 \\
30.86190\end{array} \\
30.81\end{array}$ & $\begin{array}{l}178.01830 \\
178.62850\end{array}$ & $\begin{array}{l}5400.7 \\
5459.8\end{array}$ & $\begin{array}{l}215.1 \\
226.9\end{array}$ & $\begin{array}{l}4.8 \\
3.9\end{array}$ & $\begin{array}{l}380.8 \\
379.3\end{array}$ \\
\hline 285 & 0053.54 & 30.85670 & 178.63880 & 5450.2 & 238.7 & 2.4 & 377.7 \\
\hline $\begin{array}{l}285 \\
285\end{array}$ & $\begin{array}{l}0058.54 \\
0103.54\end{array}$ & $\begin{array}{l}30.85130 \\
30.84590\end{array}$ & $\begin{array}{l}178.04950 \\
178.06020\end{array}$ & 5434.5 & $\begin{array}{l}249.1 \\
2575\end{array}$ & 3.7 & \\
\hline 285 & 0108.54 & 30.84040 & 178.07110 & 5 & 264.0 & $\begin{array}{l}0.04 \\
6.4\end{array}$ & $\begin{array}{l}380.9 \\
381.0\end{array}$ \\
\hline $\begin{array}{l}285 \\
285\end{array}$ & 0113.54 & 30.83460 & 178.68220 & 545 & 59.2 & 4.5 & 379.8 \\
\hline $\begin{array}{l}285 \\
285\end{array}$ & $\begin{array}{l}0118.54 \\
0123.54\end{array}$ & $\begin{array}{l}30.82900 \\
30.82340\end{array}$ & $\begin{array}{l}178.69330 \\
178.70430\end{array}$ & & $\begin{array}{l}269.2 \\
265.1\end{array}$ & $\begin{array}{l}4.8 \\
4.5\end{array}$ & 379.9 \\
\hline 285 & 0128.54 & 30.81770 & 178.71540 & 5445.0 & 258.0 & 3.7 & $\begin{array}{l}379.6 \\
378.6\end{array}$ \\
\hline $\begin{array}{l}\dot{i} 85 \\
285\end{array}$ & $\begin{array}{l}0133.54 \\
413854\end{array}$ & $\begin{array}{l}30.81160 \\
30.80590\end{array}$ & $\begin{array}{l}178.72640 \\
178.73730\end{array}$ & $\begin{array}{l}5434.5 \\
5435.2\end{array}$ & $\begin{array}{l}250.2 \\
234.2\end{array}$ & 2.4 & 370.6 \\
\hline $\begin{array}{l}285 \\
285\end{array}$ & $\begin{array}{l}0138.54 \\
0143.54\end{array}$ & $\begin{array}{r}30.80590 \\
30.79990\end{array}$ & $\begin{array}{l}178.73730 \\
178.74820\end{array}$ & $\begin{array}{l}5435.2 \\
5437.5\end{array}$ & $\begin{array}{l}234.2 \\
215.3\end{array}$ & $\begin{array}{l}3.2 \\
5.0\end{array}$ & $\begin{array}{l}377.5 \\
379.4\end{array}$ \\
\hline 285 & 0148.54 & 30.79360 & 178.75880 & 5432.3 & 195.7 & 1.3 & $\begin{array}{l}379.4 \\
375.4\end{array}$ \\
\hline $\begin{array}{l}285 \\
285\end{array}$ & 0153.54 & 30.78720 & 930 & 5430.0 & 170 & & 375.0 \\
\hline $\begin{array}{l}285 \\
285\end{array}$ & $\begin{array}{l}0158.54 \\
0203.54\end{array}$ & $\begin{array}{l}30.78090 \\
30.77450\end{array}$ & $\begin{array}{l}178.77990 \\
178\end{array}$ & 5435.2 & & 1 & \\
\hline 285 & $\begin{array}{r}0203.54 \\
0208.54\end{array}$ & 30.76820 & 178.80100 & & $\begin{array}{l}129.7 \\
129.7\end{array}$ & -2.0 & 372.1 \\
\hline 285 & 0213.54 & 30.76210 & 178.81110 & 5435.2 & 117.2 & 3.1 & 377.4 \\
\hline 285 & 0218.54 & 30.75610 & 178.82130 & 5437.5 & 106.7 & 1.4 & 375.8 \\
\hline $\begin{array}{l}285 \\
285 \\
285\end{array}$ & 0223.54 & 30 & & & $\begin{array}{l}97.5 \\
87.9\end{array}$ & 0.8 & 5.2 \\
\hline $\begin{array}{l}285 \\
285\end{array}$ & $\begin{array}{l}0288.54 \\
0233.54\end{array}$ & $\begin{array}{l}\begin{array}{r}30.74380 \\
30.73760\end{array} \\
3\end{array}$ & $\begin{array}{r}178.84180 \\
178.85230\end{array}$ & $\begin{array}{l}5437.5 \\
5421.8\end{array}$ & $\begin{array}{l}87.9 \\
78.0\end{array}$ & $\begin{array}{r}-0.2 \\
2.2\end{array}$ & $\begin{array}{l}374.2 \\
375.5\end{array}$ \\
\hline 285 & 0238.54 & 30.73150 & 178.8 & 542 & 68 & 1.4 & 374.6 \\
\hline $\begin{array}{l}285 \\
285\end{array}$ & $\begin{array}{l}0263.54 \\
0238.54\end{array}$ & $\begin{array}{l}30.72540 \\
30.71940\end{array}$ & $\begin{array}{l}178.87360 \\
178.88400\end{array}$ & $\begin{array}{l}5422.5 \\
5422.5\end{array}$ & $\begin{array}{l}60.3 \\
52.7\end{array}$ & 3.4 & 376.8 \\
\hline 265 & 0253.54 & 30.71350 & $\begin{array}{l}178.88400 \\
178.89440\end{array}$ & $\begin{array}{l}5422.5 \\
5422.5\end{array}$ & $\begin{array}{l}52.7 \\
44.7\end{array}$ & $\begin{array}{l}4.1 \\
1.8\end{array}$ & $\begin{array}{l}377.5 \\
375.2\end{array}$ \\
\hline $\begin{array}{l}285 \\
285\end{array}$ & $\begin{array}{l}0258.54 \\
0303.54\end{array}$ & $\begin{array}{l}30.70740 \\
30.70130\end{array}$ & $\begin{array}{r}178.90480 \\
178.91510\end{array}$ & & $\begin{array}{l}36 \\
30\end{array}$ & 1.7 & \\
\hline 285 & 0308.54 & 30 & $\begin{array}{l}178.91510 \\
178.92550\end{array}$ & & $\begin{array}{l}34.2 \\
24.3\end{array}$ & 50 & $\begin{array}{l}375.0 \\
376.9\end{array}$ \\
\hline 285 & 0313.54 & 30 & 1 & & 15 & 6. & 376.7 \\
\hline $\begin{array}{l}285 \\
285\end{array}$ & $\begin{array}{l}0318.54 \\
0323.54\end{array}$ & $\begin{array}{l}30.683340 \\
30.67680\end{array}$ & 178.94 & & $\begin{array}{r}4.3 \\
-7.5\end{array}$ & 7 & 376.3 \\
\hline $\begin{array}{l}285 \\
285\end{array}$ & $\begin{array}{l}0328.54 \\
03284\end{array}$ & $\begin{array}{l}30.67800 \\
30.66940\end{array}$ & 178.95 & $\begin{array}{l}4840.5 \\
4515.0\end{array}$ & $\begin{array}{l}-7.5 \\
-17.8\end{array}$ & $\begin{array}{l}7.8 \\
8.3\end{array}$ & $\begin{array}{l}41.1 \\
19.2\end{array}$ \\
\hline $\begin{array}{l}285 \\
285\end{array}$ & 03 & & & & $\begin{array}{l}-23.4 \\
-32.3\end{array}$ & 8.6 & \\
\hline $\begin{array}{l}285 \\
285\end{array}$ & $\begin{array}{l}0338.54 \\
0333.54\end{array}$ & $\begin{array}{l}30.65030 \\
30.6460\end{array}$ & $\begin{array}{l}178.98980 \\
179.00020\end{array}$ & $\begin{array}{l}4471.5 \\
47370\end{array}$ & $\begin{array}{l}-24.3 \\
-21.9\end{array}$ & $\begin{array}{l}7.99 \\
8.2\end{array}$ & 315.8 \\
\hline 285 & $\begin{array}{l}0345.54 \\
0348.54\end{array}$ & $\begin{array}{l}30.64 \\
30.64\end{array}$ & 179.01040 & $\begin{array}{l}4137.0 \\
4944.7\end{array}$ & $\begin{array}{l}-21.9 \\
-16.6\end{array}$ & 8.6 & $\begin{array}{l}334.4 \\
343.8\end{array}$ \\
\hline 203 & 0353.54 & 30.63600 & 179.02030 & 5208.0 & -8 & 2.3 & 360.9 \\
\hline 28 & 0358.54 & 30.63020 & 179003040 & & $\begin{array}{l}-2 \\
-0\end{array}$ & 7.0 & \\
\hline $\begin{array}{l}285 \\
285\end{array}$ & $\begin{array}{l}04 \\
04\end{array}$ & & & & $\begin{array}{l}-0 \\
-0\end{array}$ & 0.0 & 4 \\
\hline 285 & $\begin{array}{r}0413.54 \\
0454\end{array}$ & & 0 & & & 27.8 & 350.1 \\
\hline 265 & 0418.54 & 30 & 179. & & -3 & -5.6 & 71.7 \\
\hline 28 & 0423. & 30.59 & 1 & & & 9.1 & \\
\hline 185 & $\begin{array}{l}0428.54 \\
0433354\end{array}$ & $\begin{array}{l}30.57800 \\
30.56034\end{array}$ & 179. & 54 & -1 & $\begin{array}{l}-6.6 \\
-8 . ?\end{array}$ & :0 \\
\hline & $\begin{array}{l}04338.54 \\
0438.54\end{array}$ & $\begin{array}{l}50.560000 \\
30.55390\end{array}$ & 0 & & $\begin{array}{l}-29.9 \\
-42.2\end{array}$ & 2 & 306.5 \\
\hline & .54 & 3 & 179.06260 & & $\begin{array}{l}-4.25 .6 \\
-5.54\end{array}$ & . & $\begin{array}{l}300.9 \\
367.0\end{array}$ \\
\hline & & & & & -70 . & & 365.5 \\
\hline 28 & 0453.54 & & 1 & 543 & $-80<>0$ & 0.7 & 374.8 \\
\hline c & & & & & & 1.6 & \\
\hline 285 & 0508.54 & 30. & 179.06700 & 54 & -1 & 3. & 375.5 \\
\hline 285 & 0513.54 & 30.47 & 179.06920 & 5400.0 & -16 & 5. & 377.2 \\
\hline $\begin{array}{l}285 \\
285\end{array}$ & $\begin{array}{l}0518.54 \\
0523.54\end{array}$ & $\begin{array}{l}30.46260 \\
30.45670\end{array}$ & $\begin{array}{r}179.07110 \\
179.07060\end{array}$ & $\begin{array}{l}5405.2 \\
5412.7\end{array}$ & -158.1 & -1.7 & $\begin{array}{l}367.6 \\
371: 0\end{array}$ \\
\hline
\end{tabular}


TABLE 3 - Continued

\begin{tabular}{|c|c|c|c|c|c|c|c|}
\hline ay & Time & Latitude & Longitude & $\underset{(\mathrm{m})}{\text { DEPTH }}$ & 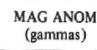 & $\left.\begin{array}{c}\mathrm{FAA} \\
(\text { mgalls) }\end{array}\right)$ & 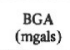 \\
\hline 285 & $\begin{array}{l}5528.54 \\
0533.54\end{array}$ & $\begin{array}{l}30.43880 \\
30.62060\end{array}$ & $\begin{array}{l}179.07010 \\
179.00990\end{array}$ & $\begin{array}{l}5399.3 \\
5371.5\end{array}$ & -160.4 & $\begin{array}{r}-2.7 \\
0.0\end{array}$ & $\begin{array}{l}369.1 \\
369.9\end{array}$ \\
\hline & $\begin{array}{l}0538.54 \\
0534.54 \\
0.54\end{array}$ & $\begin{array}{l}30.41450 \\
30.40220\end{array}$ & $\begin{array}{l}179.00990 \\
179.00980\end{array}$ & 53520 & $\begin{array}{r}-145.0 \\
-1427 \\
-127\end{array}$ & $\begin{array}{r}3.4 \\
-1.4\end{array}$ & 19 \\
\hline & $\begin{array}{l}0548.54 \\
0586.54\end{array}$ & $\begin{array}{l}30.40220 \\
30.38900\end{array}$ & $\begin{array}{r}179.000980 \\
77906900\end{array}$ & $\begin{array}{l}5362.3 \\
5329.5\end{array}$ & $\begin{array}{l}-1272.4 \\
-102.0 \\
\end{array}$ & $\begin{array}{r}-1.6 \\
3.4\end{array}$ & \\
\hline 28 & $\begin{array}{l}\begin{array}{l}55533.54 \\
0558.56\end{array} \\
058.5\end{array}$ & $\begin{array}{l}30.37710 \\
30.36520\end{array}$ & $\begin{array}{l}779-009090 \\
179000040\end{array}$ & 5319.8 & $\begin{array}{r}-672.3 \\
-22.8\end{array}$ & $\because 4$ & $3700^{\circ}$ \\
\hline & $\begin{array}{r}0.003 .54 \\
0008.54\end{array}$ & $\begin{array}{l}30.35640 \\
30.34280\end{array}$ & $\begin{array}{l}179.00900 \\
179000990\end{array}$ & $\begin{array}{l}5258.3 \\
5237.3\end{array}$ & $\begin{array}{r}29.3 \\
99.3 \\
91.3\end{array}$ & 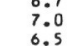 & $\begin{array}{l}36991 \\
36701\end{array}$ \\
\hline & $\begin{array}{l}0.51354 \\
0018554\end{array}$ & $\begin{array}{l}30.33120 \\
30.31960\end{array}$ & 179.009090 & 5203.5 & & & 365.2 \\
\hline & 0062.54 & $\begin{array}{l}30.3000 \\
330000\end{array}$ & $\begin{array}{r}179.008800 \\
1790\end{array}$ & $\begin{array}{l}5125.5 \\
5125\end{array}$ & 年 292.3 & $\begin{array}{l}9.4 \\
9.7\end{array}$ & $\begin{array}{l}365.7 \\
362.0\end{array}$ \\
\hline . & $\begin{array}{l}06288.54 \\
0633.54\end{array}$ & $\begin{array}{l}30.29640 \\
30.28480\end{array}$ & $\begin{array}{l}179.008000 \\
179.06750\end{array}$ & $\begin{array}{l}5102.3 \\
5089.5 \\
5\end{array}$ & $\begin{array}{r}\begin{array}{r}550.9 \\
392.2\end{array} \\
392\end{array}$ & $\begin{array}{r}9.5 \\
10.5\end{array}$ & $\begin{array}{l}360.8 \\
336.0\end{array}$ \\
\hline & 0604.54 & & $\begin{array}{l}799.007000 \\
179.06050\end{array}$ & $\begin{array}{l}5090.2 \\
5092.5\end{array}$ & $\begin{array}{l}149.0 \\
425.0\end{array}$ & 12 & \\
\hline & $\begin{array}{l}8.54 \\
8.54\end{array}$ & 30.25030 & 179.006560 & 5097.7 & 412.3 & $\begin{array}{l}11.7 \\
10.2\end{array}$ & $\begin{array}{l}362.4 \\
361.2\end{array}$ \\
\hline & 0658. & $\begin{array}{l}30.23000 \\
30.22880\end{array}$ & $\begin{array}{r}179.004000 \\
179000330\end{array}$ & 5063.3 & $\begin{array}{l}\begin{array}{r}388.1 \\
359.8\end{array} \\
359\end{array}$ & $\begin{array}{l}10.9 \\
19: 0\end{array}$ & 359.6 \\
\hline & $\begin{array}{l}0 \\
0708.54 \\
0.54\end{array}$ & $\begin{array}{l}30.21810 \\
30.20730\end{array}$ & $\begin{array}{l}179.062200 \\
179.06110\end{array}$ & $\begin{array}{l}\begin{array}{r}5056.5 \\
4963.5\end{array} \\
4.5\end{array}$ & $\begin{array}{l}336.4 \\
310.6\end{array}$ & $\begin{array}{l}10.0 \\
10.8\end{array}$ & $\begin{array}{r}358.2 \\
335.6\end{array}$ \\
\hline & $\begin{array}{l}3.54 \\
8.54\end{array}$ & $\begin{array}{l}30.19640 \\
30.18490\end{array}$ & $\begin{array}{l}179.06030 \\
179000000\end{array}$ & $\begin{array}{l}4969.5 \\
4971.8\end{array}$ & $\begin{array}{r}280.6 \\
254.2\end{array}$ & 18.2 & 330.6 \\
\hline & 0723.54 & 30.17330 & 179.06050 & 4970.2 & 230.9 & $\begin{array}{l}10.0 \\
10.0\end{array}$ & \\
\hline & 0180.34 & 30.15090 & 179.06260 & $\begin{aligned} 5009.2 \\
4988.3\end{aligned}$ & $\begin{array}{l}210.2 \\
189.7\end{array}$ & 22.3 & $367:$ \\
\hline & 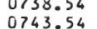 & $\begin{array}{l}30.13980 \\
30.182870\end{array}$ & $\begin{array}{l}179.06390 \\
179\end{array}$ & $\begin{array}{r}4977.7 \\
5027.2\end{array}$ & 167.5 & $\begin{array}{l}23.8 \\
24.4\end{array}$ & $\begin{array}{l}3667.3 \\
367.2\end{array}$ \\
\hline & & 30.11700 & & 500 & & & \\
\hline & $\begin{array}{l}\begin{array}{r}0753.54 \\
0758.554\end{array}\end{array}$ & $\begin{array}{l}30.10050 \\
30.09590\end{array}$ & $\begin{array}{l}179.068800 \\
179\end{array}$ & 4948.5 & $\begin{array}{c}100.0 \\
87.9 \\
8\end{array}$ & $\begin{array}{l}25.9 \\
25.6\end{array}$ & 360. \\
\hline & $\begin{array}{l}\begin{array}{l}08030.54 \\
0808.54\end{array} \\
0.54\end{array}$ & $\begin{array}{l}30.08530 \\
30.07440\end{array}$ & $\begin{array}{r}179.06990 \\
179 \\
0.07020\end{array}$ & $\begin{array}{l}4911.0 \\
48699^{\circ}\end{array}$ & $\begin{array}{l}73.6 \\
59.1\end{array}$ & $\begin{array}{l}25 . \\
21 .\end{array}$ & $\begin{array}{l}363 \\
587\end{array}$ \\
\hline & $\begin{array}{l}0811.54 \\
0818.54\end{array}$ & $\begin{array}{l}\begin{array}{l}30.006310 \\
30.05180\end{array}\end{array}$ & $\begin{array}{l}179.069900 \\
179900950\end{array}$ & $\begin{array}{l}4563.0 \\
6453.5\end{array}$ & 25.6 & 25.6 & 339.8 \\
\hline & 8 & 140600 & 179.06970 & 4620.0 & & & \\
\hline 885 & $\begin{array}{l}0833.54 \\
0.54\end{array}$ & 30.01820 & $\begin{array}{r}179.07010 \\
179.07030\end{array}$ & $\begin{array}{l}4666.5 \\
4626.0\end{array}$ & $\begin{array}{r}-3.5 \\
-10.0\end{array}$ & $\begin{array}{l}35.5 \\
35.4\end{array}$ & $\begin{array}{l}356.8 \\
353.9\end{array}$ \\
\hline & $\begin{array}{l}8.54 \\
3.54\end{array}$ & $\begin{array}{l}0.99480 \\
.09640\end{array}$ & $\begin{array}{l}179.07030 \\
179.07020\end{array}$ & $\begin{array}{r}\begin{array}{r}5886.3 \\
4538.3\end{array} \\
4\end{array}$ & & & $\begin{array}{l}35 \\
35\end{array}$ \\
\hline & $\begin{array}{l}0848.54 \\
08835.54\end{array}$ & $\begin{array}{l}29.98300 \\
29.97130\end{array}$ & $\begin{array}{l}174.07010 \\
179 \\
1707000\end{array}$ & $\begin{array}{l}4449.0 \\
43860\end{array}$ & 23.8 & 48. & $\begin{array}{l}356.5 \\
352.5\end{array}$ \\
\hline & $\begin{array}{l}0855.54 \\
0903.54\end{array}$ & $\begin{array}{l}29.99560 \\
29.94780\end{array}$ & $\begin{array}{r}179.069900 \\
179 \\
1706820\end{array}$ & 42488 & & & \\
\hline & 0908.54 & 29.93000 & 179.00580 & 4141.5 & 18 & $\begin{array}{l}31.4 \\
59.8\end{array}$ & \\
\hline & $0 y$ & 1230 & 179.00090 & 3843.0 & & & 5.6 \\
\hline & $\begin{array}{l}0923.54 \\
0928.54 \\
0.54\end{array}$ & $\begin{array}{l}\begin{array}{l}29.90050 \\
29.88800\end{array}\end{array}$ & 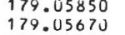 & $\begin{array}{l}\begin{array}{l}3575.3 \\
3314.3\end{array} \\
314\end{array}$ & $\begin{array}{l}500 \\
634\end{array}$ & $\begin{array}{l}101.8 \\
113.8\end{array}$ & $\begin{array}{r}348.0 \\
342.0\end{array}$ \\
\hline & 8.54 & $\begin{array}{l}29.87940 \\
29.86190\end{array}$ & $\begin{array}{l}179.055500 \\
179.05440\end{array}$ & $\begin{array}{l}3003.8 \\
2655.8\end{array}$ & & $\begin{array}{l}115.9 \\
136\end{array}$ & $\begin{array}{l}322.4 \\
319.7\end{array}$ \\
\hline & 0943.54 & 29.84970 & 179.05520 & 2235.0 & & & \\
\hline & $\begin{array}{l}0986.544 \\
0953.54\end{array}$ & $\begin{array}{l}29.8730 \\
29.82570\end{array}$ & $\begin{array}{l}179.05600 \\
179.05640\end{array}$ & $\begin{array}{l}1764.0 \\
1110.8\end{array}$ & $\begin{array}{l}800 \\
504\end{array}$ & $\begin{array}{l}177: 520 \\
202:\end{array}$ & $\begin{array}{l}299.0 \\
278.7\end{array}$ \\
\hline & $\begin{array}{l}5998.544 \\
1003.54\end{array}$ & 29.80230 & 179.05710 & 279.8 & & & 83.4 \\
\hline & 1008.54 & 79060 & & & & & 83.4 \\
\hline & 1018.54 & 29.70700 & 174.05850 & 707.22 & 76.0 & 237.8 & $\begin{array}{r}\begin{array}{r}292.9 \\
286.5\end{array} \\
286\end{array}$ \\
\hline & 8.54 & 75510 & $\begin{array}{l}179.05860 \\
179.05910\end{array}$ & $\begin{array}{r}975.0 \\
124.2\end{array}$ & & & \\
\hline & $\begin{array}{l}1033.55 \\
1303\end{array}$ & 29.73180 & 179.05990 & 1482.0 & 487 & 9. & $281^{\circ}$ \\
\hline & 1043.54 & 29.70870 & 179.00090 & 2092.5 & -832.5 & 00.8 & $\begin{array}{l}282.4 \\
294.9\end{array}$ \\
\hline & 1053.54 & 00540 & 179.00180 & 2412.0 & $=746.9$ & 7. & 293.9 \\
\hline & $\begin{array}{l}158.54 \\
103.54\end{array}$ & $\begin{array}{l}4.0 \\
80\end{array}$ & $\begin{array}{l}179.002240 \\
1799000990\end{array}$ & & -1056 & & \\
\hline & 113.54 & 29.05110 & 179.00330 & 3452.2 & -673 & 105.0 & 331.0 \\
\hline & 1195 & 30.0430 & 170.065 & 345 & & $105^{\circ}$ & $\begin{array}{l}344 . \\
343:\end{array}$ \\
\hline & $\begin{array}{l}1123.54 \\
1128.54\end{array}$ & $\begin{array}{l}29.691960 \\
29.61180\end{array}$ & 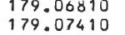 & $\begin{array}{l}\begin{array}{l}3129.0 \\
27710.3\end{array} \\
2\end{array}$ & $\begin{array}{l}-3550.5 \\
-334.6\end{array}$ & 80. & 271.5 \\
\hline & $\begin{array}{l}133.54 \\
1138.54\end{array}$ & $\begin{array}{l}6006060 \\
60150\end{array}$ & $\begin{array}{l}179.084000 \\
179.09390\end{array}$ & $\begin{array}{l}247 \\
234\end{array}$ & -334 & 1 & 265 \\
\hline & 1143.54 & 99700 & 179.16320 & 2359.5 & -33 & 105. & 203.0 \\
\hline & 1153.54 & $\begin{array}{l}\begin{array}{l}29.59250 \\
29.58840\end{array}\end{array}$ & 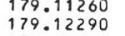 & $\begin{array}{l}\begin{array}{r}2524.5 \\
2965.5\end{array} \\
2965\end{array}$ & $\begin{array}{l}-331.0 \\
-330.1\end{array}$ & $\begin{array}{l}104.2 \\
103.0\end{array}$ & $\begin{array}{l}278.0 \\
307.2\end{array}$ \\
\hline & 1203.54 & .58 & $\begin{array}{l}179 \cdot 13 \\
179.14\end{array}$ & 3609.7 & & $89^{\circ}$ & 338.3 \\
\hline & 54 & 990 & & 38 & & & \\
\hline & 0.54 & 29.560000 & 179.17030 & 4167.0 & $\begin{array}{l}-300 \\
-300\end{array}$ & 64: & 351 \\
\hline & 8.54 & 55010 & 179.18710 & 23 & & & \\
\hline & $\begin{array}{l}1233.54 \\
1234.54\end{array}$ & 55110 & 17 & 4329.7 & & & 3539 \\
\hline & 1243.54 & 29.56 & 179.21220 & 4430.2 & -15 & 52.4 & 357.5 \\
\hline & $\begin{array}{l}.74 \\
.54\end{array}$ & $\begin{array}{l}\text { S } \\
: 5313020 \\
: 510\end{array}$ & $9 .<2060$ & $\begin{array}{l}46511.0 \\
451.2\end{array}$ & & & \\
\hline & 8.54 & $\begin{array}{l}5.2030 \\
52230\end{array}$ & .23870 & (5) & & & \\
\hline & & .51650 & 179.25830 & $\begin{array}{l}4562 \\
4620\end{array}$ & & & \\
\hline & & & 820 & 466 & & & \\
\hline & 54 & $\begin{array}{l}5.50120 \\
5.50120\end{array}$ & . & (7713.7 & -9 & ${ }^{43}$ & \\
\hline & & $\begin{array}{l}.49590 \\
.4950\end{array}$ & & $\begin{array}{l}485 \\
485\end{array}$ & & & \\
\hline & $\begin{array}{l}1338.554 \\
1336.54\end{array}$ & 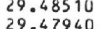 & $\begin{array}{l}9.31840 \\
9 \\
0.328000\end{array}$ & $\begin{array}{l}4893.7 \\
40920.7\end{array}$ & & & \\
\hline & & 29.47 & 179.34000 & $\begin{array}{l}4945.5 \\
40567\end{array}$ & 28 & & $381 . \mathrm{g}$ \\
\hline & & & 179.36380 & & & & \\
\hline & 8.54 & . & & 5094 & & & \\
\hline & 1418.54 & 29.44200 & 0.3 & & & & \\
\hline & & $\begin{array}{l}29.43770 \\
29.4340\end{array}$ & 10 & 520 & 8 & & 376.8 \\
\hline & $\begin{array}{l}1433.54 \\
1348\end{array}$ & 29.42910 & 170 & 5233.5 & $2060^{\circ}$ & 13. & $3740^{\circ}$ \\
\hline 285 & $\begin{array}{l}1448546 \\
1454\end{array}$ & 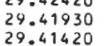 & 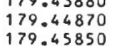 & 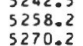 & 242.8 & 10.6 & 373.5 \\
\hline
\end{tabular}

TABLE 3 - Continued

\begin{tabular}{|c|c|c|c|c|c|c|c|}
\hline Day & Time & Latitude & Longitude & $\begin{array}{c}\text { DEPTH } \\
(\mathrm{m})\end{array}$ & $\begin{array}{c}\mathrm{MAG} \text { (âNOM } \\
(\mathrm{gammas})\end{array}$ & $\begin{array}{c}\text { FAA } \\
\text { (mgals) }\end{array}$ & $\begin{array}{c}\text { BGA } \\
\text { (mgals) }\end{array}$ \\
\hline 289 & 1453.54 & 29.60900 & 179.46840 & 5272.5 & 255.5 & 11.8 & 374.9 \\
\hline & $\begin{array}{l}14588.54 \\
1503.54\end{array}$ & $\begin{array}{r}29.403030 \\
29.39840\end{array}$ & $\begin{array}{l}179.478820 \\
179-48830\end{array}$ & 5262.0 & 274 & 18. & 376.6 \\
\hline 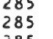 & $\begin{array}{l}1508.54 \\
1513.54 \\
153.5\end{array}$ & $\begin{array}{l}29.3929270 \\
29.3890 \\
28090\end{array}$ & $\begin{array}{l}179.999900 \\
79.50980 \\
79.5090\end{array}$ & $\begin{array}{l}5223.2 \\
5285.2 \\
582.2\end{array}$ & $\begin{array}{l}281.6 \\
285.2 .2 \\
285\end{array}$ & 8.6 & \\
\hline 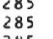 & & $\begin{array}{r}29.380090 \\
29.37480\end{array}$ & $\begin{array}{l}179.9525100 \\
779.53230\end{array}$ & $\begin{array}{l}5229.7 \\
5300.2 \\
500.2\end{array}$ & $\begin{array}{l}286.5 \\
281.7 \\
28.7\end{array}$ & $\begin{array}{l}6.3 \\
6.3\end{array}$ & 81.8 \\
\hline 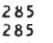 & $\begin{array}{l}15288.54 \\
1533.54\end{array}$ & $\begin{array}{l}29.368870 \\
29.36250\end{array}$ & $\begin{array}{l}179.554320 \\
779.553390\end{array}$ & $\begin{array}{l}5302.5 \\
5302.5\end{array}$ & $\begin{array}{l}\begin{array}{l}275.1 \\
264.4\end{array} \\
26.4\end{array}$ & $\begin{array}{r}10.1 \\
6.4\end{array}$ & $\begin{array}{l}375.2 \\
371.5\end{array}$ \\
\hline & $\begin{array}{l}1558.0 \\
15430\end{array}$ & $\begin{array}{l}29.356030 \\
29.34920\end{array}$ & $\begin{array}{l}1799.55450 \\
179.577480\end{array}$ & $\begin{array}{l}5229.3 \\
5295: 0\end{array}$ & $\begin{array}{l}\begin{array}{l}248.9 \\
229.2\end{array} \\
2.2\end{array}$ & 3.8 & 368.6 \\
\hline & $\begin{array}{l}1548.54 \\
1533.54\end{array}$ & $\begin{array}{l}29.342510 \\
29.35510\end{array}$ & $\begin{array}{l}179.5510 \\
179.59550\end{array}$ & $\begin{array}{l}5300.2 \\
5302.5\end{array}$ & $\begin{array}{l}204.8 \\
181.7\end{array}$ & 3.3 & $\begin{array}{l}308.3 \\
3380.9\end{array}$ \\
\hline & $\begin{array}{l}1556.54 \\
1000.54\end{array}$ & $\begin{array}{l}29.32920 \\
29.32350\end{array}$ & $\begin{array}{l}179.00590 \\
179.61650\end{array}$ & & $\begin{array}{l}159.5 \\
1387\end{array}$ & & \\
\hline & & $\begin{array}{l}29.31750 \\
29.31130\end{array}$ & & 5310.0 & 120.6 & $\because$ & 8.3 \\
\hline & 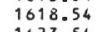 & 29.30510 & 179.04980 & 5295.0 & & & $\begin{array}{l}606.3 \\
60.5 \\
60.5\end{array}$ \\
\hline & $\begin{array}{l}10233.54 \\
1628.54\end{array}$ & $\begin{array}{l}29.29920 \\
29.29320\end{array}$ & $\begin{array}{l}179.0 .0100 \\
779.67230\end{array}$ & $\begin{array}{l}5284.5 \\
5274.8\end{array}$ & $\begin{array}{l}77.2 \\
68.8\end{array}$ & $\begin{array}{l}1.3 \\
3.3\end{array}$ & $\begin{array}{l}65.2 \\
66.5\end{array}$ \\
\hline & $\begin{array}{l}16333.54 \\
1038.54\end{array}$ & $\begin{array}{l}29.28800 \\
29.27820\end{array}$ & $\begin{array}{l}179.808240 \\
179.692210\end{array}$ & $\begin{array}{l}5272.5 \\
5722.5\end{array}$ & $\begin{array}{l}64.0 \\
59.5\end{array}$ & & \\
\hline & $\begin{array}{l}1643 . \\
1648 .\end{array}$ & $\begin{array}{l}29.27030 \\
3926303\end{array}$ & $\begin{array}{l}179.79170 \\
179.71180\end{array}$ & $\begin{array}{l}5272.5 \\
5262.0\end{array}$ & $\begin{array}{c}56.9 \\
55.8\end{array}$ & & \\
\hline & 1053. & $\begin{array}{l}29.25740 \\
39.25140\end{array}$ & $\begin{array}{l}179.77210 \\
170.73250\end{array}$ & 52.3 & $\begin{array}{l}55.7 \\
55.7\end{array}$ & 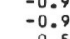 & $\begin{array}{l}36.1 \\
360 .\end{array}$ \\
\hline & 1703.54 & 29.26510 & 179.74300 & 5244.8 & $\begin{array}{l}55.7 \\
54.9\end{array}$ & & \\
\hline & & $\begin{array}{l}29.23890 \\
29.23270\end{array}$ & 80 & $\begin{array}{l}5235.5 \\
5235.0\end{array}$ & $\begin{array}{l}53.1 \\
51.0\end{array}$ & $\begin{array}{r}0.8 \\
-2.4\end{array}$ & \\
\hline & 1718. & $\begin{array}{l}29.22660 \\
28.9221960\end{array}$ & 179.773900 & $\begin{array}{l}52255.0 \\
539.2\end{array}$ & 48.9 & $0:$ & 60.6 \\
\hline & 1728.54 & 29.21090 & $\begin{array}{l}179.795270 \\
\end{array}$ & 5207.3 & $\begin{array}{l}47.1 \\
47.3\end{array}$ & $\begin{array}{l}-4.9 \\
-4.0\end{array}$ & 54.5 \\
\hline & $1738^{\circ}$ & 29.96000 & $\begin{array}{l}1798.8970 \\
179.81240\end{array}$ & $\begin{array}{l}5194.5 \\
5184.8\end{array}$ & $\begin{array}{l}48.8 \\
50.2\end{array}$ & 10.8 & \\
\hline & $\begin{array}{l}1773 . \\
1748 .\end{array}$ & $\begin{array}{l}29.19250 \\
29.18870\end{array}$ & $\begin{array}{l}19.882880 \\
179.83710\end{array}$ & $\begin{array}{l}51 \\
51\end{array}$ & $\begin{array}{l}52.6 \\
55.0\end{array}$ & & \\
\hline & $\begin{array}{l}1753.54 \\
1758.54\end{array}$ & $\begin{array}{l}29.18240 \\
29.17600\end{array}$ & $\begin{array}{l}199888810 \\
179885990\end{array}$ & 5144.3 & $\begin{array}{l}57.0 \\
58.1\end{array}$ & 5,0 & 359.2 \\
\hline & $\begin{array}{l}1803.545 \\
1808.54\end{array}$ & $\begin{array}{l}29.10780780 \\
29.15870\end{array}$ & $\begin{array}{l}179880780 \\
1798780\end{array}$ & $\begin{array}{l}5106.8 \\
5094.8\end{array}$ & 57 & -8. & 3.0 \\
\hline & & 14950 & 179.88310 & 5071.5 & & & $340^{\circ}$ \\
\hline & $\begin{array}{l}1888.54 \\
1823.54\end{array}$ & $\begin{array}{r}29.14250 \\
29.13700\end{array}$ & 179.90530 & $\begin{array}{l}50006.3 \\
5004.0\end{array}$ & $\begin{array}{l}49.1 \\
44.7\end{array}$ & $\begin{array}{l}14.2 \\
13.3\end{array}$ & \\
\hline & 1828. & $\begin{array}{l}29 \\
29\end{array}$ & $\begin{array}{r}19.91750 \\
179.92980\end{array}$ & $\begin{array}{l}4984.5 \\
4959.0\end{array}$ & & & 359. \\
\hline & 1838. & 30 & $\begin{array}{r}179.96210 \\
70.9546\end{array}$ & 39.5 & & & $35^{\circ}$ \\
\hline & 1848. & 29 & 179.96000 & $\begin{array}{r}88803 \\
488903\end{array}$ & $\begin{array}{l}52.4 \\
5.24\end{array}$ & 96 & 356.0 \\
\hline & 1858.54 & 29.10090 & 179.99070 & & & & \\
\hline & 1908. & 29.09050 & $\begin{array}{r}-179.98430 \\
-1\end{array}$ & $\begin{array}{l}4771.5 \\
4725: 8\end{array}$ & $\begin{array}{l}60.9 \\
63.6\end{array}$ & & $\begin{array}{l}3.3 \\
2.1\end{array}$ \\
\hline & $\begin{array}{l}1913.54 \\
1918.54\end{array}$ & $\begin{array}{l}29.08520 \\
29.07950\end{array}$ & $\begin{aligned}-179.997170 \\
-1779.95940\end{aligned}$ & $\begin{array}{l}4678.5 \\
4628.3\end{array}$ & $\begin{array}{l}66.8 \\
72.0\end{array}$ & 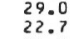 & $\begin{array}{l}355.2 \\
341.4\end{array}$ \\
\hline & & 000250 & & $\begin{array}{l}5 \\
8 \\
8\end{array}$ & & & \\
\hline & 1933.54 & 29.05400 & -179.92790 & 4489.5 & 93.7 & 28.8 & 37. \\
\hline 8 & $\begin{array}{l}1998.54 \\
1943.54\end{array}$ & $\begin{array}{l}29.04560 \\
29.03710\end{array}$ & $\begin{aligned} & 179.949170 \\
&-1799.90680\end{aligned}$ & $\begin{array}{l}458.8 \\
4426.5\end{array}$ & $\begin{array}{l}100.3 \\
106.9\end{array}$ & 32.2 & $\begin{array}{l}336.6 \\
337.0\end{array}$ \\
\hline & $\begin{array}{l}1948 \\
195 .\end{array}$ & $\begin{array}{l}29.033030 \\
29.02370\end{array}$ & $\begin{aligned} & 1798.895560 \\
&-1779886390\end{aligned}$ & $\begin{array}{r}4886.0 \\
4325.3\end{array}$ & $\begin{array}{l}112.2 \\
116.3\end{array}$ & $\begin{array}{l}37: 0 \\
41: 5 \\
41: 5\end{array}$ & $\begin{array}{l}339.0 \\
339.3\end{array}$ \\
\hline & 2003. & 29.01640 & $\begin{array}{r}-179.860090 \\
-1790\end{array}$ & 4225.5 & 3 & & 36. \\
\hline & $\begin{array}{l}2008 \\
2011\end{array}$ & $\begin{array}{l}28 \\
28\end{array}$ & $\begin{array}{r}1-19.888940 \\
-1799883780\end{array}$ & 60 & 4 & & \\
\hline & 2018 & 28.99250 & $\begin{array}{r}-179.82590 \\
\end{array}$ & 4035.8 & & 5 & 320 \\
\hline & $2030^{\circ}$ & 28.98230 & -179.8 & 3960.8 & 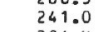 & & 34.5 \\
\hline 8 & $\begin{array}{l}2033 . \\
2038 .\end{array}$ & $\begin{array}{l}28.97720 \\
28.9720\end{array}$ & $\begin{aligned}-179.990000 \\
-1799.77800\end{aligned}$ & $\begin{array}{l}3882.5 \\
3822.8\end{array}$ & $\begin{array}{l}4.0 \\
4.2\end{array}$ & $\begin{array}{l}6.4 \\
9.7\end{array}$ & $\begin{array}{l}334: \\
332: 0\end{array}$ \\
\hline & 2045 & $\begin{array}{l}28.96710 \\
28.96190\end{array}$ & 9.7 & 3688.5 & & & \\
\hline & 2053 & 28.95590 & -179.74220 & 3033.0 & & & \\
\hline 8 & 203.54 & 28.94390 & $\begin{array}{l}-179.71820 \\
\end{array}$ & 3237.8 & $\begin{array}{l}520.4 \\
556.3\end{array}$ & $\begin{array}{l}91.1 \\
95.7\end{array}$ & 318.7 \\
\hline & & $\begin{array}{l}28.93190 \\
28\end{array}$ & & 234100 & & & \\
\hline & 3118. & 28.92590 & -179.082240 & 2242.5 & 5,3 & 130.9 & 35. \\
\hline 28 & 2128.54 & $\begin{array}{l}28.91990 \\
28.91390\end{array}$ & $\begin{array}{l}-179.65800 \\
-1\end{array}$ & 1701.0 & $\begin{aligned} 591.8 \\
512.0\end{aligned}$ & $\begin{array}{l}158.5 \\
158.7\end{array}$ & 275.8 \\
\hline & & & & & & & \\
\hline & $\begin{array}{l}2143.54 \\
2.54\end{array}$ & 28.89590 & -179.6 & .2 & 1.9 & & 79. \\
\hline 8 & 215 & 28.86390 & -179.59890 & $780=0$ & $\begin{array}{l}-850.4 \\
-835.0\end{array}$ & 81.0 & 11: \\
\hline & & & & & & & 0. \\
\hline & 2220 & $\begin{array}{l}28.850590 \\
\end{array}$ & $\begin{array}{l}-179.5 \\
-170.5\end{array}$ & & 197.8 & & 39. \\
\hline & 2218.54 & 28.85390 & -179.53930 & 1323.0 & 216.2 & 223.0 & $314^{\circ}$ \\
\hline & & & & & & & \\
\hline & & $\begin{array}{l}28.83 \\
28.83\end{array}$ & $77^{7 .}$ & & 11.8 & & \\
\hline & & & & & & & \\
\hline 28. & $\begin{array}{l}2248.54 \\
2253.54\end{array}$ & $\begin{array}{l}28.81880 \\
28.813\end{array}$ & 79.4 & $\begin{array}{l}3767.2 \\
4038.7\end{array}$ & $\begin{array}{c}85.1 \\
-0.9\end{array}$ & $\begin{array}{l}91.7 \\
78.3\end{array}$ & 56. \\
\hline & $\begin{array}{l}2258.54 \\
2303.54\end{array}$ & 10730 & 79. & & & & \\
\hline & & & & & & & \\
\hline & $\begin{array}{l}318.54 \\
318.54\end{array}$ & 年 & $\begin{array}{l}-418280 \\
40830\end{array}$ & & & $\begin{array}{l}41.2 \\
38.8\end{array}$ & 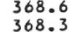 \\
\hline & & & 30 & & & & \\
\hline & & & & & 140 & & \\
\hline 8 & $\begin{array}{l}2338.54 \\
2343.54\end{array}$ & $\begin{array}{l}88.74560 \\
28.74000\end{array}$ & 然 & 485 & $\begin{array}{l}-285.6 \\
-283.0\end{array}$ & 41.3 & 36 \\
\hline 28 & $\begin{array}{l}2348.54 \\
2353.54\end{array}$ & $\begin{array}{l}28873350 \\
28.73070\end{array}$ & $\begin{array}{rl} & 1799.346020 \\
-1779 & 335500\end{array}$ & $\begin{array}{l}4618.5 \\
4598.2\end{array}$ & -440.4 & 43.6 & 360. \\
\hline 28 & $\begin{array}{l}300 \\
000\end{array}$ & 28.72 & -179. & 449 & & 6.3 & 56. \\
\hline & & $\begin{array}{l}20.71240 \\
28\end{array}$ & $\begin{aligned}-179.29020 \\
\end{aligned}$ & & $\begin{array}{l}-533.1 \\
-500.3 \\
-50.5\end{array}$ & & \\
\hline
\end{tabular}


TABLE 3 - Continued

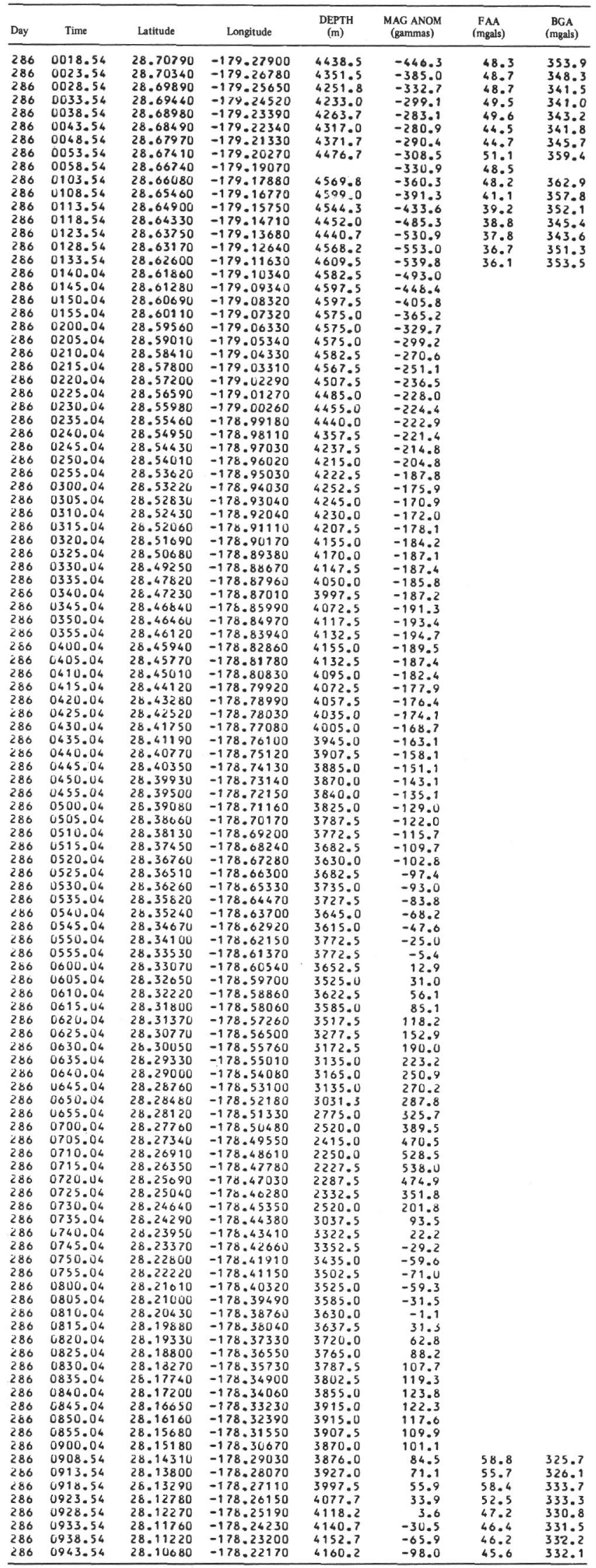

TABLE 3 - Continued

\begin{tabular}{|c|c|c|c|c|c|c|c|}
\hline Day & Time & Latitude & Longitude & $\begin{array}{c}\text { DEPTH } \\
(\mathrm{m})\end{array}$ & $\begin{array}{c}\text { MAG ANOM } \\
\text { (gammas) }\end{array}$ & $\begin{array}{c}\text { FAA } \\
\text { (mgals) }\end{array}$ & $\begin{array}{c}\text { BGA } \\
\text { (mgals) }\end{array}$ \\
\hline 286 & 0953.54 & 28.10150 & -1 & 4167.7 & -123.8 & 47.4 & .4 \\
\hline & $\begin{array}{l}9953.54 \\
0958.54\end{array}$ & $\begin{array}{l}28.096010 \\
28.09080\end{array}$ & $\begin{array}{l}-178.20150 \\
-178.19130\end{array}$ & & $\begin{array}{l}-145.5 \\
-166.1\end{array}$ & & \\
\hline 286 & 1003.54 & 28.08580 & -178.18090 & 4203.0 & -181.8 & & 339.8 \\
\hline & 1008.5 & 28.08080 & -178.17060 & & -200.3 & & 339.5 \\
\hline 286 & 1013.54 & 28.07000 & -178.16010 & 4220.2 & -216.9 & 52.9 & 343.5 \\
\hline $\begin{array}{l}2886 \\
286\end{array}$ & $\begin{array}{l}1018.54 \\
1023.54\end{array}$ & $\begin{array}{r}28.07130 \\
28.06660\end{array}$ & $\begin{array}{l}-178.16960 \\
-178\end{array}$ & 4222.5 & $\begin{array}{l}-220.1 \\
-2209.4\end{array}$ & & 346.8 \\
\hline 286 & 1028.54 & $\begin{array}{r}28.06660 \\
28.06180\end{array}$ & $\begin{array}{l}-178.139180 \\
-178.12850\end{array}$ & $\begin{array}{l}4080.8 \\
3757.5\end{array}$ & $\begin{array}{l}-209.4 \\
-180.7\end{array}$ & & $\begin{array}{l}341.4 \\
325.4\end{array}$ \\
\hline 286 & 1033.54 & 28.05700 & -178.11790 & 3 & & & 5.4 \\
\hline & & & -178.10720 & & & & $\begin{array}{l}319.2 \\
314.0\end{array}$ \\
\hline 286 & 1043.54 & 28.04710 & -178.09650 & 3129.0 & -9.0 & 97.7 & 313.2 \\
\hline 28 & 1048.54 & 28.04220 & -178.08600 & 2744.3 & 103.6 & 107.6 & 296.6 \\
\hline 286 & $\begin{array}{l}1053.54 \\
1058.54\end{array}$ & $\begin{array}{l}28.03730 \\
28.03240\end{array}$ & $\begin{array}{l}-178.07560 \\
-178.06510\end{array}$ & 2371.5 & $\begin{array}{l}247.6 \\
383.7\end{array}$ & 125.4 & 288.7 \\
\hline 286 & 1103.54 & $\begin{array}{r}28.03240 \\
28.02090\end{array}$ & -178.05380 & 1581.8 & $\begin{array}{l}385.7 \\
462.0\end{array}$ & $\begin{array}{l}164.7 \\
161.6\end{array}$ & 270.5 \\
\hline 286 & 1108.54 & & -178.04240 & & & & $\begin{array}{l}270.5 \\
264.3\end{array}$ \\
\hline & 1113.54 & 28.01580 & -178.03110 & 939.8 & 454. & $?$ & 4.5 \\
\hline 286 & 1118.54 & 28.01030 & -178.01970 & 751.5 & 349.3 & 213.6 & 265.3 \\
\hline 286 & 1123.56 & 28.00480 & -178.00860 & 594.0 & 143.3 & 231.9 & 272.8 \\
\hline 86 & 1128.54 & $\begin{array}{r}27.99960 \\
27.99480\end{array}$ & $\begin{array}{l}-177.99810 \\
-17798890\end{array}$ & 461.3 & -114.3 & 239.7 & 271.5 \\
\hline 286 & $\begin{array}{l}1133.54 \\
1138.54\end{array}$ & $\begin{array}{r}27.99480 \\
27.99000\end{array}$ & $\begin{array}{l}-177.98890 \\
-177\end{array}$ & $\begin{array}{l}338.3 \\
163.5\end{array}$ & $\begin{array}{l}-228.9 \\
-150.4\end{array}$ & 250.5 & 273.8 \\
\hline 286 & $\begin{array}{l}1138.54 \\
1143.54\end{array}$ & 27.98610 & -177.97210 & $\begin{array}{r}163.5 \\
89.3\end{array}$ & $\begin{array}{l}-110.4 \\
-102.2\end{array}$ & $\begin{array}{l}259.6 \\
279.7\end{array}$ & 0.9 \\
\hline & 1148.54 & 27.9820 & -17709630 & 32 & -286 & 4 & $\begin{array}{l}285.8 \\
296.4\end{array}$ \\
\hline 286 & 1153.54 & 27.97830 & -177.95680 & 67. & 366.1 & 296.7 & 301.3 \\
\hline 286 & 1158.54 & 27.97430 & -177.94940 & & 500.7 & 297.0 & \\
\hline 806 & $\begin{array}{l}1203.54 \\
120854\end{array}$ & $\begin{array}{l}27.97040 \\
27.96630\end{array}$ & $\begin{array}{l}-177.94200 \\
-177.93440\end{array}$ & 80.2 & 424.9 & 298.1 & 303.6 \\
\hline $\begin{array}{l}\begin{array}{l}2866 \\
286\end{array} \\
\end{array}$ & $\begin{array}{l}1208.54 \\
1213.54\end{array}$ & $\begin{array}{l}27.96630 \\
27.96230\end{array}$ & $\begin{array}{l}-177.93440 \\
-177.92680\end{array}$ & $\begin{array}{r}98.2 \\
168.0\end{array}$ & $\begin{array}{l}472.7 \\
320.0\end{array}$ & $\begin{array}{l}\begin{array}{l}296.9 \\
291.8\end{array}\end{array}$ & $\begin{array}{l}303.7 \\
303.4\end{array}$ \\
\hline & 1218 & 27. & 0 & & & & \\
\hline 286 & 1223.54 & $\begin{array}{r}27.95390 \\
27.94970\end{array}$ & $\begin{array}{l}-177.91300 \\
-177\end{array}$ & $\begin{array}{l}434.2 \\
514.5\end{array}$ & $\begin{array}{l}-276.6 \\
-4609\end{array}$ & 269.3 & 299.2 \\
\hline $\begin{array}{l}286 \\
286\end{array}$ & $\begin{array}{l}1228.54 \\
1233.54\end{array}$ & $\begin{array}{l}27.94970 \\
27.94570\end{array}$ & $\begin{array}{l}-177.90580 \\
-177\end{array}$ & 514.5 & $\begin{array}{l}-440.9 \\
-58550\end{array}$ & $\begin{array}{l}263.0 \\
258.3\end{array}$ & 298.4 \\
\hline & 1238.54 & 27.961 & -177 : & 632.2 & -711.6 & 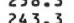 & $\begin{array}{l}297.5 \\
28008\end{array}$ \\
\hline 286 & 1243.54 & 27.93800 & -177.88380 & $\begin{array}{l}673.5 \\
67305\end{array}$ & -78 & 236.6 & $\begin{array}{r}280.0 \\
283.0\end{array}$ \\
\hline 286 & 1248.54 & 27.93430 & -177.87700 & 693.0 & -806.1 & 229.9 & 277.6 \\
\hline 286 & 1253.54 & 27.43050 & -177.87020 & 723.7 & -780.9 & 222.0 & 271.8 \\
\hline 286 & $\begin{array}{l}1258.54 \\
1303.54\end{array}$ & 27.92670 & $\begin{array}{l}-177.86340 \\
-177.85650\end{array}$ & 1008 & & 215.6 & 272,7 \\
\hline & & & -14 & & & & $r$ \\
\hline 286 & & & & & & & \\
\hline 286 & 1318.54 & 27.90 & -177 & 12 & -836.3 & 7 & 2 \\
\hline 286 & 1323.54 & 27.90 & -177.82790 & & -748.6 & 168.7 & 261.9 \\
\hline 38 & 34 & & -17 & & & & \\
\hline 286 & & & & & & & \\
\hline & & & & & & & \\
\hline 286 & & & & & & & \\
\hline 286 & & 27.87 & -17 & $2155^{\circ}$. & & 12 & 271.9 \\
\hline 286 & & & 0 & & & & \\
\hline 286 & $\begin{array}{l}1403.54 \\
1408.54\end{array}$ & $27-2$ & $-17-2-17$ & & & & \\
\hline 286 & 1413 & 27.85 & $\begin{array}{l}-177 \\
\end{array}$ & & 9 & & \\
\hline & & & & & & & \\
\hline $28.2-3$ & & & & & & & \\
\hline 286 & 1433.54 & .84020 & 77. 29 & 3294.1 & 79.0 & 40.8 & : 3 \\
\hline & & & & & & & \\
\hline
\end{tabular}

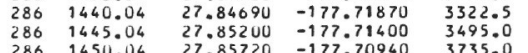

$\begin{array}{lllll}286 & 1450.04 & 27.85720 & -177.70960 & 3735.0 \\ 286 & 1455.04 & 27.86100 & -177.70400 & 3952.5\end{array}$

$\begin{array}{lllll}286 & 1455.04 & 27.86100 & -177.70400 & 3952.5 \\ 286 & 1500.04 & 27.8060 & -177.69860 & 3967.5 \\ 286 & 1505.04 & 27.87070 & -1777.69310 & 4967.5\end{array}$

$\begin{array}{lllll}286 & 1505.04 & 27.87070 & -177.69310 & 4117.5 \\ 286 & 1510.06 & 27.87020 & -177.68750 & 4267.5\end{array}$

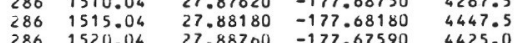

$\begin{array}{lllll}286 & 1520.04 & 27.88700 & -177.67590 & 4425.0 \\ 286 & 1525.04 & 27.89350 & -177.06980 & 447.5 \\ & 15350 & 27.99030 & -177.05600 & 4432.5\end{array}$

$\begin{array}{lllll}286 & 1530.04 & 27.89970 & -177.66330 & 447.5 \\ 286 & 1535.04 & 27.90630 & -177.05600 & 4432.5\end{array}$

$\begin{array}{lllll}286 & 1535.04 & 27.90630 & -177.05600 & 4432.5 \\ 286 & 1540.04 & 27.91300 & -177.06870 & 438.5 \\ 286 & 1545.04 & 27.91920 & -177.64250 & 4387.5\end{array}$

$\begin{array}{lllll}286 & 1545.04 & 27.91920 & -177.64250 & 4387.5 \\ 286 & 1550.04 & 27.92540 & -177.03200 & 425.0 \\ 280 & 1555.04 & 27.9350 & -177.6290 & 432.5\end{array}$

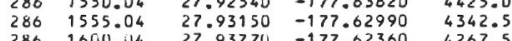

$\begin{array}{lllll}286 & 1600.04 & 27.93770 & -177.02360 & 4267.5 \\ 286 & 1605.04 & 27.94390 & -177.61730 & 4245.0\end{array}$

$\begin{array}{lllll}286 & 1010.04 & 27.955000 & -177.61080 & 422.5 \\ 286 & 1615.04 & 27.95750 & -177.60410 & 4207.5\end{array}$

$\begin{array}{lllll}286 & 1615.04 & 27.95750 & -177.60410 & 4207.5 \\ 286 & 1620.04 & 27.96440 & -177.59730 & 3885.0\end{array}$

$\begin{array}{lllll}286 & 1625.04 & 27.97150 & -177.59050 & 3630.0 \\ 286 & 1630.04 & 27.97850 & -177.5870 & 3517.5\end{array}$

$\begin{array}{lllll}286 & 1635.04 & 27.98550 & -177.57090 & 3322.5 \\ 286 & 1640.04 & 27.99210 & -177.57020 & 3187.5\end{array}$

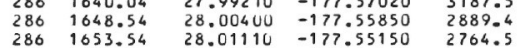

$\begin{array}{llll}286 & 1658.54 & 28.01190 & -177.54490 \\ 286 & 1703.54 & 28.01200 & -177.53830 \\ 286 & 1796.54 & 2800150 & -17.5328\end{array}$

$\begin{array}{llll}286 & 1703.54 & 28.01200 & -177.53830 \\ 286 & 1708.54 & 28.01550 & -177.53220\end{array}$

$\begin{array}{llll}286 & 1713.54 & 28.02250 & -177.52660 \\ 286 & 1718.54 & 28.02956 & -177.52100\end{array}$

$\begin{array}{llll}286 & 1723.54 & 28.03600 & -177.51550\end{array}$

$\begin{array}{llll}286 & 1728.54 & 28.04360 & -177.50990 \\ 286 & 1733.54 & 28.05060 & -177.50430 \\ 286 & 173654 & 28.05600 & -177.4510\end{array}$

$\begin{array}{llll}286 & 1733.54 & 28.03600 & -177.50430 \\ 286 & 1738.54 & 28.05040 & -177.4950 \\ 286 & 1743.54 & 28.00100 & -177.4830\end{array}$

$\begin{array}{llll}286 & 1743.54 & 28.06100 & -177.48230 \\ 280 & 1748.54 & 28.06570 & -177.47000\end{array}$

$\begin{array}{llll}286 & 1753.54 & 28.07170 & -177.46150 \\ 286 & 1758.54 & 28.07780 & -177.45300 \\ 286 & 17035 & 2800\end{array}$

$\begin{array}{llll}286 & 1803.54 & 28.08470 & -177.44570 \\ 286 & 1808.54 & 28.09200 & -137.43900\end{array}$

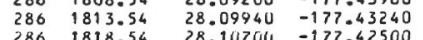

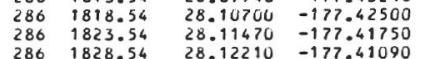

$\begin{array}{llll}286 & 1828.54 & 28.12210 & -177.41000 \\ 290 & 0255.04 & 28.10240 & -177.31720\end{array}$

$\begin{array}{llll}290 & 0255.04 & 28.10240 & -177.31720 \\ 290 & 0303.54 & 28.08290 & -177.30170\end{array}$

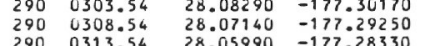

$\begin{array}{llll}290 & 0318.54 & 28.04840 & -1777.27420 \\ 290 & 032354 & 28.0340 & -177.26500\end{array}$

$\begin{array}{llll}290 & 0333.54 & 28.038900 & -177.26500 \\ 290 & 0328.54 & 28.02530 & -177.25580\end{array}$

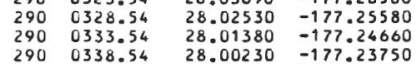

2613.8

2644.5
2678.2

2758.5

2849.5

2884.5
2874.0

2762.3
2774.2

2546.3

2370.8

1936.5
1587.0

1587.0

$\begin{array}{rr}79.9 & 160.1 \\ -58.4 & 110.3 \\ -57.1 & 95.2 \\ -77.5 & 85.7 \\ -54.0 & 77.4 \\ -34.6 & 69.0 \\ -33.8 & 60.1 \\ -32.1 & 51.3 \\ -28.4 & 44.8\end{array}$

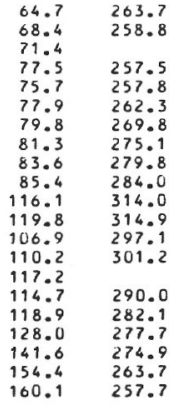

257.5 262.3 279.1 $314: 0$ 297.1

290.0 257.7 
TABLE 3 - Continued

TABLE 3 - Continued

\begin{tabular}{|c|}
\hline Day \\
\hline 290 \\
\hline 290 \\
\hline 290 \\
\hline $\begin{array}{l}290 \\
290\end{array}$ \\
\hline $\begin{array}{l}290 \\
290\end{array}$ \\
\hline 290 \\
\hline $\begin{array}{l}290 \\
290\end{array}$ \\
\hline 290 \\
\hline 290 \\
\hline $\begin{array}{l}290 \\
290\end{array}$ \\
\hline $\begin{array}{l}290 \\
290\end{array}$ \\
\hline 290 \\
\hline 290 \\
\hline 290 \\
\hline 290 \\
\hline $\begin{array}{r}290 \\
290\end{array}$ \\
\hline $\begin{array}{l}290 \\
290\end{array}$ \\
\hline 290 \\
\hline $\begin{array}{r}290 \\
290\end{array}$ \\
\hline 290 \\
\hline 290 \\
\hline $\begin{array}{r}290 \\
290\end{array}$ \\
\hline $\begin{array}{l}290 \\
290\end{array}$ \\
\hline 290 \\
\hline 290 \\
\hline 290 \\
\hline 290 \\
\hline $\begin{array}{r}290 \\
290\end{array}$ \\
\hline $\begin{array}{l}\begin{array}{l}290 \\
290\end{array}\end{array}$ \\
\hline 290 \\
\hline 290 \\
\hline 290 \\
\hline 290 \\
\hline $\begin{array}{l}290 \\
290\end{array}$ \\
\hline $\begin{array}{l}290 \\
290\end{array}$ \\
\hline 290 \\
\hline 290 \\
\hline 290 \\
\hline 290 \\
\hline 290 \\
\hline $\begin{array}{l}290 \\
290\end{array}$ \\
\hline 290 \\
\hline 290 \\
\hline 290 \\
\hline 290 \\
\hline 290 \\
\hline $\begin{array}{l}290 \\
290\end{array}$ \\
\hline $\begin{array}{l}290 \\
290\end{array}$ \\
\hline 290 \\
\hline 290 \\
\hline 290 \\
\hline $\begin{array}{l}290 \\
290\end{array}$ \\
\hline 290 \\
\hline 290 \\
\hline 290 \\
\hline 290 \\
\hline $\begin{array}{r}290 \\
290\end{array}$ \\
\hline 290 \\
\hline 290 \\
\hline 290 \\
\hline $\begin{array}{l}290 \\
290\end{array}$ \\
\hline $\begin{array}{l}290 \\
290\end{array}$ \\
\hline 290 \\
\hline 290 \\
\hline 290 \\
\hline 290 \\
\hline $\begin{array}{l}290 \\
290\end{array}$ \\
\hline \\
\hline 290 \\
\hline 290 \\
\hline 290 \\
\hline $\begin{array}{r}290 \\
290\end{array}$ \\
\hline 290 \\
\hline 290 \\
\hline 290 \\
\hline 290 \\
\hline 290 \\
\hline 290 \\
\hline $\begin{array}{l}290 \\
290\end{array}$ \\
\hline 290 \\
\hline 90 \\
\hline 290 \\
\hline 290 \\
\hline 290 \\
\hline 290 \\
\hline $\begin{aligned} 290 \\
290\end{aligned}$ \\
\hline 290 \\
\hline 290 \\
\hline 290 \\
\hline 290 \\
\hline 290 \\
\hline 290 \\
\hline 290 \\
\hline 290 \\
\hline $\begin{array}{r}290 \\
290\end{array}$ \\
\hline 290 \\
\hline 290 \\
\hline
\end{tabular}


TABLE 3 - Continued

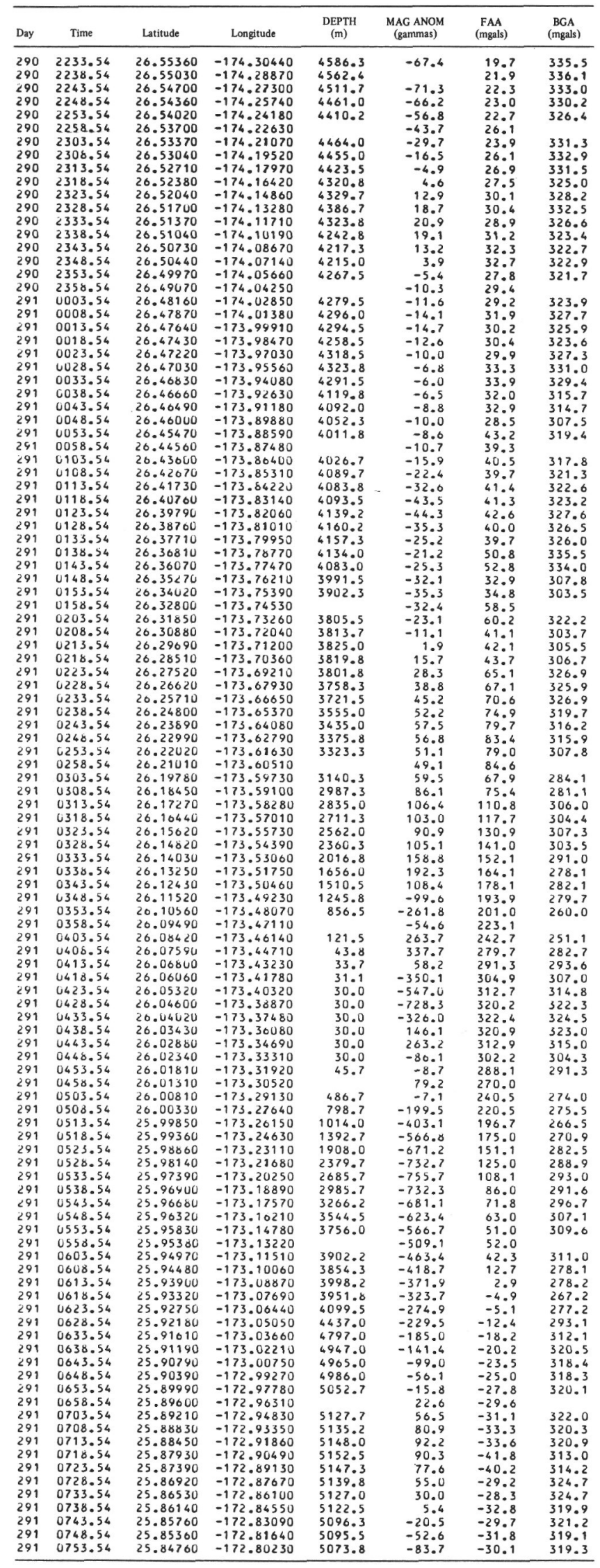

TABLE 3 - Continued

\begin{tabular}{|c|c|c|c|}
\hline Day & Time & Latitude & Longitude \\
\hline 291 & 0758.54 & 25.84150 & -172.78820 \\
\hline 291 & 0803.54 & 25.83680 & $\begin{array}{l}-172.77370 \\
-1\end{array}$ \\
\hline $\begin{array}{l}291 \\
291\end{array}$ & 0808.54 & $\begin{array}{l}25.83270 \\
25.82800\end{array}$ & $\begin{array}{l}-172.75900 \\
-172.74450\end{array}$ \\
\hline 291 & $\begin{array}{r}0818.54 \\
0854\end{array}$ & & $\begin{array}{l}-172.73020 \\
-172.7302\end{array}$ \\
\hline 201 & 0823.54 & 25.81990 & -172.71590 \\
\hline 291 & 0828.54 & 25.81520 & -172.70180 \\
\hline 291 & 0833.54 & 25.81060 & -172.68760 \\
\hline 291 & 0838.54 & 25.80590 & -172.67360 \\
\hline $\begin{array}{l}291 \\
291\end{array}$ & 0843.54 & 25.80100 & $\begin{array}{l}-172.65900 \\
-172\end{array}$ \\
\hline $\begin{array}{l}291 \\
291\end{array}$ & $\begin{array}{l}0848.54 \\
0853.54\end{array}$ & $\begin{array}{l}25.79580 \\
25.79070\end{array}$ & $\begin{array}{l}-172.64400 \\
-172.62990\end{array}$ \\
\hline 291 & 0858.54 & 25.78560 & -172.61530 \\
\hline 291 & 0903.54 & $\begin{array}{r}25.78070 \\
25\end{array}$ & -172.60080 \\
\hline & 0913.54 & 25.77100 & -172.57200 \\
\hline 291 & 0918.54 & 25.76610 & $-172.5576 \mathrm{U}$ \\
\hline 291 & 0923.54 & 25.76110 & -172.54360 \\
\hline 291 & 0928.54 & 25.75000 & -172.52920 \\
\hline $\begin{array}{l}291 \\
291\end{array}$ & 0933.54 & $\begin{array}{l}25.75090 \\
25.74570\end{array}$ & $\begin{array}{l}-172.51470 \\
-172\end{array}$ \\
\hline 291 & $\begin{array}{l}0938.54 \\
0.943 .54\end{array}$ & $\begin{array}{l}25.74570 \\
25.73990\end{array}$ & $\begin{array}{l}-172.50020 \\
-172.48640\end{array}$ \\
\hline 291 & & & \\
\hline 291 & 0953.54 & 25.72760 & -172.45950 \\
\hline 291 & 0958.54 & 25.72150 & -172.44600 \\
\hline 291 & 1003.54 & 25.71540 & -172.43260 \\
\hline $\begin{array}{l}299 \\
29\end{array}$ & 1008.54 & $\begin{array}{l}25.70930 \\
25.70310\end{array}$ & $\begin{array}{l}-172.41920 \\
-172.40570\end{array}$ \\
\hline 291 & 1018.54 & 25.69700 & -172.39230 \\
\hline 291 & 1023.54 & 25.69120 & -172.37850 \\
\hline 291 & 1028.54 & 25.68600 & -172.30420 \\
\hline 291 & 1033.54 & 25.08090 & -172.34980 \\
\hline 291 & 1038.54 & 25.67580 & -172.33540 \\
\hline $\begin{array}{l}291 \\
291\end{array}$ & 1063.54 & $\begin{array}{l}25.67070 \\
25.06564\end{array}$ & $\begin{array}{l}-172 \cdot 32090 \\
-172\end{array}$ \\
\hline 291 & $\begin{array}{l}1048.54 \\
1053.54\end{array}$ & $\begin{array}{l}25.60560 \\
25.60040\end{array}$ & $\begin{array}{l}-172.50630 \\
-172.29170\end{array}$ \\
\hline 291 & 1058.54 & 25.65500 & -172.27700 \\
\hline 291 & 1103.54 & 25.04980 & -172.26260 \\
\hline 291 & 1108.54 & 25.64480 & -172.24850 \\
\hline 291 & 1113.56 & 25.63980 & -172.23440 \\
\hline $\begin{array}{l}291 \\
291\end{array}$ & 1128.54 & $\begin{array}{l}25.03460 \\
25.02950\end{array}$ & $\begin{array}{l}-172.22020 \\
-172.20590\end{array}$ \\
\hline 291 & 1128.54 & 25.62420 & -172.19140 \\
\hline 291 & 1133.54 & 1830 & -172.17730 \\
\hline 291 & 1138.54 & 25.61040 & -172.10260 \\
\hline 291 & 1143.54 & 25.60260 & -172.14800 \\
\hline $\begin{array}{l}291 \\
291\end{array}$ & $\begin{array}{l}1148.54 \\
1153.54\end{array}$ & $\begin{array}{l}25.59470 \\
25558090\end{array}$ & $\begin{array}{l}-172.13330 \\
-172.11860\end{array}$ \\
\hline 291 & 1158.54 & 25.58300 & -172.10450 \\
\hline 291 & 1203.54 & 25.57970 & -172.09040 \\
\hline $\begin{array}{l}291 \\
291\end{array}$ & 1208.54 & $\begin{array}{l}25.57610 \\
2557240\end{array}$ & $\begin{array}{l}-172.07630 \\
-172.06210\end{array}$ \\
\hline 291 & $\begin{array}{l}1213.54 \\
1218.54\end{array}$ & $\begin{array}{l}25.57240 \\
25.56900\end{array}$ & $\begin{array}{l}-112.06210 \\
-172.04790\end{array}$ \\
\hline 291 & 1223.54 & 25.50550 & -172.03370 \\
\hline 29 & 1228.54 & 25.56200 & -172.01900 \\
\hline 291 & 1233.54 & 25.55840 & -172.00450 \\
\hline 299 & 1238.54 & 25.55490 & -171.99000 \\
\hline 299 & 1243.54 & $\begin{array}{l}25.55140 \\
2554780\end{array}$ & $\begin{array}{l}-171.97550 \\
-171.96100\end{array}$ \\
\hline $\begin{array}{l}291 \\
291\end{array}$ & $\begin{array}{l}12465.54 \\
1253.54\end{array}$ & $\begin{array}{l}25.54780 \\
25.54410\end{array}$ & $\begin{array}{l}-171.96100 \\
-171.9600\end{array}$ \\
\hline $\begin{array}{l}291 \\
291\end{array}$ & 1258.54 & $\begin{array}{l}25.54080 \\
25\end{array}$ & $\begin{array}{l}-171.93600 \\
-171\end{array}$ \\
\hline 291 & $\begin{array}{l}1303.54 \\
130854\end{array}$ & $\begin{array}{l}25.55030 \\
25.56370\end{array}$ & $\begin{array}{l}-171.92580 \\
-171.92270\end{array}$ \\
\hline 291 & 131.54 & 25.57830 & -171.92040 \\
\hline 291 & 1318.54 & 25.54220 & -171.91730 \\
\hline 291 & 1323.54 & 25.60000 & -171.91420 \\
\hline 291 & 1328.54 & 25.61960 & -171.91050 \\
\hline $\begin{array}{l}299 \\
291\end{array}$ & 1333.54 & $\begin{array}{l}25.63250 \\
255\end{array}$ & $\begin{array}{l}-171.90670 \\
-171\end{array}$ \\
\hline 291 & $\begin{array}{l}1338.54 \\
134354\end{array}$ & $\begin{array}{l}25.04530 \\
25.65850\end{array}$ & $\begin{array}{l}-171.90280 \\
-171.89850\end{array}$ \\
\hline 291 & 1348.54 & 25.67170 & -171.89420 \\
\hline 291 & 1353.54 & 25.08520 & -171.89000 \\
\hline 291 & 1358.54 & 25.69880 & -179.88580 \\
\hline 291 & 140 & 25.71220 & -171.88150 \\
\hline 291 & $\begin{array}{l}1408.54 \\
143.54\end{array}$ & $\begin{array}{l}25.72540 \\
25.73880\end{array}$ & $\begin{array}{l}-171.87730 \\
-171.87280\end{array}$ \\
\hline 291 & 1418.54 & 25.75210 & -171.86820 \\
\hline 29 & 1423.54 & 25.76300 & -171.86390 \\
\hline 291 & $\begin{array}{l}1428.54 \\
1433.54\end{array}$ & $\begin{array}{l}25.77520 \\
25.78270\end{array}$ & $\begin{array}{l}-171.85960 \\
-171.85660\end{array}$ \\
\hline 291 & 1438.54 & 25.78840 & -171. \\
\hline $\begin{array}{l}299 \\
291\end{array}$ & 1443.54 & & $\begin{array}{l}-171.85160 \\
-171\end{array}$ \\
\hline $\begin{array}{l}291 \\
291\end{array}$ & $\begin{array}{l}1448.54 \\
1453.54\end{array}$ & $\begin{array}{l}25.800560 \\
25.8050\end{array}$ & $\begin{array}{l}-171.84890 \\
-171.84510\end{array}$ \\
\hline $\begin{array}{l}29 \\
29\end{array}$ & 1458.54 & 25.81040 & -171.84080 \\
\hline 291 & $\begin{array}{l}1503.54 \\
150854\end{array}$ & 25.82130 & $\begin{array}{l}-171.83710 \\
-171.83304\end{array}$ \\
\hline & & $25>0$ & -1 \\
\hline 29 & is & 25.83 & -171 \\
\hline 29 & & 25.83 & $-1>>>>$ \\
\hline 29 & 15 & 0 & -17 \\
\hline 291 & & & \\
\hline 291 & $\begin{array}{l}1538.54 \\
1543.54\end{array}$ & 25.85850 & $\begin{array}{l}-171.80620 \\
-171.79940\end{array}$ \\
\hline & & & \\
\hline 291 & 15 & 86900 & -17 \\
\hline 291 & 15 & 0 & -1 \\
\hline 39. & & & \\
\hline & 1013.54 & & -1 \\
\hline $291>1>$ & 160 & & -1 \\
\hline & & & \\
\hline & 16 & & -1 \\
\hline 29 & 1633.54 & 25.91850 & -1 \\
\hline$<91$ & & & \\
\hline $291>-2$ & & & \\
\hline 291 & 4 & $25+2>$ & \\
\hline 0 & & & \\
\hline & 4 & 25.94 & $-171,71$ \\
\hline $291>$ & 1708.54 & & \\
\hline 201 & . & 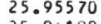 & no \\
\hline & $: 120.04$ & 25.90100 & -171.70000 \\
\hline
\end{tabular}


TABLE 3 - Continued

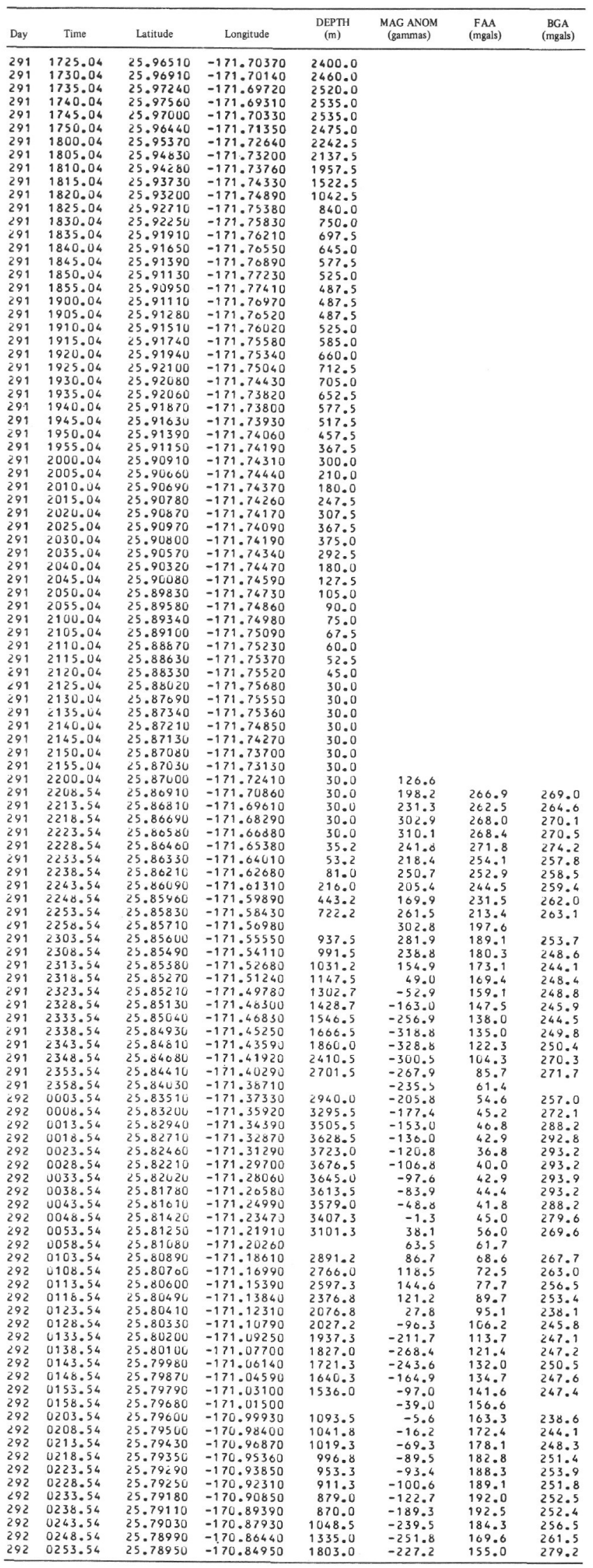

TABLE 3 - Continued

\begin{tabular}{|c|c|c|c|c|c|c|c|}
\hline Day & Time & Latitude & Longitude & 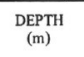 & $\begin{array}{l}\text { MAG ANOM } \\
\text { (gammas) }\end{array}$ & 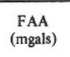 & 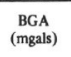 \\
\hline 292 & 0258.54 & 79040 & -170.83440 & & -191.1 & 146.1 & \\
\hline & $\begin{array}{l}03030.54 \\
0308.54\end{array}$ & $\begin{array}{l}255.79950 \\
25.79470\end{array}$ & $\begin{array}{r}-1770.81910 \\
-170.80380\end{array}$ & $\begin{array}{l}1949.3 \\
1972.5\end{array}$ & $\begin{array}{r}-177.55 \\
-193.8\end{array}$ & $\begin{array}{l}139.2 \\
137.4\end{array}$ & \\
\hline & $\begin{array}{l}30131.54 \\
0318.54\end{array}$ & $\begin{array}{l}25.79400 \\
25.79200\end{array}$ & $\begin{array}{r}-1700.78800 \\
-170.77280\end{array}$ & $\begin{array}{l}1995.0 \\
1953.0\end{array}$ & $\begin{array}{l}-230.4 \\
-267.4\end{array}$ & $\begin{array}{l}136.6 \\
137.9\end{array}$ & $\begin{array}{l}273.8 \\
272.4\end{array}$ \\
\hline 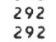 & $\begin{array}{l}0323.54 \\
0328.54\end{array}$ & $\begin{array}{l}25.789900 \\
255787440\end{array}$ & $\begin{array}{l}-170.75750 \\
-170.77260\end{array}$ & $\begin{array}{l}1887.8 \\
1904.2\end{array}$ & $\begin{array}{l}-289.1 \\
-297.3\end{array}$ & $\begin{array}{l}136.3 \\
138.8\end{array}$ & $\begin{array}{l}266.3 \\
269.9\end{array}$ \\
\hline$\frac{2}{2}$ & $\begin{array}{l}\begin{array}{r}33333.54 \\
0338.54\end{array}\end{array}$ & $\begin{array}{l}255.78500 \\
25.78250\end{array}$ & $\begin{array}{r}-1700.72770 \\
-170.71200\end{array}$ & $\begin{array}{l}1909.5 \\
1905.0\end{array}$ & $\begin{array}{l}-300.2 \\
-3378.8\end{array}$ & $\begin{array}{l}139.5 \\
149: 1\end{array}$ & 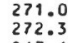 \\
\hline $\begin{array}{ll}2922 \\
292\end{array}$ & $\begin{array}{l}30343.54 \\
0348.54\end{array}$ & $\begin{array}{l}25.788010 \\
25.77790\end{array}$ & $\begin{array}{l}-1770.609000 \\
-170.68270\end{array}$ & $\begin{array}{l}1810.5 \\
1759.5 \\
175\end{array}$ & 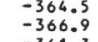 & $\begin{array}{l}1126.9 \\
149.0\end{array}$ & $\begin{array}{l}276.6 \\
270.2\end{array}$ \\
\hline & $\begin{array}{r}35535.54 \\
0358.54\end{array}$ & $\begin{array}{l}.77590 \\
777390\end{array}$ & $\begin{array}{r}1700.67000 \\
-170.55130\end{array}$ & & $\begin{array}{l}-361.3 \\
-349.7\end{array}$ & $\begin{array}{r}149.7 \\
149.0\end{array}$ & \\
\hline & $\begin{array}{l}0403.54 \\
0408.54\end{array}$ & $\begin{array}{l}25.77200 \\
25.77700\end{array}$ & $\begin{aligned}-1770.63560 \\
-170.01990\end{aligned}$ & $\begin{array}{l}1951.5 \\
2003.2\end{array}$ & $\begin{array}{l}-337.5 \\
-336.7\end{array}$ & $\begin{array}{l}145.2 \\
139.9\end{array}$ & 9.6 \\
\hline $\begin{array}{l}292 \\
292\end{array}$ & $\begin{array}{l}40133.54 \\
0418.54\end{array}$ & $\begin{array}{l}25.70800 \\
25.76590\end{array}$ & $\begin{array}{r}-1700.042020 \\
-170.58870\end{array}$ & $\begin{array}{l}2067.7 \\
2076.8\end{array}$ & $\begin{array}{l}-3.48 .8 \\
-351.8 \\
-3\end{array}$ & $\begin{array}{l}137.0 \\
129.6\end{array}$ & 272.6 \\
\hline & $\begin{array}{l}0628854 \\
04284\end{array}$ & 25.76000 & $\begin{array}{l}-100.55820 \\
\end{array}$ & 2309.2 & $\begin{array}{l}-317 \\
-317\end{array}$ & & 87.0 \\
\hline & & 75980 & -170.56150 & 46.5 & -295.9 & & \\
\hline & 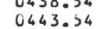 & 78620 & 17050850 & $\begin{array}{l}2433.8 \\
2538.0\end{array}$ & 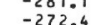 & & (3) \\
\hline & 8.54 & 75390 & -170.49290 & 2598.0 & $-26 \quad 0$ & 103.3 & \\
\hline & 然 & 7. & 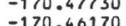 & & & & \\
\hline & 03.54 & & -170.46610 & 2801.2 & -230 & 87 & 80.6 \\
\hline $\begin{array}{l}29 \\
29\end{array}$ & $\begin{array}{l}5508.54 \\
0513.54\end{array}$ & $\begin{array}{l}25.7440 \\
25.74140\end{array}$ & $\begin{array}{r}-1700.43050 \\
-170.44480\end{array}$ & 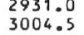 & $\begin{array}{l}-2232.3 \\
-232.8 \\
-25\end{array}$ & $\begin{array}{l}84.6 \\
79.9\end{array}$ & $\begin{array}{l}288.2 \\
280.0\end{array}$ \\
\hline & $\begin{array}{l}\begin{array}{l}5518.54 \\
0523.54\end{array} \\
0.54\end{array}$ & $\begin{array}{l}25.73870 \\
25.73600\end{array}$ & $\begin{array}{l}-1700.39910 \\
-170.36300\end{array}$ & $\begin{array}{l}3085.5 \\
31335.5\end{array}$ & $\begin{array}{l}-219 \\
-2191\end{array}$ & 66 & $\begin{array}{l}283 . \\
282:\end{array}$ \\
\hline & 0528.54 & .73350 & -170.36740 & 3237.0 & & & \\
\hline 24. & $\begin{array}{l}55338.54 \\
0538.54\end{array}$ & $\begin{array}{l}25.7300 \\
25.72820\end{array}$ & $\begin{array}{l}-1700.35270 \\
-170.33720\end{array}$ & $\begin{array}{l}\begin{array}{r}3282.7 \\
3332.2\end{array} \\
332\end{array}$ & $\begin{array}{l}-133.7 \\
-132.6\end{array}$ & 49.7 & $\begin{array}{l}275.8 \\
280.9\end{array}$ \\
\hline & 0548.54 & 25.72360 & $\begin{array}{l}-17003750 \\
-170.30570\end{array}$ & 3433.5 & & & \\
\hline & & & -170.29070 & 3458.2 & -188 & 33.5 & 7ii: \\
\hline 29 & 0603.54 & $\begin{array}{l}3.7170 \\
25.71480\end{array}$ & $\begin{array}{l}-170.26000 \\
-170.26160\end{array}$ & 3475.5 & $\begin{array}{l}-210.6 \\
-210.3\end{array}$ & 22 & 201.4 \\
\hline & & 0 & 60 & 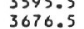 & $\begin{array}{l}-2236 \\
-236\end{array}$ & & \\
\hline & 8.54 & 25.70660 & $\begin{array}{l}-1700.211470 \\
-120\end{array}$ & 3700.5 & $-23 z$ & 37.2 & 292.0 \\
\hline 29 & 0028.54 & 25.70240 & -170.17820 & 3771.00 & $\begin{array}{l}-218.9 \\
\end{array}$ & & $\begin{array}{r}256.3 \\
256.3\end{array}$ \\
\hline & 0658.54 & 25.69830 & -170.15270 & $\begin{array}{l}3828.0 \\
382.0\end{array}$ & -1 & & 253: \\
\hline & 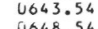 & $\begin{array}{l}25.696909 \\
2096\end{array}$ & -170.13650 & $\begin{array}{l}3853.5 \\
389.5\end{array}$ & -185 & 10. & 275.5 \\
\hline 29 & 0053.54 & 25.69400 & -170.10120 & $\begin{array}{l}3918.7 \\
3.9\end{array}$ & $\begin{array}{l}-188.4 \\
-200.4\end{array}$ & $4:$ & $74: 6$ \\
\hline & 074 & 25.69050 & -170.00980 & 3996.0 & -234 & & 5. \\
\hline & $\begin{array}{l}0708.54 \\
0713.54\end{array}$ & $\begin{array}{l}25.68800 \\
25.68600\end{array}$ & $\begin{array}{r}-1700.05440 \\
-170.03910\end{array}$ & $\begin{array}{l}0020.7 \\
4064.2\end{array}$ & $\begin{array}{l}-255.8 \\
-277.4\end{array}$ & $\begin{array}{r}-13.9 \\
-15.4\end{array}$ & 264.5 \\
\hline 29 & $\begin{array}{l}0718.54 \\
0723.54\end{array}$ & $\begin{array}{l}25.68490 \\
25.68330\end{array}$ & $\begin{array}{r}-1720.02390 \\
-170.00890\end{array}$ & $\begin{array}{r}0090.5 \\
4142.2\end{array}$ & $\begin{array}{l}-299.4 \\
-313.6\end{array}$ & $\begin{array}{l}-16.9 \\
-19.7\end{array}$ & $\begin{array}{l}264.8 \\
265.5\end{array}$ \\
\hline $\begin{array}{l}292 \\
292\end{array}$ & $\begin{array}{r}0728.54 \\
0733.54\end{array}$ & $\begin{array}{l}5.68170 \\
25.68010\end{array}$ & $\begin{array}{l}-1609.99580 \\
-169.97880\end{array}$ & $\begin{array}{l}4141.5 \\
4127.3\end{array}$ & $\begin{array}{l}-321.5 \\
-334.5\end{array}$ & $\begin{array}{l}-19.0 \\
-19.6\end{array}$ & 264.6 \\
\hline & or & 25.67000 & -169.94840 & 4083.8 & -33 & -18.8 & $\begin{array}{l}262.4 \\
262.4\end{array}$ \\
\hline 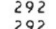 & $\begin{array}{l}074.54 .54 \\
0735.54\end{array}$ & $\begin{array}{l}25.67520 \\
25.6720\end{array}$ & $\begin{array}{r}-1699.93290 \\
-1\end{array}$ & $\begin{array}{l}40888.22 \\
4080.8\end{array}$ & $\begin{array}{l}-323.8 \\
-3218.8\end{array}$ & $\begin{array}{l}-16.6 \\
-15.6\end{array}$ & 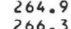 \\
\hline & $\begin{array}{l}58.54 \\
0.534\end{array}$ & 80 & $-1 \epsilon$ & 4069,3 & $\begin{array}{l}-29.7 \\
-285\end{array}$ & $\begin{array}{r}-15.6 \\
-13.8\end{array}$ & 650 \\
\hline & 0808.54 & 25.06960 & -169.87100 & 4021.5 & -276 & -10.7 & 63.0 \\
\hline $\begin{array}{l}296 \\
292\end{array}$ & $\begin{array}{l}0813.54 \\
0818.54\end{array}$ & $\begin{array}{l}25.06800 \\
25.06007\end{array}$ & $\begin{array}{r}-1699.8550 \\
-169.84010\end{array}$ & $\begin{array}{l}\begin{array}{r}3981.0 \\
3930.8\end{array} \\
3.8\end{array}$ & $\begin{array}{l}-2565.6 \\
-250.8\end{array}$ & $\begin{array}{l}-8.1 \\
-5.0\end{array}$ & 26 \\
\hline 292 & $\begin{array}{l}80233.54 \\
0828.54\end{array}$ & $\begin{array}{l}25.665510 \\
25.66330\end{array}$ & $\begin{array}{r}-169988240 \\
-169.80870\end{array}$ & $\begin{array}{l}\begin{array}{r}3894.0 \\
3832.5\end{array} \\
385\end{array}$ & -228.4 & 4.3 & 268.2 \\
\hline & $\begin{array}{l}0838.54 \\
0838.54\end{array}$ & $\begin{array}{l}25.66190 \\
25.6500\end{array}$ & 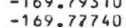 & 3615.0 & $\begin{array}{l}-202.0 \\
-202 .\end{array}$ & & 202.2 \\
\hline 29 & 0843.54 & 25.05816 & -169.7017170 & 3540.0 & -188 & & \\
\hline 29 & 0853.54 & 25.65490 & -169.73070 & 3402.8 & $\begin{array}{l}-152: 1 \\
-1520\end{array}$ & $27: 0$ & 261.3 \\
\hline & & 0 & $\begin{array}{l}-1094.6 \\
-109.6\end{array}$ & 3187.5 & 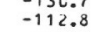 & & 599.1 \\
\hline 29 & 0908.54 & 25.04980 & -169.68350 & $\begin{array}{l}3133.5 \\
313.5\end{array}$ & -90 & $455-2$ & 260.9 \\
\hline 292 & 0918.54 & 25.64680 & -169.65280 & 2984.3 & -44.4 & 58.0 & 263.5 \\
\hline & 0928.54 & 25.64320 & -169.62130 & & & & \\
\hline $\begin{array}{l}922 \\
292\end{array}$ & $\begin{array}{l}9933.54 \\
0938.54\end{array}$ & $\begin{array}{l}25.64130 \\
25.03940\end{array}$ & 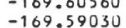 & $\begin{array}{l}\begin{array}{l}2297.3 \\
1854.0\end{array} \\
18\end{array}$ & $\begin{array}{l}16 \\
52\end{array}$ & 11 & 48. \\
\hline $\begin{array}{lll}292 \\
292\end{array}$ & $\begin{array}{l}694,04 \\
0948\end{array}$ & $\begin{array}{l}25 . \\
25 .\end{array}$ & $\begin{array}{l}-169.5 \\
-1090^{5}\end{array}$ & $\begin{array}{r}1186.5 \\
611.3\end{array}$ & $\begin{array}{l}100 \\
180\end{array}$ & & 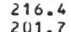 \\
\hline & & $\begin{array}{l}25.03 \\
25.63\end{array}$ & -1 & 375.8 & 241 & & \\
\hline & 54 & 25.63020 & -169. & ts:.8 & 278 & & \\
\hline 29 & 1013.54 & 25.02040 & -169 & 41 & 220.1 & & \\
\hline & 14 & 25 & $\begin{array}{l}-169 . \\
-169 .\end{array}$ & & $\begin{array}{l}324 \\
312\end{array}$ & & \\
\hline 24 & $\begin{array}{l}1028.54 \\
1033.54\end{array}$ & $\begin{array}{l}25.620400 \\
25.61930\end{array}$ & $\begin{array}{l}-109.45720 \\
-1090.44580\end{array}$ & $\begin{array}{l}30.0 \\
30.0\end{array}$ & $\begin{array}{l}\begin{array}{l}414.3 \\
498.3\end{array} \\
4987\end{array}$ & $\begin{array}{l}263 \\
263\end{array}$ & \\
\hline $\begin{array}{ll}29 \\
29\end{array}$ & $\begin{array}{l}1038.545 \\
103545\end{array}$ & 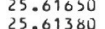 & $\begin{array}{c}-169 \\
-169 \\
-109\end{array}$ & 30 & $\begin{array}{l}429 \\
323\end{array}$ & & \\
\hline & 54 & 25.00970 & & 30.0 & 157 & & \\
\hline & & 25.66 & & & -80 & & \\
\hline 29 & & $\begin{array}{l}25.59600 \\
25.59200\end{array}$ & $\begin{array}{l}-169 . \\
-169 .\end{array}$ & 8.5 & $\begin{array}{l}-44.6 \\
285.3 \\
28.3\end{array}$ & $\begin{array}{l}27 \\
25\end{array}$ & \\
\hline & & & & & & & 69 \\
\hline & & 0 & -169. & 123 & 345 & & \\
\hline & & & 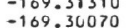 & $\begin{array}{l}15085.2 \\
1565\end{array}$ & 2313.0 & 9.6 & \\
\hline & & & & 175 & 13.1 & & \\
\hline & & & 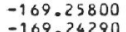 & 2630.2 & 85 & & \\
\hline & & 25.54320 & -169.222780 & & 127 & 33. & \\
\hline & 1208.54 & 25.53330 & & 81 & 95.7 & 8 & \\
\hline & $\begin{array}{l}3.54 \\
8.54\end{array}$ & $\begin{array}{l}5.52860 \\
5.52330\end{array}$ & $\begin{array}{l}-1009.18240 \\
-1090 \\
-16720\end{array}$ & $\begin{array}{l}\begin{array}{l}38288.0 \\
3837.7\end{array} \\
3\end{array}$ & $\begin{array}{l}889.6 \\
83.4\end{array}$ & $-2: 0$ & $\begin{array}{l}260.6 \\
262.3\end{array}$ \\
\hline
\end{tabular}


TABLE 3 - Continued

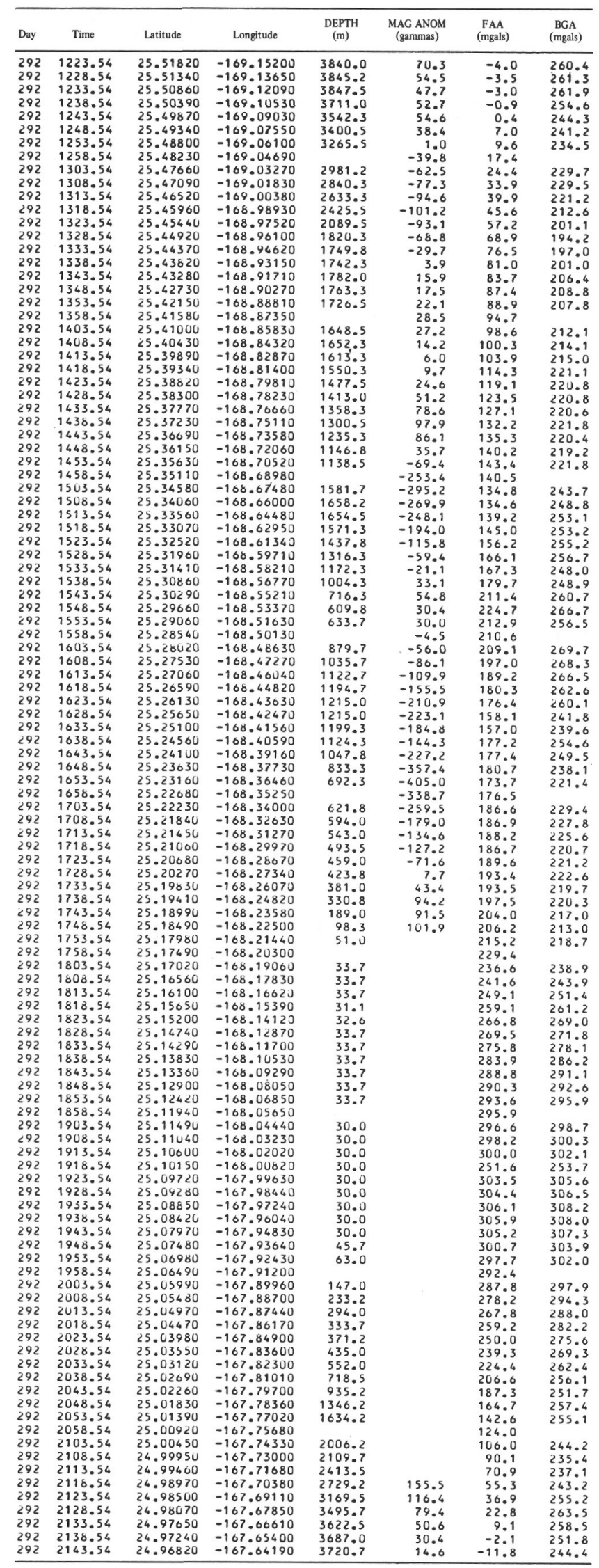

TABLE 3 - Continued

\begin{tabular}{|c|c|c|c|c|c|c|c|}
\hline ay & Time & Latitude & Longitude & $\begin{array}{c}\text { DEPTH } \\
(\mathrm{m})\end{array}$ & $\begin{array}{c}\text { MAC ANOM } \\
\text { (Rammas) }\end{array}$ & 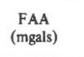 & $\begin{array}{l}\text { SGA } \\
\text { (mgats) }\end{array}$ \\
\hline 292 & $\begin{array}{l}2148.54 \\
2153.54\end{array}$ & $\begin{array}{l}24.96410 \\
24.959980\end{array}$ & $\begin{array}{l}-167.63000 \\
-167.61810\end{array}$ & $\begin{array}{l}3732.7 \\
37602\end{array}$ & 1.9 & $\begin{array}{l}-18.0 \\
-32.0\end{array}$ & $\begin{array}{l}239.0 \\
234.0\end{array}$ \\
\hline $\begin{array}{ll}92 \\
92 \\
92\end{array}$ & $\begin{array}{l}21533.5 \\
2158.5\end{array}$ & $\begin{array}{l}24.959990 \\
24.95570\end{array}$ & $\begin{array}{l}-167 \\
-167 . \\
-10\end{array}$ & & & & \\
\hline 92 & $\begin{array}{l}22203.54 \\
220854\end{array}$ & $\begin{array}{l}24.99110 \\
24.94630\end{array}$ & $\begin{array}{l}-167.594440 \\
-167.58260\end{array}$ & $\begin{array}{l}3742.5 \\
3742.5\end{array}$ & $\begin{array}{l}-18.0 \\
-21.1\end{array}$ & $\begin{array}{l}-288.6 \\
-31.8\end{array}$ & \\
\hline 92 & $\begin{array}{l}2213.54 \\
2218.54\end{array}$ & $\begin{array}{l}22.94150150 \\
24: 93690\end{array}$ & $\begin{array}{l}-167.575080 \\
-167.559900\end{array}$ & $\begin{array}{l}\begin{array}{l}37377.3 \\
3735.0\end{array} \\
375\end{array}$ & $\begin{array}{l}-22.4 \\
-22.5\end{array}$ & $\begin{array}{l}-34.7 \\
-36.6\end{array}$ & 20.6 \\
\hline & 228. & 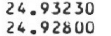 & $\begin{array}{l}-167.56730 \\
-167.53500\end{array}$ & $\begin{array}{l}3729.8 \\
3727.5\end{array}$ & & $\begin{array}{l}-3778 \\
-34.3\end{array}$ & \\
\hline & 233. & & -167.52330 & & & & \\
\hline 92 & $\begin{array}{l}2238.54 \\
2243.54\end{array}$ & 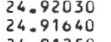 & $\begin{array}{l}-167.511880 \\
-167.50010\end{array}$ & $\begin{array}{l}3727.5 \\
3727.5\end{array}$ & $\begin{array}{l}-23.0 \\
-19.4 \\
-19.4\end{array}$ & $\begin{array}{l}-37.1 \\
-37: 0\end{array}$ & 9.6 \\
\hline 92 & $\begin{array}{l}2228.5354 \\
2253.54\end{array}$ & $\begin{array}{l}24.91250 \\
24.908500\end{array}$ & $\begin{array}{l}-167.688860 \\
-167.476700\end{array}$ & $\begin{array}{l}\begin{array}{l}37222.3 \\
3720.0\end{array} \\
3\end{array}$ & $\begin{array}{l}-15.3 \\
-11.2 \\
-11.2\end{array}$ & $\begin{array}{l}-35.5 \\
-33.8\end{array}$ & \\
\hline & $\begin{array}{l}2258.5 \\
2303 .\end{array}$ & $\begin{array}{l}99470 \\
90090\end{array}$ & $\begin{array}{l}65900 \\
5300\end{array}$ & 3707.3 & $\begin{array}{l}-7.2 \\
-2.5\end{array}$ & & \\
\hline 92 & $\begin{array}{l}2308.54 \\
2331.54\end{array}$ & $\begin{array}{l}24.89720 \\
24.89360\end{array}$ & $\begin{array}{l}-167.44100 \\
-167.42890\end{array}$ & $\begin{array}{l}3689.3 \\
3606.8\end{array}$ & 1.1 & $\begin{array}{l}-21.0 \\
-14.7\end{array}$ & \\
\hline 92 & $\begin{array}{l}2318.54 \\
2323.54\end{array}$ & $\begin{array}{l}24.88990 \\
24.886010\end{array}$ & $\begin{array}{r}-167.47710 \\
-167.40540\end{array}$ & $\begin{array}{l}3633.8 \\
3501: 8\end{array}$ & $\begin{array}{l}16.4 \\
29.0\end{array}$ & $\begin{array}{l}-9.5 \\
0.8\end{array}$ & \\
\hline $\begin{array}{l}292 \\
292 \\
292\end{array}$ & $\begin{array}{l}2328.54 \\
23335.54 \\
2334\end{array}$ & $\begin{array}{l}24.88210 \\
24.87790\end{array}$ & $\begin{array}{l}-167.39360 \\
-167.38170\end{array}$ & $\begin{array}{l}3324.0 \\
3128.3\end{array}$ & $\begin{array}{l}50.8 \\
76.6\end{array}$ & $\begin{array}{l}12.5 \\
25.0\end{array}$ & .4 \\
\hline tol & $\begin{array}{l}2338.54 \\
2343.54 \\
2.54\end{array}$ & $\begin{array}{l}24.87390 \\
24.87000\end{array}$ & $\begin{array}{r}-167.373000 \\
-167.35850\end{array}$ & $\begin{array}{l}2810.3 \\
2306.3\end{array}$ & $\begin{array}{l}115.4 \\
156.0 \\
150.0\end{array}$ & $\begin{array}{l}37.1 \\
53: 8\end{array}$ & $\begin{array}{l}230.6 \\
212.6 \\
212.6\end{array}$ \\
\hline & & $\begin{array}{l}8.86170 \\
86170\end{array}$ & 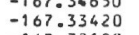 & 1269.8 & 198.1 & 102.4 & 189.8 \\
\hline & 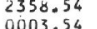 & $\begin{array}{l}24.885750 \\
24.85340\end{array}$ & $\begin{array}{l}=167.32190 \\
=167.31030\end{array}$ & 1027.5 & 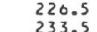 & $\begin{array}{l}125.3 \\
115.5\end{array}$ & \\
\hline 293 & 0008.54 & 24.84920 & -167.29870 & 936.8 & 215.9 & 100.8 & \\
\hline & $0018:$ & & & & & 187.2 & \\
\hline 93 & 8.54 & $\begin{array}{l}24.886090 \\
2483260\end{array}$ & $\begin{array}{l}7 \\
7.202020 \\
7\end{array}$ & $\begin{array}{l}843.8 \\
795.8\end{array}$ & & $\begin{array}{l}200.0 \\
211.6\end{array}$ & \\
\hline & & & 87.24120 & 774.8 & & 222.4 & \\
\hline 93 & $\begin{array}{l}\begin{array}{l}6038.54 \\
00636.54\end{array} \\
0.54\end{array}$ & $\begin{array}{l}24.82400 \\
24.81970\end{array}$ & $\begin{array}{l}-167.22970 \\
-167.21820\end{array}$ & $\begin{array}{l}798.7 \\
836.2^{2}\end{array}$ & $\begin{array}{l}\begin{array}{l}351.7 \\
253.4\end{array} \\
253\end{array}$ & $\begin{array}{l}22889 \\
229.8\end{array}$ & 年 \\
\hline $\begin{array}{l}293 \\
293\end{array}$ & $\begin{array}{l}\begin{array}{l}0048.54 \\
0053.54\end{array} \\
0.5\end{array}$ & $\begin{array}{l}24.81540 \\
24.81120\end{array}$ & $\begin{array}{l}-167.20690 \\
-167.19550\end{array}$ & $\begin{array}{l}842.3 \\
850.5\end{array}$ & $\begin{aligned} & 44.9 \\
-21: 7 & \end{aligned}$ & $\begin{array}{l}2224.1 \\
218.2\end{array}$ & $6.8 \quad-8$ \\
\hline & 年 & 24.80250 & -167.17280 & 900.7 & & 13.4 & \\
\hline & 80. & 24.79820 & -167.16140 & 93 & & 1801 & \\
\hline 93 & $\begin{array}{l}0 \\
0118.54 \\
0.54\end{array}$ & 24.78970 & -167.13840 & 1289.2 & 53.8 & 163.4 & \\
\hline & 0128.54 & 24.78170 & -167.11560 & 1824.7 & .3 & & \\
\hline & 01 & 24.77800 & -167.10410 & 2016.0 & & $172-2>$ & \\
\hline & it & 77040 & $\begin{aligned}-167.0 \\
-167.0\end{aligned}$ & 2400.7 & & & \\
\hline $\begin{array}{l}293 \\
293\end{array}$ & $\begin{array}{l}0148.54 \\
01535.54\end{array}$ & 24.76640 & $\begin{array}{l}-167.06970 \\
-167\end{array}$ & $\begin{array}{l}2547.7 \\
2670.0\end{array}$ & 56.0 & 91.3 & \\
\hline & 01 & $\begin{array}{l}24.75810 \\
24.7524\end{array}$ & $\begin{array}{l}-167.04580 \\
-166703440\end{array}$ & 2791 & $\begin{aligned} 94.8 \\
115.5\end{aligned}$ & 80.5 & \\
\hline$<93$ & 6208.54 & 24.75050 & -167.023330 & 2836.5 & 129.3 & 06.1 & \\
\hline & 8.54 & 24.74250 & $7: 00080$ & 3015.7 & & & \\
\hline & 3.54 & 24.73790 & $\begin{array}{l}-160.98990 \\
-1600\end{array}$ & (3092.2. & & & \\
\hline & 3.54 & 24.72940 & $\begin{array}{l}-160.96040 \\
\end{array}$ & 3378.7 & 105.7 & & \\
\hline & 3 & .72580 & -166.95450 & 3428.2 & 94.4. & & \\
\hline & & & -160.93120 & & & & \\
\hline & 3.54 & 24.71400 & $-166.9 \mathrm{~g}$ & 3657.0 & 85.8 & 35.3 & \\
\hline 293 & 0303.54 & 24.70510 & -106.89470 & 3210.0 & 102.7 & $\begin{array}{l}30.2 \\
35.3\end{array}$ & 56.3 \\
\hline & $\begin{array}{l}8.5 \\
3.5\end{array}$ & 24.09520 & $\begin{array}{l}-1060.86060 \\
-16060\end{array}$ & $\begin{array}{l}32165.37 \\
3465\end{array}$ & 70.4 & 24.2 & \\
\hline & & 24.09030 & -160 & 3651.7 & & & \\
\hline 293 & $\begin{array}{l}032 \\
032\end{array}$ & 24.68 & $\begin{array}{l}-100.8 \\
-160.8\end{array}$ & 3739.5 & -22.2 & 10.2 & \\
\hline & 0338.54 & $\begin{array}{l}\begin{array}{l}24.65720 \\
24.07020\end{array}\end{array}$ & $\begin{array}{l}-1666.8 \\
106.7\end{array}$ & & & & \\
\hline & 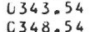 & $\begin{array}{l}66020 \\
60020\end{array}$ & $\begin{array}{l}-166.78340 \\
-160.70950\end{array}$ & $\begin{array}{l}3646.5 \\
3600.0\end{array}$ & & & \\
\hline 0 & $\begin{array}{l}0353.54 \\
03538.54\end{array}$ & $\begin{array}{l}24.65480 \\
24.09930\end{array}$ & $\begin{array}{l}-160.75 \\
-160.76 \\
-160\end{array}$ & 3654.8 & .2 & -9 & 41.8 \\
\hline 9.93 & 0.60 .56 & 24.04390 & -160.7 & 3762.0 & 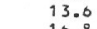 & -1 & \\
\hline & 4613.54 & 2 & -160.69890 & & & & \\
\hline 293 & $\begin{array}{l}0423 \\
042\end{array}$ & $\begin{array}{l}24.028700 \\
24.62670\end{array}$ & -166.67010 & $\begin{array}{l}4338.7 \\
19\end{array}$ & 17.5 & $\begin{array}{l}-28.8 \\
-28.0 \\
-10.8\end{array}$ & \\
\hline & & $\begin{array}{l}24.067380 \\
24.0780\end{array}$ & 160. & & & & \\
\hline & & 24.60190 & & 493 & .8 & & \\
\hline 93 & 0448.54 & $\begin{array}{l}24.5640 \\
24.59110\end{array}$ & $\begin{array}{l}-100.59690 \\
-160.590\end{array}$ & 5073.8 & $\begin{array}{l}4.4 \\
0.4 \\
4\end{array}$ & $\begin{array}{l}-49.2 \\
-49.4\end{array}$ & \\
\hline & & & & & & & \\
\hline 93 & & 急 & 80 & $\begin{array}{l}\begin{array}{l}5009.3 \\
50340\end{array} \\
\end{array}$ & $\begin{array}{l}-38.8 \\
-47.1 \\
-47.1\end{array}$ & $\begin{array}{l}-54.9 \\
-53.2 \\
-23\end{array}$ & \\
\hline & & $\begin{array}{l}24.56330 \\
2.5650\end{array}$ & & & & & \\
\hline & & 24.55080 & & & & & \\
\hline & & & & & & & \\
\hline & & & & & & & \\
\hline & 0543. & & & & & & \\
\hline 93 & 0553.54 & 24.50340 & -166.42140 & 4793.3 & 74.1 & $\begin{array}{l}-40.7 \\
-46.5 \\
\end{array}$ & 3. \\
\hline & & . 4886 & & 470 & & & \\
\hline & & & & & & & \\
\hline & & & & & & & \\
\hline & & $\begin{array}{l}4.5530 \\
46730\end{array}$ & & & & & \\
\hline & & 24.43960 & 31950 & 468 & -1585 & & \\
\hline & & & & & $2^{2}$ & s & \\
\hline & & $24: 0$ & $\begin{array}{l}-166 \\
-166\end{array}$ & 649 & & & \\
\hline & 003.54 & 24.39380 & 170 & 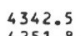 & 05 & 5.0 & \\
\hline & 08.54 & 24.36000 & -166.22830 & 4251.8 & & 0.8 & \\
\hline
\end{tabular}


TABLE 3 - Continued

\begin{tabular}{|c|c|c|c|c|c|c|c|}
\hline Day & Time & Latitude & Longitude & $\begin{array}{c}\mathrm{DEPTTH} \\
(\mathrm{m})\end{array}$ & $\underset{\substack{\text { MaG ANOM } \\
\text { (gmmas) }}}{ }$ & 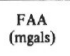 & $\begin{array}{c}\text { BGA } \\
\text { (mgals) }\end{array}$ \\
\hline 293 & $\begin{array}{l}9713.54 \\
07118.54\end{array}$ & $\begin{array}{l}24.37980 \\
26.37290\end{array}$ & $\begin{array}{l}-160.21460 \\
-160.20080\end{array}$ & $\begin{array}{l}4128.0 \\
3935.3\end{array}$ & $\begin{array}{l}-183.6 \\
-193.5\end{array}$ & 7 & $\begin{array}{l}292.1 \\
288.0\end{array}$ \\
\hline $\begin{array}{l}293 \\
293 \\
293\end{array}$ & $\begin{array}{l}07233.54 \\
0728.54 \\
0.725\end{array}$ & $\begin{array}{l}24.36010 \\
24.35930\end{array}$ & 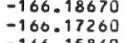 & $\begin{array}{l}374.0 \\
3553.5 \\
3.5\end{array}$ & $\begin{array}{l}-220.5 \\
-246.2 \\
-246.2\end{array}$ & $\begin{array}{l}25 \\
36\end{array}$ & $\begin{array}{l}283.7 \\
280.8 \\
280.8\end{array}$ \\
\hline & 0738.54 & & $\begin{array}{l}-1666.18860 \\
-160.04500\end{array}$ & $\begin{array}{l}\begin{array}{l}3290.3 \\
3008.3\end{array} \\
3\end{array}$ & & & $\begin{array}{l}273.7 \\
269: 0\end{array}$ \\
\hline 293 & $\begin{array}{l}0743.54 \\
074.58\end{array}$ & $\begin{array}{l}24.33930 \\
24.3150\end{array}$ & $\begin{array}{l}-660.13130 \\
-1160.118181\end{array}$ & $\begin{aligned} 2604.8 \\
2115.8\end{aligned}$ & $\begin{array}{c}-132.7 \\
-69.3 \\
-69.3\end{array}$ & $\begin{array}{r}80.8 \\
101.8\end{array}$ & $\begin{array}{l}260.2 \\
247.5\end{array}$ \\
\hline & $\begin{array}{l}0753.54 \\
0758.54\end{array}$ & $\begin{array}{l}24.32380 \\
24.31590\end{array}$ & $\begin{array}{l}-106.10500 \\
-106000200\end{array}$ & & & 125 & \\
\hline $\begin{array}{ll}2293 \\
2293\end{array}$ & 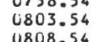 & $\begin{array}{l}24.310790 \\
24.30090\end{array}$ & $\begin{array}{l}-160.07890 \\
-\quad-166.0780\end{array}$ & 844.5 & $\begin{array}{l}-20.3 \\
52.5 \\
11.9\end{array}$ & 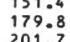 & $\begin{array}{l}238.0 \\
25.0\end{array}$ \\
\hline & 0813.54 & 24.29210 & $\begin{array}{l}-166.05270 \\
\end{array}$ & 705.0 & 25 & $\begin{array}{l}21 \\
21\end{array}$ & 262.4 \\
\hline 293 & $\begin{array}{l}0818.54 \\
0823.54\end{array}$ & $\begin{array}{l}24.288410 \\
24.27590\end{array}$ & $\begin{array}{l}-16060.03960 \\
-1606.02650\end{array}$ & $\begin{array}{l}699.8 \\
797.2 \\
\end{array}$ & $\begin{array}{l}-194.5 \\
-4355: 0 \\
-40.5\end{array}$ & $\begin{array}{l}220.4 \\
221.4\end{array}$ & $\begin{array}{l}\begin{array}{l}268.6 \\
276.3\end{array} \\
276.3\end{array}$ \\
\hline & $\begin{array}{l}0828.54 \\
0833.54\end{array}$ & $\begin{array}{l}24.26700 \\
24.25830\end{array}$ & $\begin{array}{l}-1660.01340 \\
-160.00100\end{array}$ & $\begin{array}{l}782.3 \\
752.3\end{array}$ & $\begin{array}{l}-413.6 \\
-220.0\end{array}$ & $\begin{array}{l}215 . \\
196 .\end{array}$ & \\
\hline & $\begin{array}{l}8.54 \\
3.54\end{array}$ & 24660 & -165.99210 & 828.7 & $\begin{array}{r}-170.8 \\
-0130.8\end{array}$ & $2000^{\circ}$ & 263.9 \\
\hline & 0848.54 & 24.22220 & $\begin{array}{l}-16559.98890 \\
-1655\end{array}$ & $\begin{array}{l}1056.7 \\
1344.7\end{array}$ & $\begin{array}{l}-134.8 \\
-76.3\end{array}$ & 1793. & $\begin{array}{l}206.5 \\
206.9\end{array}$ \\
\hline & & $\begin{array}{l}290400 \\
19680\end{array}$ & $\begin{array}{l}1065.96550 \\
-165095670\end{array}$ & 1600.5 & $\begin{array}{r}9.4 \\
113.7\end{array}$ & 158.8 & 269.0 \\
\hline $\begin{array}{l}29 \\
29\end{array}$ & $\begin{array}{l}0903.54 \\
09088.54\end{array}$ & $\begin{array}{l}24.18430 \\
24 \\
247180\end{array}$ & $\begin{array}{l}-165.96780 \\
-1665998890\end{array}$ & $\begin{array}{l}1962.0 \\
2198.02\end{array}$ & $\begin{array}{l}187.2 \\
263.7\end{array}$ & $\begin{array}{l}131 \\
121\end{array}$ & $\begin{array}{r}266.9 \\
272.5\end{array}$ \\
\hline & $\begin{array}{l}13.56 \\
13.56\end{array}$ & 24.15890 & $\begin{array}{l}-105.92990 \\
-1059\end{array}$ & & & & 279.6 \\
\hline & 3.54 & 4.13290 & -165.91150 & 2760.5 & 44 & & $\begin{array}{l}292 \\
298\end{array}$ \\
\hline & $\begin{array}{l}0.54 \\
.354\end{array}$ & $\begin{array}{l}10970 \\
10660\end{array}$ & -165.90180 & $\begin{array}{l}27339.8 \\
2737 \\
27\end{array}$ & & & 99.8 \\
\hline & & & -105.88280 & & 39 & & \\
\hline & 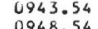 & 24.08060 & 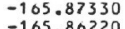 & $\begin{array}{l}25604.3 \\
2519\end{array}$ & $\begin{array}{l}326.6 \\
323\end{array}$ & & 3.2 \\
\hline 293 & 0953.54 & 24.05540 & -165.85100 & 2365.5 & 145.5 & 141 & 30 \\
\hline & $\begin{array}{l}1003.54 \\
\end{array}$ & 24.03060 & -105.83030 & 120.5 & & & 255.0 \\
\hline & $\begin{array}{l}10008.54 \\
1013\end{array}$ & 01820 & -165.82090 & & -7 & & 257. \\
\hline & & & -105.80250 & 856.5 & $\begin{array}{l}-196.7 \\
\end{array}$ & & \\
\hline $\begin{array}{l}293 \\
293\end{array}$ & $\begin{array}{l}1023.54 \\
1028\end{array}$ & $\begin{array}{l}23.98050 \\
23.9605\end{array}$ & $\begin{array}{l}-165.79330 \\
-1065\end{array}$ & $\begin{array}{l}720.0 \\
538.5\end{array}$ & $\begin{array}{r}-155.3 \\
-87.6\end{array}$ & $\begin{array}{l}2140^{2} \\
2280^{\circ}\end{array}$ & $\begin{array}{l}264.3 \\
265.2\end{array}$ \\
\hline & 1033.54 & 23.95720 & -165.77580 & 448.5 & $\begin{array}{l}-91.2 \\
-853\end{array}$ & 233. & 266.4 \\
\hline & & & & 360.5 & & & $\begin{array}{l}278.5 \\
288.5\end{array}$ \\
\hline 293 & $\begin{array}{l}1048.54 \\
1053.54\end{array}$ & $\begin{array}{l}23.92250 \\
23.91900\end{array}$ & $\begin{array}{l}-105574880 \\
-1065.79830\end{array}$ & $\begin{array}{l}317.3 \\
325.5\end{array}$ & $\begin{array}{l}469.7 \\
679.1\end{array}$ & $\begin{array}{l}288 . \\
280^{\circ}\end{array}$ & 309.3 \\
\hline 293 & $\begin{array}{l}1058.54 \\
110.54\end{array}$ & $\begin{array}{l}23.89930 \\
23.88750\end{array}$ & -165.73010 & 53,2 & 501.2 & $\begin{array}{l}292 . \\
292 .\end{array}$ & \\
\hline & 110 & 23.87600 & -165.71190 & 300 & -276.9 & & 15.7 \\
\hline & & 86420 & -165.73320 & 0.5 & & & \\
\hline & 1123.54 & 23.84050 & -105.06580 & 859.5 & -489.6 & & 31007 \\
\hline $\begin{array}{ll}29 \\
29\end{array}$ & $\begin{array}{l}1128.54 \\
1133.54\end{array}$ & $\begin{array}{l}23.82970 \\
23.81810\end{array}$ & $\begin{array}{l}-165.67700 \\
-1\end{array}$ & $\begin{array}{r}947.2 \\
1035.7\end{array}$ & $\begin{array}{l}-464.3 \\
-335.3\end{array}$ & $\begin{array}{l}236 . \\
223 .\end{array}$ & $\begin{array}{l}301.3 \\
294.7\end{array}$ \\
\hline 29 & $\begin{array}{l}1138.54 \\
1143.54\end{array}$ & $\begin{array}{l}23.80630 \\
23.7940\end{array}$ & $\begin{array}{l}-165.65900 \\
-1065005030\end{array}$ & $\begin{array}{l}1269.7 \\
1478.2\end{array}$ & -264.8 & 207.8 & 295.2 \\
\hline 293 & 1146.54 & 23.78260 & -165.04150 & 1460.8 & -30.7 & 185.1 & 年 285.8 \\
\hline & 1158.54 & 23.75870 & -105.02450 & & & & \\
\hline C9 & 1248.54 & $\begin{array}{l}23.74600 \\
23.73430\end{array}$ & $\begin{array}{l}1055.061620 \\
-165.00780\end{array}$ & $\begin{array}{l}11780.0 \\
1254: 0\end{array}$ & $\begin{array}{r}-55.9 \\
-112.2\end{array}$ & $\begin{array}{l}176 \\
173\end{array}$ & 59. \\
\hline & & $\begin{array}{l}23.72190 \\
23.70960\end{array}$ & $\begin{array}{l}1605.59950 \\
-165.59100\end{array}$ & & -331 & & \\
\hline & 1223.54 & & & 11.7 & -196.0 & & 5.8 \\
\hline 293 & 1233.54 & 23.073100 & $\begin{array}{l}10050 \\
-105.50620\end{array}$ & 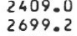 & $\begin{array}{l}12.5 \\
70.2\end{array}$ & $\begin{array}{l}215.0 \\
113.1\end{array}$ & $\begin{array}{l}290.9 \\
299.0\end{array}$ \\
\hline & 1243.54 & 23.04850 & $\begin{array}{l}-16 \\
-16\end{array}$ & 3005.2 & $\begin{array}{r}35.4 \\
0.6\end{array}$ & & 289.3 \\
\hline $\begin{array}{l}293 \\
293 \\
293\end{array}$ & $\begin{array}{l}1248.54 \\
1253.54\end{array}$ & $\begin{array}{l}23.63020 \\
23.02300\end{array}$ & $\begin{array}{l}-1655.54140 \\
-105535270\end{array}$ & $\begin{array}{l}33461.2 \\
3480: 0\end{array}$ & $\begin{array}{l}-25.0 \\
-46.3\end{array}$ & i: & 301.0 \\
\hline & $\begin{array}{l}12568.54 \\
1303\end{array}$ & $\begin{array}{l}23.61100 \\
23.59900\end{array}$ & $\begin{array}{l}-165.52410 \\
-1055551490\end{array}$ & 519,8 & $\begin{array}{l}-57.3 \\
-540\end{array}$ & & \\
\hline & & 30 & & 690 & -4 & & 290.5 \\
\hline & $\begin{array}{l}1313.54 \\
131.54\end{array}$ & 23.57570 & -105.49600 & $\begin{array}{l}3588.7 \\
3728\end{array}$ & -49.0 & & 300 \\
\hline 29 & 1323.54 & 23.55370 & -165.47670 & 3817.5 & -51.7 & 30 & 29 \\
\hline & $\begin{array}{l}.54 \\
.54\end{array}$ & 23.56280 & $\begin{array}{l}-105.4040450 \\
\end{array}$ & & -62 & & \\
\hline & $\begin{array}{l}13.3 \\
134\end{array}$ & 23.56750 & -165.42800 & 1.2 & -69 & & 01 \\
\hline 29 & 1348.54 & 23.57580 & -165.39520 & 4048.5 & -80.5 & 20.3 & 294.1 \\
\hline & & 23.58380 & $\cdots$ & & & & 296.0 \\
\hline 293 & $\begin{array}{l}14008584 \\
140854\end{array}$ & $\begin{array}{l}23.58540 \\
23.58700\end{array}$ & $\begin{array}{l}-165.3 \\
-165.3 \\
-\end{array}$ & $\begin{array}{r}\begin{array}{r}4022.3 \\
4020.0\end{array} \\
4\end{array}$ & $\begin{array}{l}-94.4 \\
-96.7\end{array}$ & $\begin{array}{l}11.8 \\
12.7\end{array}$ & 288.8 \\
\hline & $\begin{array}{l}1413.54 \\
1418.54\end{array}$ & $\begin{array}{l}23.58700 \\
23.58800\end{array}$ & $\begin{array}{l}-165531130 \\
-105: 30210\end{array}$ & $\begin{array}{l}4020.0 \\
600200\end{array}$ & $\begin{array}{l}-90 \\
-74\end{array}$ & $\begin{array}{l}15 \\
15 \\
15\end{array}$ & 2020 \\
\hline & & & 65.28 & & & & \\
\hline & $\begin{array}{l}148.54 \\
143.54\end{array}$ & $\begin{array}{r}23559150 \\
235950\end{array}$ & $\begin{array}{l}-1 \\
-155\end{array}$ & & $\begin{array}{l}-45 \\
-430\end{array}$ & & \\
\hline & & 23.342 & & & & & \\
\hline & 144 & $\begin{array}{l}23.59990 \\
23.5990\end{array}$ & $\begin{array}{r}-165.22 \\
-105\end{array}$ & & -19.0 & & \\
\hline 29 & & 23.60040 & -165.18860 & 3665.3 & 2. & & \\
\hline & & 23.60390 & & 517 & & & \\
\hline $\begin{array}{l}29 \\
29 \\
29\end{array}$ & 54 & $\begin{array}{l}23.64550 \\
23.60770\end{array}$ & $\begin{array}{l}-16551 \\
-165: 12\end{array}$ & & $\begin{array}{l}-10.9 \\
-25.6\end{array}$ & $\begin{array}{l}84.6 \\
53.7\end{array}$ & \\
\hline & & $\begin{array}{l}23.61 \\
23.61\end{array}$ & & & & & $\begin{aligned} 287.8 \\
289.8\end{aligned}$ \\
\hline & & & 65. & & & & \\
\hline 29 & & $\begin{array}{l}23.6 \\
23.6\end{array}$ & $\begin{array}{l}-165 \\
-105 \\
-105\end{array}$ & $\begin{array}{l}2776.5 \\
2610.8\end{array}$ & $\begin{array}{l}28 . \\
-0 .\end{array}$ & & \\
\hline & & $\begin{array}{l}23.6 \\
23.6\end{array}$ & $\begin{array}{c}-105 \\
1055 \\
105\end{array}$ & & 37 & $\begin{array}{l}109 . \\
1124\end{array}$ & 278. \\
\hline & & $\begin{array}{l}23.622 \\
23.62\end{array}$ & $\begin{array}{l}-164.99 \\
-16498\end{array}$ & & & & \\
\hline & 54 & 23.62490 & -104.90040 & 1061.3 & 26 & 184 & \\
\hline & & & & & & & \\
\hline & $\begin{array}{l}1018 \\
1023\end{array}$ & $\begin{array}{l}23.6 \\
23.6\end{array}$ & $\begin{array}{l}-104.5 \\
-164.9\end{array}$ & & 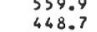 & & \\
\hline 293 & $\begin{array}{l}1628.54 \\
1633.54\end{array}$ & $\begin{array}{l}23.03650 \\
23.64210\end{array}$ & $\begin{array}{r}166488890 \\
-164.87390\end{array}$ & $\begin{array}{l}3994.5 \\
390: 0\end{array}$ & $\begin{array}{r}10.2 \\
-280.0\end{array}$ & $\begin{array}{l}520.7 \\
248.8\end{array}$ & 5.7 \\
\hline
\end{tabular}

TABLE 3 - Continued

\begin{tabular}{|c|c|c|c|c|c|c|c|}
\hline Day & Time & Latitude & Longitude & 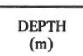 & 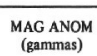 & 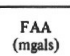 & $\begin{array}{c}\mathrm{BGA} \\
\text { (mgals) }\end{array}$ \\
\hline 293 & 1038.54 & $\begin{array}{l}23.04750 \\
23.65210\end{array}$ & -106.85770 & $\begin{array}{l}405.7 \\
550.9\end{array}$ & $\begin{array}{l}-297.6 \\
-3461\end{array}$ & 239.0 & 266.9 \\
\hline 26 & $\begin{array}{l}1643.54 \\
1648.54\end{array}$ & $\begin{array}{l}23.652210 \\
23.65030\end{array}$ & $\begin{array}{l}-164.8460 \\
-164.82570\end{array}$ & $\begin{array}{l}5559.5 \\
837.7\end{array}$ & $\begin{array}{l}-341.1 \\
-355: .8\end{array}$ & $\begin{array}{l}225.3 \\
208.1\end{array}$ & \\
\hline & $\begin{array}{l}3.54 \\
8.54\end{array}$ & $\begin{array}{l}23.66030 \\
23.6635\end{array}$ & $\begin{array}{l}-164.80880 \\
-1640\end{array}$ & & $\begin{array}{r}-234.8 \\
-55.8\end{array}$ & 209.0 & \\
\hline & 1703.54 & $\begin{array}{r}23.06770 \\
\end{array}$ & -166.72650 & 1682.2 & 82.7 & & 275.7 \\
\hline 293 & $\begin{array}{l}17808.54 \\
1713.54\end{array}$ & $\begin{array}{l}23.67210 \\
23.67610\end{array}$ & $\begin{array}{l}-164.75990 \\
-164.74370\end{array}$ & $\begin{array}{l}\frac{1551.8}{1456.5} \\
1,56\end{array}$ & $\begin{array}{l}148.7 \\
109.7\end{array}$ & $\begin{array}{l}174.2 \\
179.1\end{array}$ & \\
\hline & $\begin{array}{l}1718.54 \\
1723.54\end{array}$ & $\begin{array}{l}23.68000 \\
23.68400\end{array}$ & $\begin{array}{r}-104.7250 \\
-164.71120\end{array}$ & $\begin{array}{l}11227.3 \\
743.3\end{array}$ & $\begin{array}{r}-329.1 \\
-226.2\end{array}$ & $\begin{array}{l}1887.5 \\
201.7\end{array}$ & $\begin{array}{l}265.1 \\
252.9\end{array}$ \\
\hline & & $\begin{array}{l}23.68800 \\
23\end{array}$ & $\begin{array}{l}-164.69500 \\
-16467888\end{array}$ & $\begin{array}{l}579.8 \\
439.5\end{array}$ & $\begin{array}{l}-375.5 \\
-32.59\end{array}$ & & $\begin{array}{l}255.0 \\
256.1\end{array}$ \\
\hline 293 & 1738.54 & 23.69460 & -104.60220 & 390.0 & -323.6 & 236.2 & $\begin{array}{l}256 \\
263\end{array}$ \\
\hline $\begin{array}{l}293 \\
293\end{array}$ & $\begin{array}{l}1743.54 \\
1748.54\end{array}$ & $\begin{array}{l}23.697700 \\
23.69950\end{array}$ & $\begin{array}{r}-1040.64500 \\
-164.02890\end{array}$ & $\begin{array}{l}\begin{array}{l}374.3 \\
346.5\end{array} \\
340\end{array}$ & $\begin{array}{l}-414.0 \\
-467.9\end{array}$ & $\begin{array}{l}244.5 \\
247.3\end{array}$ & \\
\hline & $\begin{array}{l}1753.54 \\
1758.54\end{array}$ & $\begin{array}{l}23.70200 \\
23.7040\end{array}$ & & & & & \\
\hline $\begin{array}{l}293 \\
293 \\
293\end{array}$ & $\begin{array}{l}1803.54 \\
1808.54\end{array}$ & $\begin{array}{l}23.70790 \\
2371190\end{array}$ & $\begin{aligned}-104.57940 \\
-1640450550\end{aligned}$ & 307.5 & -201.1 & 279.4 & \\
\hline $\begin{array}{l}293 \\
293\end{array}$ & $\begin{array}{l}1813.54 \\
1818.54\end{array}$ & $\begin{array}{l}23.71550 \\
23.7190\end{array}$ & $\begin{array}{l}-164.547070 \\
-164053200\end{array}$ & $\begin{array}{l}305.2 \\
312.7\end{array}$ & & $\begin{array}{l}2551.6 \\
257.4\end{array}$ & 27 \\
\hline $\begin{array}{l}293 \\
293\end{array}$ & $\begin{array}{l}18233.54 \\
1828.54\end{array}$ & $\begin{array}{l}23.723300 \\
23.72610\end{array}$ & $\begin{array}{r}-1045051020 \\
-164050010\end{array}$ & $\begin{array}{l}320.2 \\
327.7\end{array}$ & -59.0 & $\begin{array}{l}310.6 \\
307.6\end{array}$ & $\begin{array}{l}332.7 \\
330.2\end{array}$ \\
\hline 293 & 1838.54 & $\begin{array}{l}23.72900 \\
23.73240\end{array}$ & $\begin{array}{r}-104.48880 \\
-166.460760\end{array}$ & 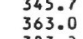 & & $\begin{array}{l}299.1 \\
282.8\end{array}$ & $\begin{array}{l}31 \\
30\end{array}$ \\
\hline & 1848.54 & 23.73990 & 4.43490 & 年 & -352.4 & 259.3 & \\
\hline & $\begin{array}{l}3.54 \\
3.54\end{array}$ & 23.74370 & $\begin{array}{l}-164.49600 \\
-140\end{array}$ & 588.7 & -362.5 & & \\
\hline & 40 & & & 160.0 & -538.2 & & \\
\hline & 8.54 & . 75530 & -164.37300 & 1238.2 & -639.1 & 193.0 & \\
\hline & 象.54 & T.280 & 40.34000 & $\begin{array}{l}2306.7 \\
2316.7\end{array}$ & -844.3 & 1002 & \\
\hline & 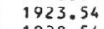 & . & 44.32450 & 2436.7 & -460.4 & & \\
\hline & & & & 237563 & & $\begin{array}{l}132.8 \\
1280\end{array}$ & \\
\hline & 1938.54 & 23.77800 & -164.27680 & 2306.3 & -179.6 & & \\
\hline $\begin{array}{l}293 \\
293 \\
293\end{array}$ & $\begin{array}{l}1943.54 \\
1948.54\end{array}$ & $\begin{array}{l}23.78320 \\
23.78780\end{array}$ & $\begin{array}{c}-164.260100 \\
-164.24520\end{array}$ & 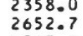 & $\begin{array}{l}-96.8 \\
-16.3\end{array}$ & $\begin{array}{l}116.0 \\
103.2\end{array}$ & \\
\hline & 8.54 & $\begin{array}{l}23.79240 \\
23.79070\end{array}$ & -164.21390 & & $\begin{array}{l}42.7 \\
78.7\end{array}$ & & \\
\hline & 3.54 & 23.80110 & -164.19850 & 3973.5 & 94.0 & 51.2 & 2.40 \\
\hline 293 & $\begin{array}{l}0.54 \\
3.54\end{array}$ & & & 4599.2 & (2).5 89.5 & & \\
\hline & & 23.80650 & -164.15120 & 4653.0 & 68.6 & 16.8 & \\
\hline $\begin{array}{l}293 \\
293\end{array}$ & $\begin{array}{l}20233.54 \\
2028.54\end{array}$ & $\begin{array}{l}23.80770 \\
23.8090\end{array}$ & $\begin{array}{r}-1640.13540 \\
-164.11960\end{array}$ & $\begin{array}{l}66622.7 \\
4680.7\end{array}$ & $\begin{array}{l}44.7 \\
25.3\end{array}$ & $\begin{array}{r}10.0 \\
3.5\end{array}$ & 325.8 \\
\hline (29 & $\begin{array}{l}2033.54 \\
2036.54\end{array}$ & $\begin{array}{l}23.81090 \\
23.81300\end{array}$ & $\begin{array}{l}-164.10400 \\
-16408050\end{array}$ & $\begin{array}{l}\begin{array}{l}66998.0 \\
4697.3\end{array} \\
4\end{array}$ & 1.4 & 5.2. & $\begin{array}{l}322.0^{\circ} \\
318 .\end{array}$ \\
\hline & 2043.54 & 23.81100 & -164.07860 & 4700.2 & -0.9 & -76.5 & \\
\hline $\begin{array}{l}293 \\
293 \\
293\end{array}$ & $\begin{array}{l}2048.54 \\
2053.54\end{array}$ & $\begin{array}{l}23.79910 \\
23.78720\end{array}$ & $\begin{array}{r}-166.08900 \\
-164.08520\end{array}$ & 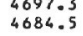 & $\begin{array}{r}60.4 \\
20.0\end{array}$ & $\begin{aligned}-23.0 \\
13.6 \\
13.6\end{aligned}$ & 336.2 \\
\hline & Sis & $\begin{array}{l}23.775530 \\
23.76340\end{array}$ & $\begin{array}{l}=104.04919910 \\
-164.0999\end{array}$ & 4650.0 & $\begin{array}{l}37.5 \\
58.2\end{array}$ & 3.7 & 23. \\
\hline 293 & 2108.54 & 23.75110 & -164.10620 & & 80.5 & & \\
\hline & 54 & $\begin{array}{l}23.738040 \\
32,7350\end{array}$ & $\begin{array}{l}-104 \cdot 11340 \\
-1720\end{array}$ & $\begin{array}{l}4601.3 \\
4658.8\end{array}$ & 70.4 & $18.98-3$ & \\
\hline & 4 & 23.71280 & -164.12780 & .3 & 81.6 & 25.6 & 312 \\
\hline 280 & $\begin{array}{l}2128.56 \\
21333 \\
21354\end{array}$ & $\begin{array}{l}23.69990 \\
23.6870\end{array}$ & $\begin{array}{l}-104.13520 \\
-1640419260\end{array}$ & $\begin{array}{l}3865.5 \\
3601.5 \\
3605\end{array}$ & $\begin{array}{r}38.8 \\
-19.2\end{array}$ & $\begin{array}{l}34.9 \\
45.2\end{array}$ & . \\
\hline $28 \mathrm{~g}$ & $\begin{array}{l}21388.54 \\
2\end{array}$ & $\begin{array}{l}23.67390 \\
23\end{array}$ & -164.14960 & $\begin{array}{l}3414.8 \\
324.8\end{array}$ & -898.7 & 56.8 & \\
\hline & 8.54 & 23. & -104.16380 & 3116.0 & -236.4 & 76.1 & \\
\hline . & $\begin{array}{l}21533.54 \\
2156.54\end{array}$ & $\begin{array}{l}23.033500 \\
23.61980\end{array}$ & -164.17890 & & $\begin{array}{l}-223.5 \\
-2123.5 \\
\end{array}$ & & \\
\hline & & 23.00030 & -164.18530 & 2677 & -91.6 & 110.6 & \\
\hline $\begin{array}{l}293 \\
293\end{array}$ & $\begin{array}{l}2208.54 \\
2213.54\end{array}$ & $\begin{array}{r}23.59280 \\
2335790\end{array}$ & $\begin{array}{l}-164 \cdot 19240 \\
-104: 19960\end{array}$ & 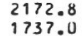 & $\begin{array}{l}-1616.3 \\
-298.6\end{array}$ & $\begin{array}{l}123.0 \\
137.3\end{array}$ & \\
\hline $\begin{array}{l}29 \\
29\end{array}$ & $\begin{array}{l}22183.54 \\
2223.54\end{array}$ & $\begin{array}{l}23.5650 \\
23.55100\end{array}$ & $\begin{array}{l}-104: 20080 \\
-164: 21400\end{array}$ & $\begin{array}{l}124.0 .5 \\
0.56 .5\end{array}$ & $\begin{array}{l}-380 \\
-437\end{array}$ & $\begin{array}{l}154.1 \\
1710\end{array}$ & \\
\hline & 54 & 23.53730 & -164.22120 & 805.5 & -521.5 & 187.5 & \\
\hline & $\begin{array}{l}2233.54 \\
2238\end{array}$ & $\begin{array}{l}23.52220 \\
23.5050\end{array}$ & 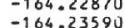 & $\begin{array}{l}6968.8 \\
6.25 .5\end{array}$ & $\begin{array}{l}-6090.2 \\
-592.5\end{array}$ & $\begin{array}{l}199.6 \\
2030\end{array}$ & 246.6 \\
\hline & & 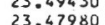 & -104.25 & & $\begin{array}{l}-517.0 \\
-492.1\end{array}$ & $\begin{array}{l}2210.2 \\
25.7\end{array}$ & \\
\hline 29. & 2253.54 & 23.4 & -164.25850 & 519.0 & $\begin{array}{l}-439.2 \\
-5,51.2\end{array}$ & 23 & \\
\hline 293 & 2303.54 & 23.43990 & -104.2 & 60.3 & & 261.9 & $290^{\circ}$ \\
\hline & 8.54 & & -104.27900 & & & 21 & \\
\hline & $\begin{array}{r}2318.54 \\
3323\end{array}$ & 23.40420 & $\begin{aligned}-104.29300 \\
-1\end{aligned}$ & 41.3 & & 296.2 & 299. \\
\hline 29 & $\begin{array}{l}2328.54 \\
323335\end{array}$ & 23.37690 & $-104 \cdot 30820$ & 30. & & 31 & \\
\hline & & & & & & & \\
\hline 2 & 54 & $\begin{array}{l}23.33 \\
23.33\end{array}$ & 0 & (37) 37 & & 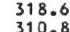 & \\
\hline 2 & & 23.30710 & -164334600 & 37: & & 300 & \\
\hline & & $\begin{array}{l}23.27 \\
23.27\end{array}$ & & 32. & & & \\
\hline & & 23.26 & 4.3 & & & & \\
\hline & & $\begin{array}{l}5.6 \\
23.2\end{array}$ & & 305.2 & & & 29 \\
\hline $\begin{array}{l}296 \\
2996\end{array}$ & $\begin{array}{l}002.58 \\
0025.54\end{array}$ & $\begin{array}{l}23.22000 \\
23.20900\end{array}$ & 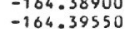 & 146.00 & & 108.8 & \\
\hline $\begin{array}{l}294 \\
294 \\
204\end{array}$ & $\begin{array}{l}0033.54 \\
00038.54\end{array}$ & $\begin{array}{l}23.19220 \\
23.17600\end{array}$ & $\begin{aligned}-104.40200 \\
-164.40800\end{aligned}$ & $\begin{array}{l}2127 \\
2551\end{array}$ & & $\begin{array}{c}15.0 \\
83.8\end{array}$ & 25 \\
\hline 294 & $\begin{array}{l}0043.54 \\
0068.54\end{array}$ & $\begin{array}{l}23.16500 \\
23.15160\end{array}$ & $\begin{array}{r}-164641520 \\
-164.42190\end{array}$ & 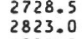 & $\begin{array}{l}206.6 \\
215.5\end{array}$ & $\begin{array}{l}117.3 \\
106.4\end{array}$ & \\
\hline & & 23. & & & & & \\
\hline & 0103.54 & $\begin{array}{l}23.10900 \\
{ }_{23}\end{array}$ & & $\begin{array}{l}3042.7 \\
3155.65\end{array}$ & 194.4 & $\begin{array}{l}67.5 \\
60.5\end{array}$ & 237 \\
\hline & 0118.54 & $\begin{array}{l}23.08490 \\
23\end{array}$ & & 3360.7 & 91.8 & $\begin{array}{r}52.9 \\
139.5\end{array}$ & 37 \\
\hline & 0130.04 & $\begin{array}{l}23.08090 \\
23.07900\end{array}$ & & 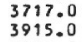 & 1 & & \\
\hline & & & & & & & \\
\hline $\begin{array}{l}2 \\
28 \\
28\end{array}$ & 0148.54 & 23.07300 & & $\begin{array}{l}3624.8 \\
304,8\end{array}$ & -29.0 & 30.6 & 283. \\
\hline & $\begin{array}{r}0158.54 \\
0203\end{array}$ & $\begin{array}{l}23.07080 \\
23.0690\end{array}$ & $\begin{array}{l}-104.36500 \\
-16403580\end{array}$ & 3710.2 & -710.4 & $\begin{array}{l}\begin{array}{l}31.3 \\
30.9\end{array} \\
30.9\end{array}$ & \\
\hline
\end{tabular}


PRE-LEG 55 SITE SURVEY GEOPHYSICAL DATA

TABLE 3 - Continued

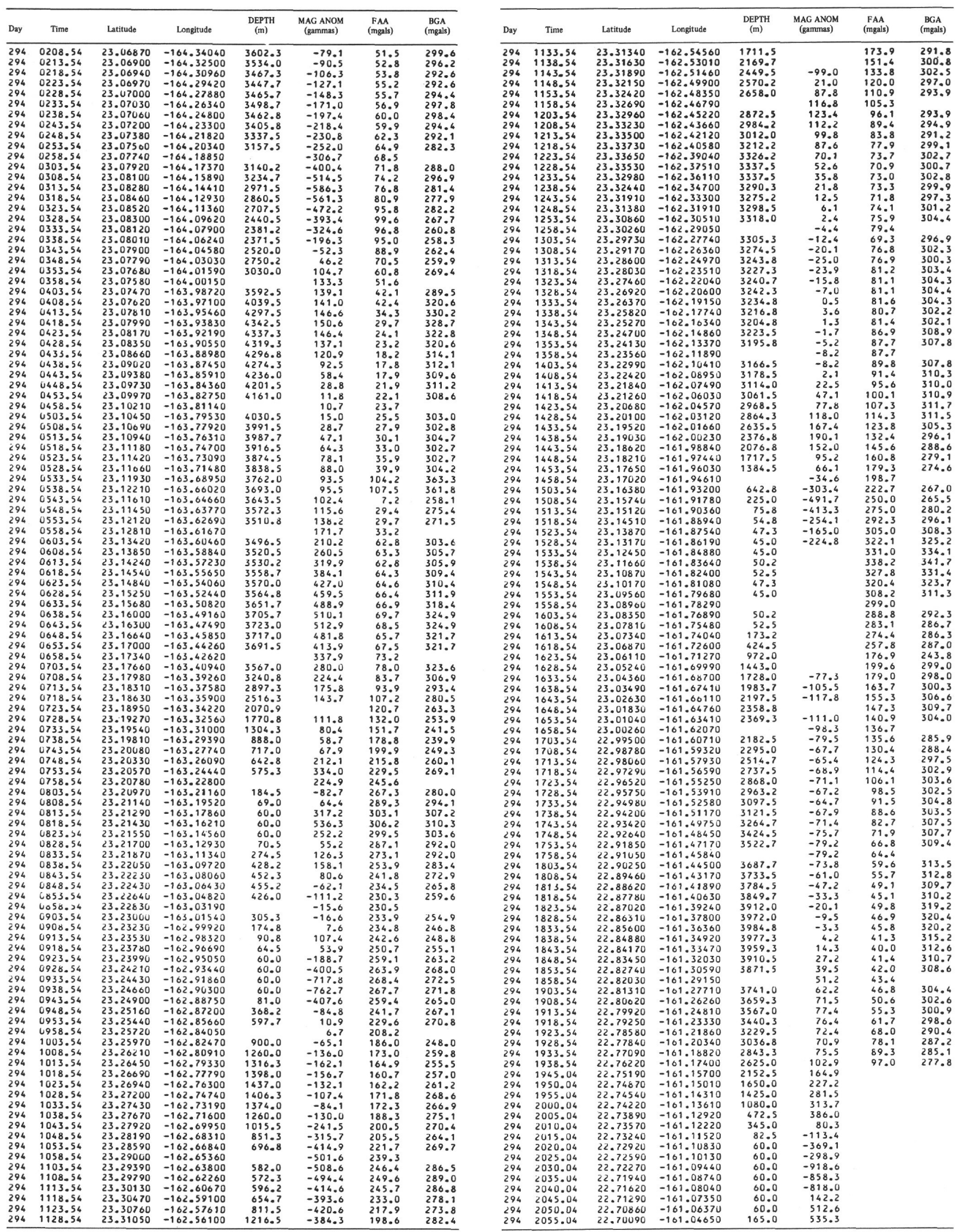

TABLE 3 - Continued 
TABLE 3 - Continued

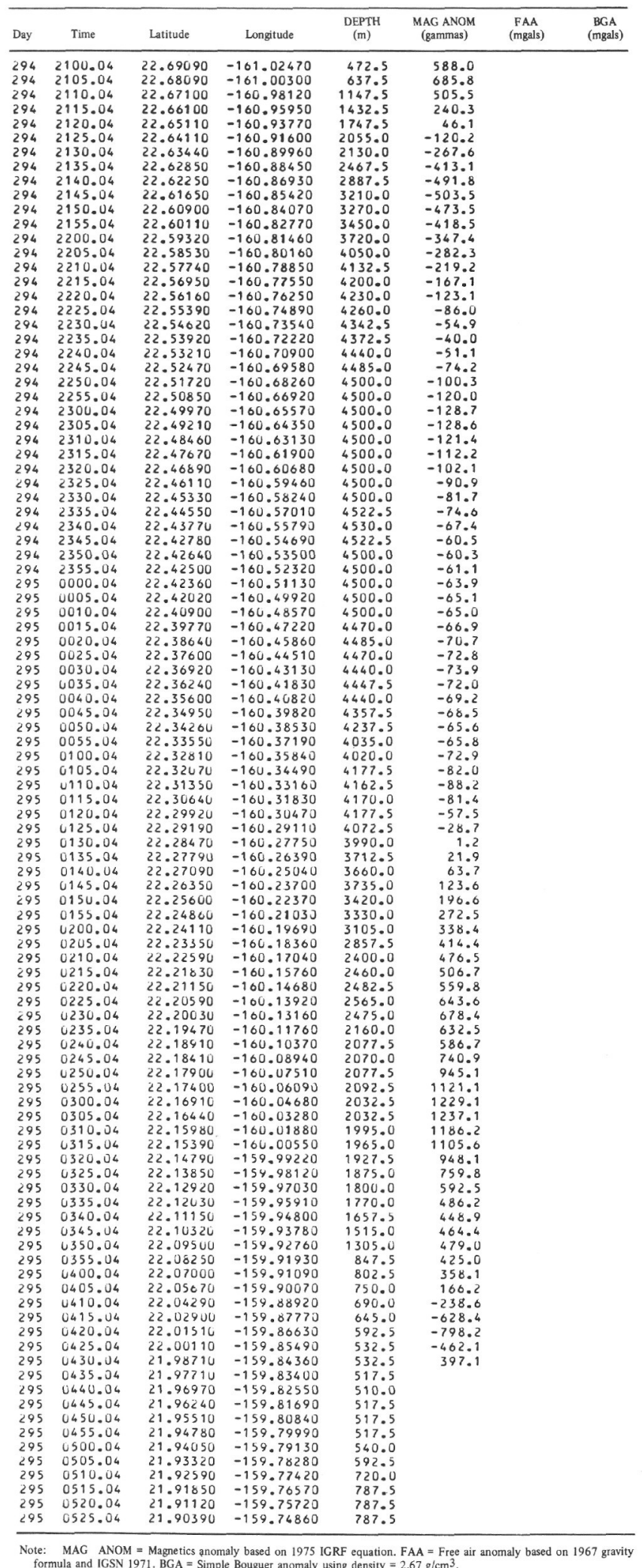

is probably over 200 meters. The reef sediments have maximum thicknesses ranging from 335 meters in the central part of the lagoon to around 170 meters on the fringes of the seamount, where constructional terrace deposits are located. The surface of the volcanic rocks is erosional and very irregular. This volcanic surface crops out on the west flank at a depth of 1575 meters and on the east flank at a depth of 1685 meters. A small, sharppeaked volcanic cone projects above Jingū's western flank. A considerable amount of slump detritus has accumulated along the lower eastern flank of the seamount.

The broad negative Bouguer anomaly reflects the sediment-filled, lagoon-reef structure.

\section{Kōkō Seamount}

Kōkō Seamount (Figure 12), is a large, flat-topped seamount located at $35^{\circ} \mathrm{N}$ latitude, $171^{\circ} 40^{\prime} \mathrm{E}$ longitude. It is $111 \mathrm{~km}(60 \mathrm{n}$. mi.) wide and $185 \mathrm{~km}(100 \mathrm{n}$. mi.) long, with a northwest-southeast elongation. The flat top of the seamount is about $10,500 \mathrm{~km}^{2}$ in area; its flat central part lies at a depth of 335 meters; the edge of the top surface is 850 meters deep on the north and 1125 meters deep on the south. The flanks are less steep than those of the seamounts to the north; inclinations are $9^{\circ}$ on the north and $7^{\circ}$ on the south. Elevation above the adjacent sea floor varies from 4725 meters on the north to 5290 meters on the south.

The seismic reflection profile indicates a complex reef morphology. The main part of the flat crestal area is covered with a layer of well-bedded reefal deposits about 200 meters thick. Included within and overlying these deposits are features that, acoustically and morphologically, appear to be reefs (Davies et al., 1972; Greene, this volume). Fringing reefs surround the central flat area of Kōkō and are found at depths of 785 meters on the north and 787 meters on the south. The southern fringing reef is extensive, over $20 \mathrm{~km}$ wide and nearly 150 meters thick. Terrace deposits occur on the edge of the seamount, principally outboard of the fringing reefs. About 200 meters of lagoonal deposits are concentrated in a structural depression on the south side of the seamount, inboard of the large, massive, fringing reef.

Faults are prominent in the seismic reflection profile, and several normal faults are concentrated in the depression on the south side of the seamount. These faults offset the volcanic surface and displace the overlying lagoonal deposits and reef rocks. A graben formed by two of these faults suggests that tension in this area, perhaps the result of structural downbowing of the depression, is the principal force at work. A large normal fault on the north side of the seamount offsets the volcanic surface nearly 400 meters, forming a steep subsurface scarp against which flat-lying terrace deposits abut. The north flank of the seamount is relatively free of slump detritus, compared with the more gentle south flank.

The residual magnetic anomaly profile across Kōkō is very irregular, and shows large negative and positive anomalies, one of which approaches 1000 gammas. These anomalies may be due in part to an undulating volcanic surface underlying a fairly thick sedimentary cover, but may also reflect magnetic inhomogeneities in the volcanic rocks. The large positive anomaly over the 

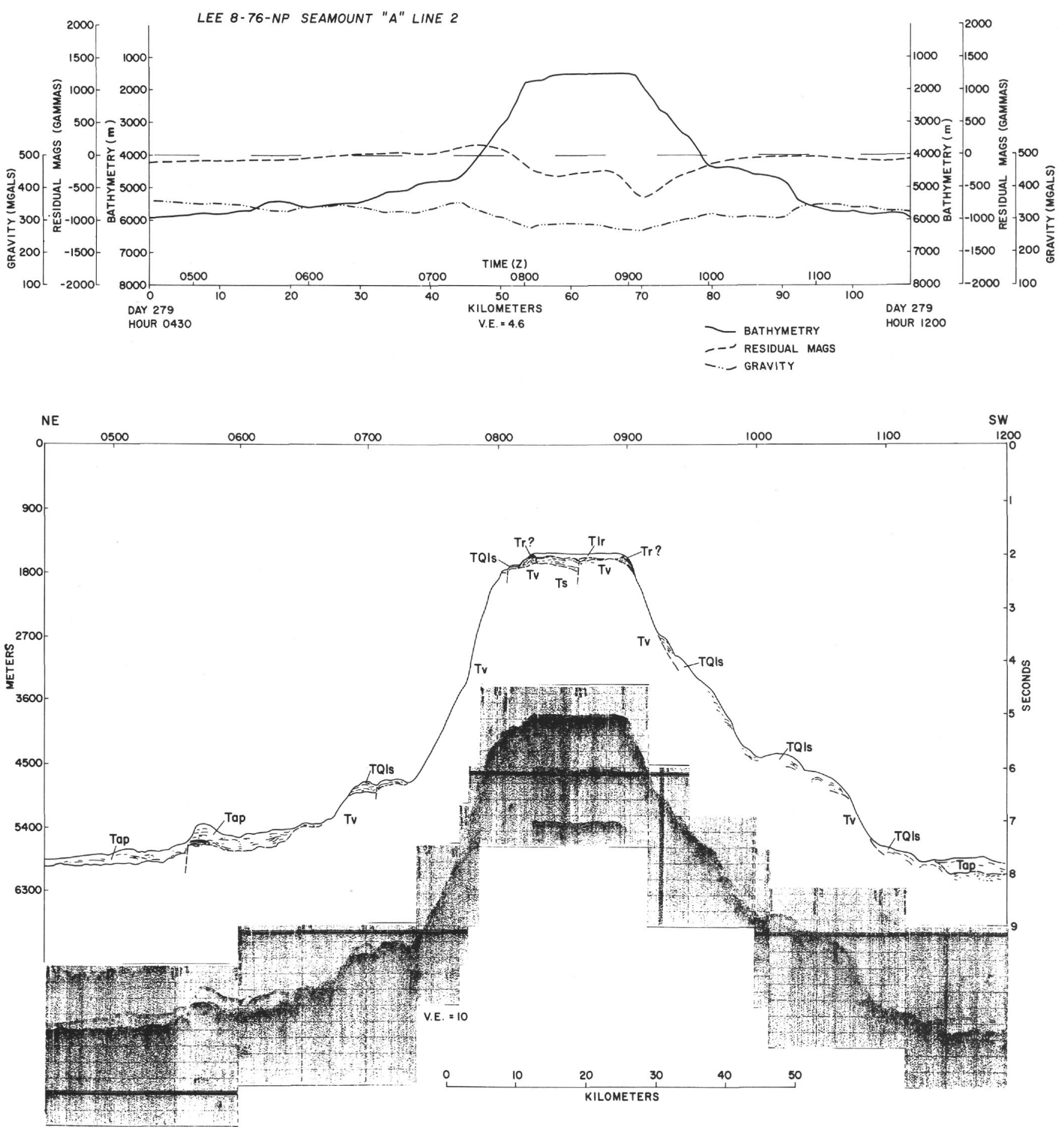

Figure 5. Geophysical profile across an unnamed seamount (Seamount " $A$ ") north of Suiko. Bathymetry is in uncorrected meters, calculated at a velocity of $1500 \mathrm{~m} / \mathrm{s}$. Residual magnetic anomaly with 1975 IGRF removed is shown as dashed line. Bouguer gravity anomaly shown by dash-dot line. Tv, Tertiary volcanic rocks (acoustic basement); Ts, Tertiary shallow-water bank or reef-flat deposits; Tlr, Tertiary back-reef or lagoonal sediments; Tr, Tertiary organic reef limestone; Tap, Tertiary pelagic sediments (acoustically transparent layer); TQls, Tertiary and Quaternary slump deposits. Location of profile shown in Figure 1. 

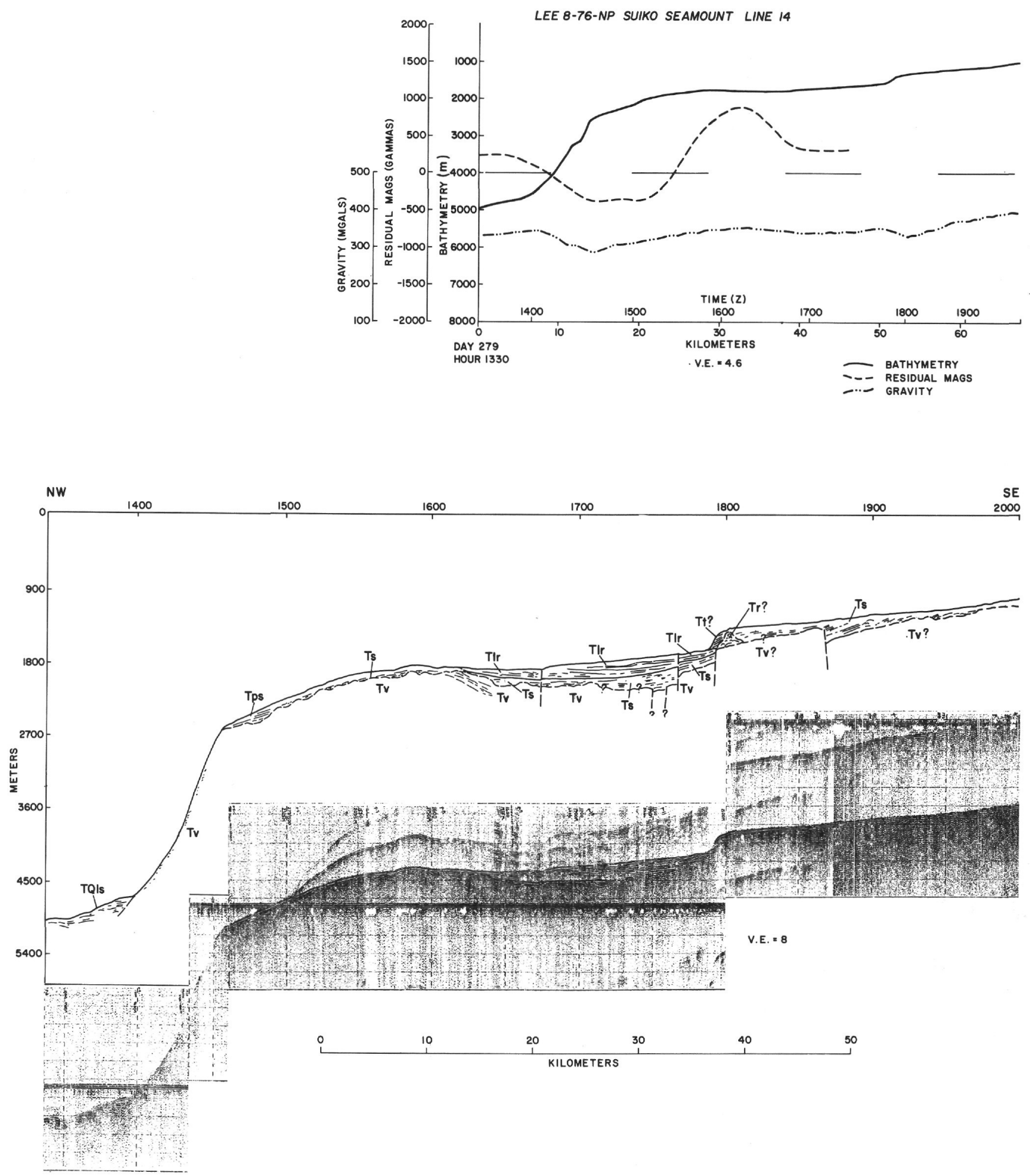

Figure 6. Geophysical profile across Suiko Seamount. Tps, Tertiary ponded sediments; Tt, Tertiary terrace deposits; other symbols as in Figure 5. Location of profile shown in Figure 1. 

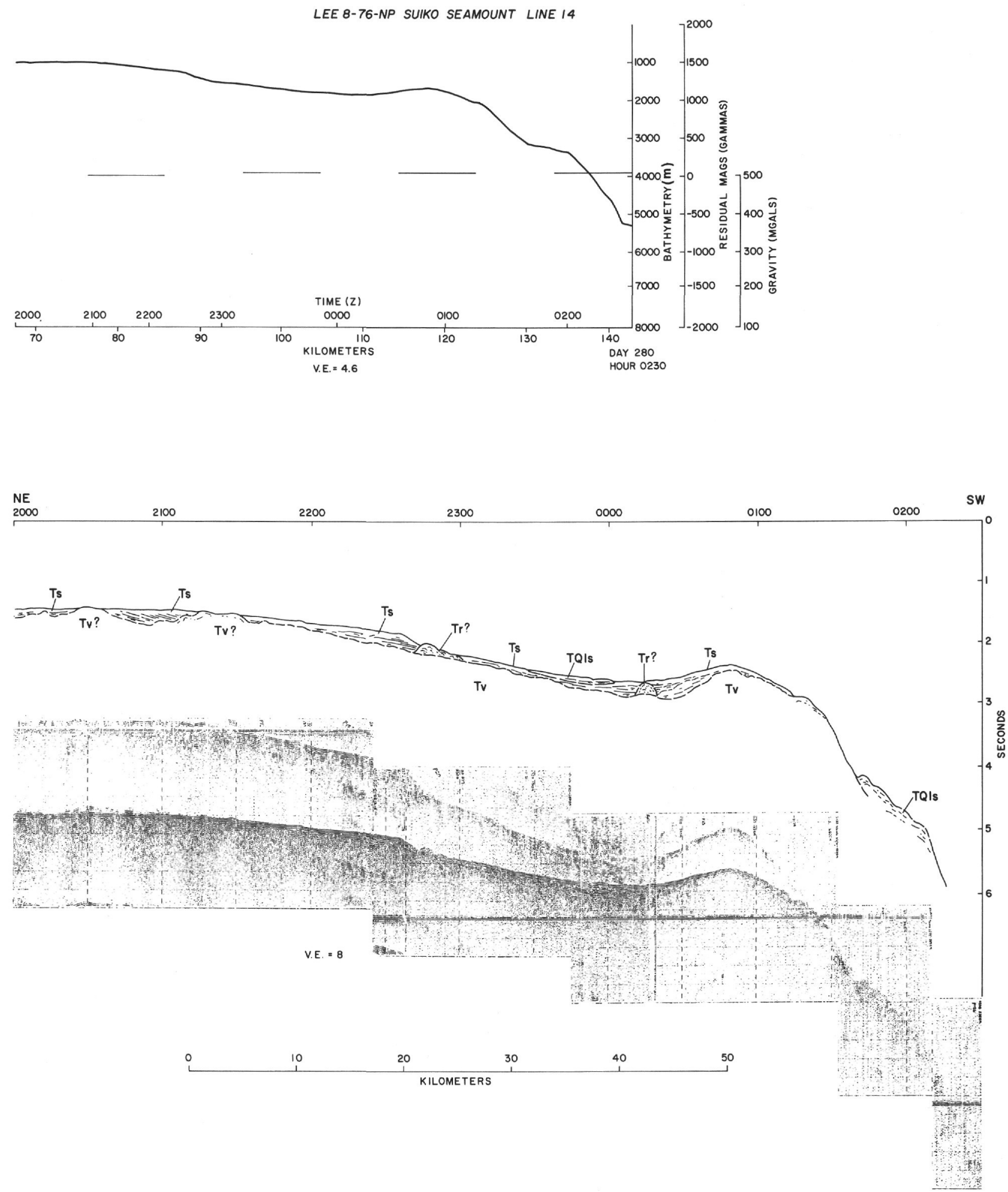

Figure 6. Continued. 

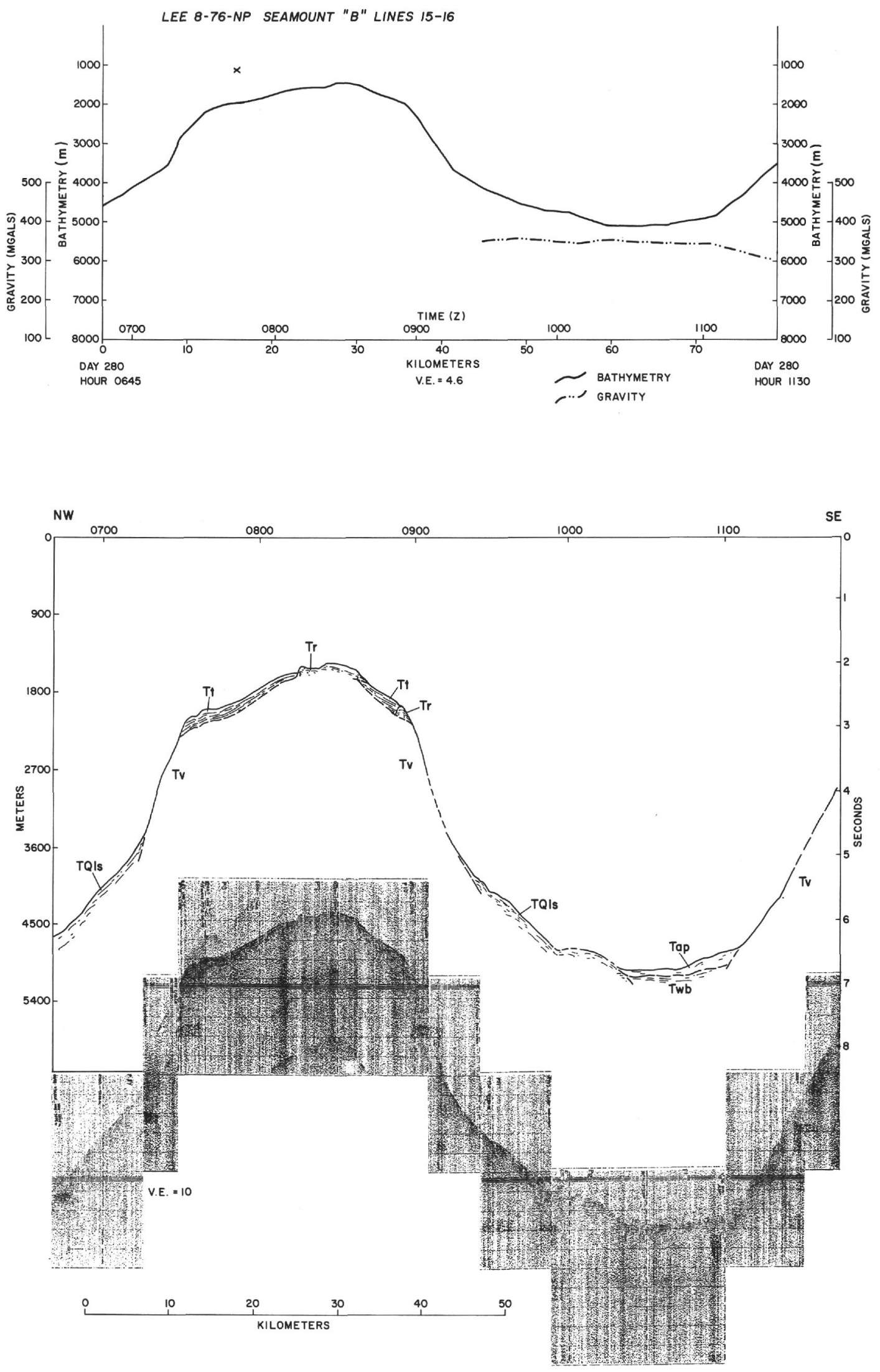

Figure 7. Geophysical profiles across an unnamed seamount (Seamount ' $B$ ") south of Suiko. Twb, Tertiary well-bedded sedimentary rocks; other symbols as in Figures 5 and 6. Location of profile shown in Figure 1. 

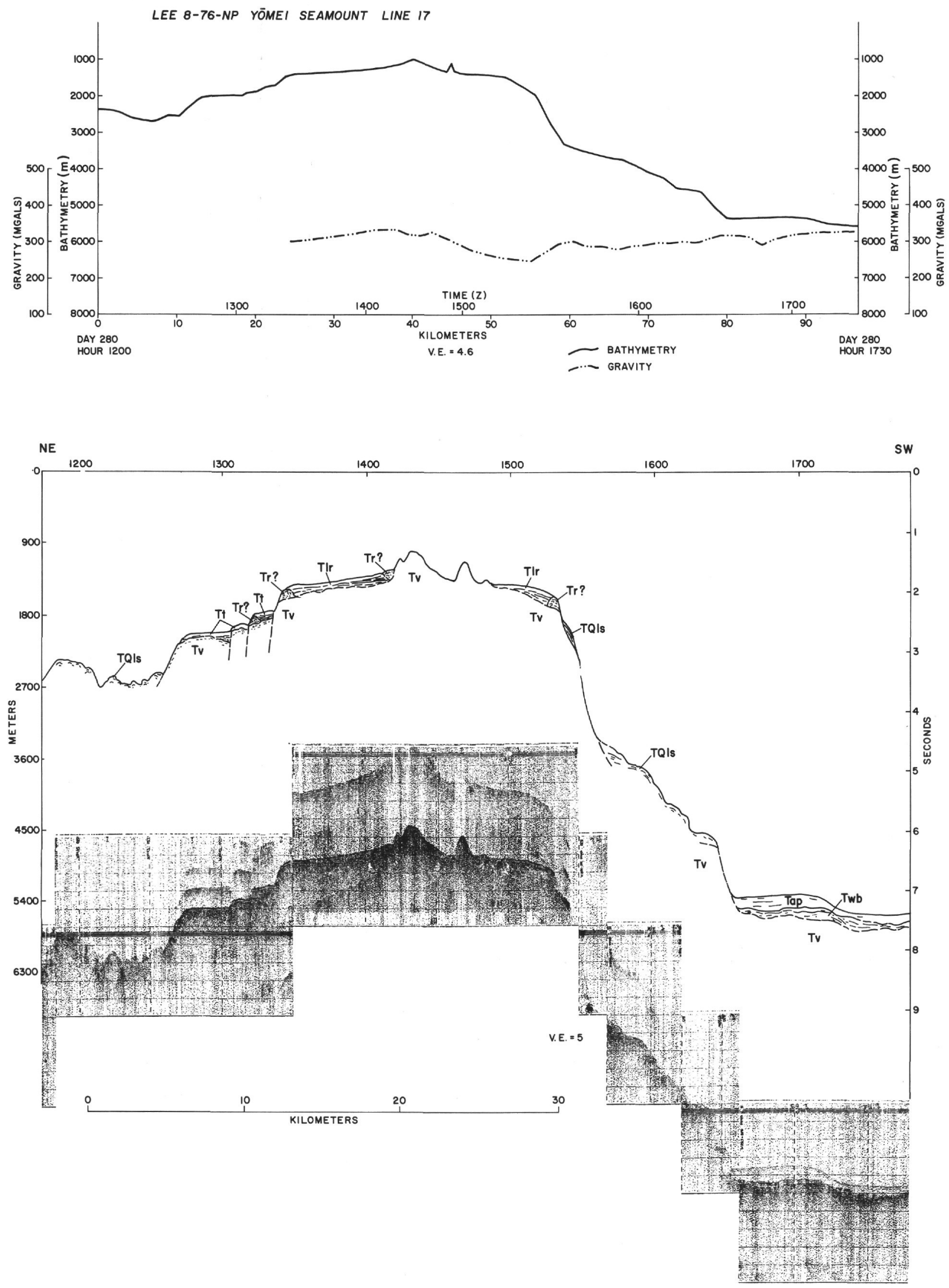

Figure 8. Geophysical profiles across Yōmei Seamount. Symbols as in Figures 5, 6, and 7. Location of profile shown in Figure 1. 

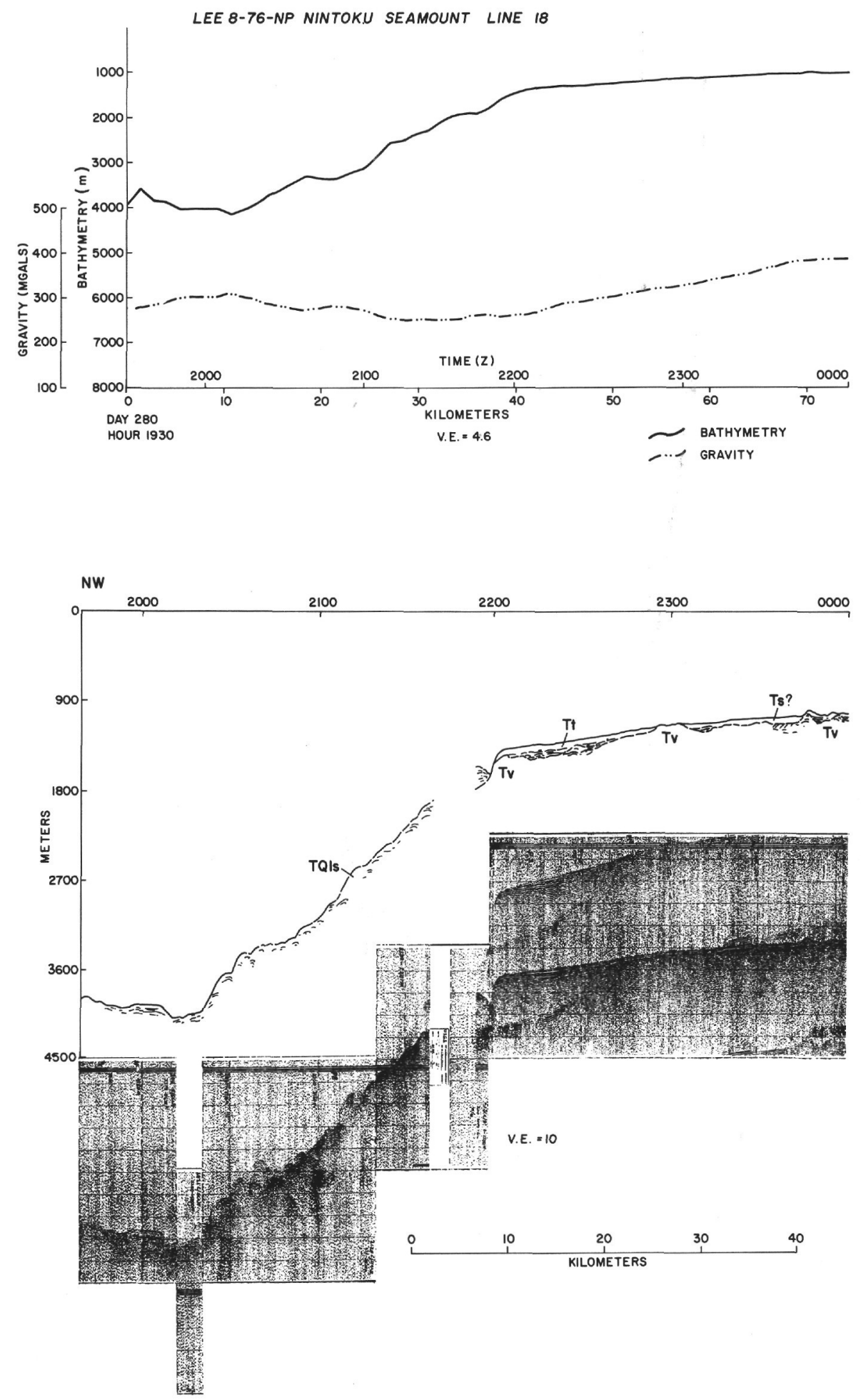

Figure 9. Geophysical profile across Nintoku Seamount. Symbols as in Figures 5, 6, and 7. Location of profile shown in Figure 1. 


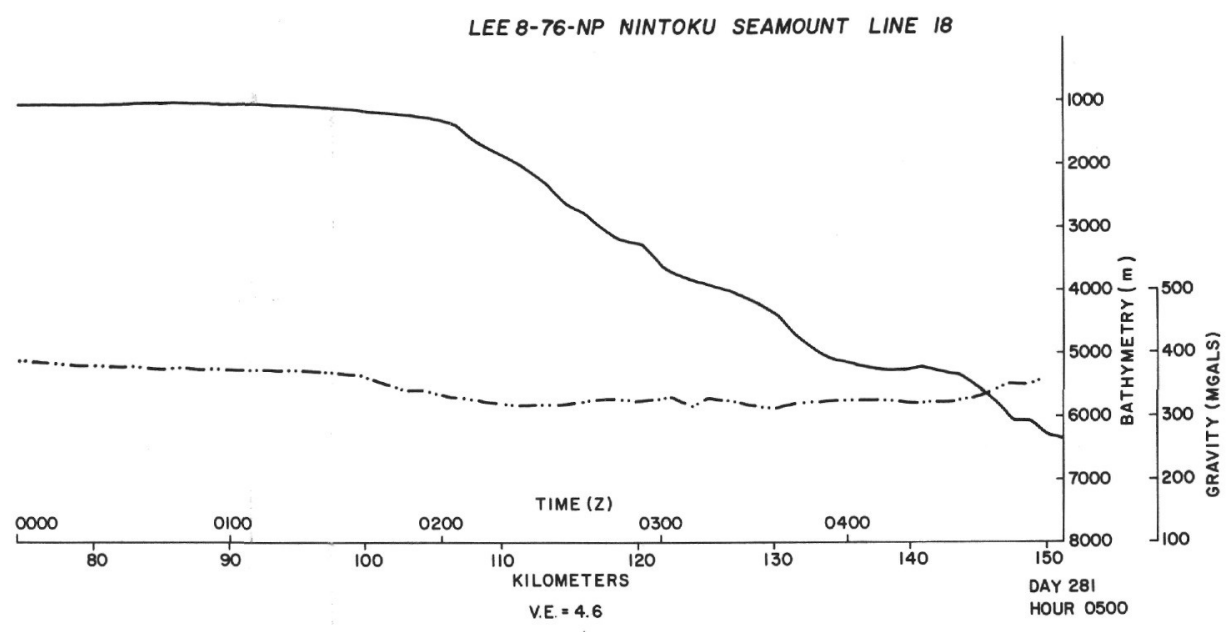

0000 0100 0200 0300 0400

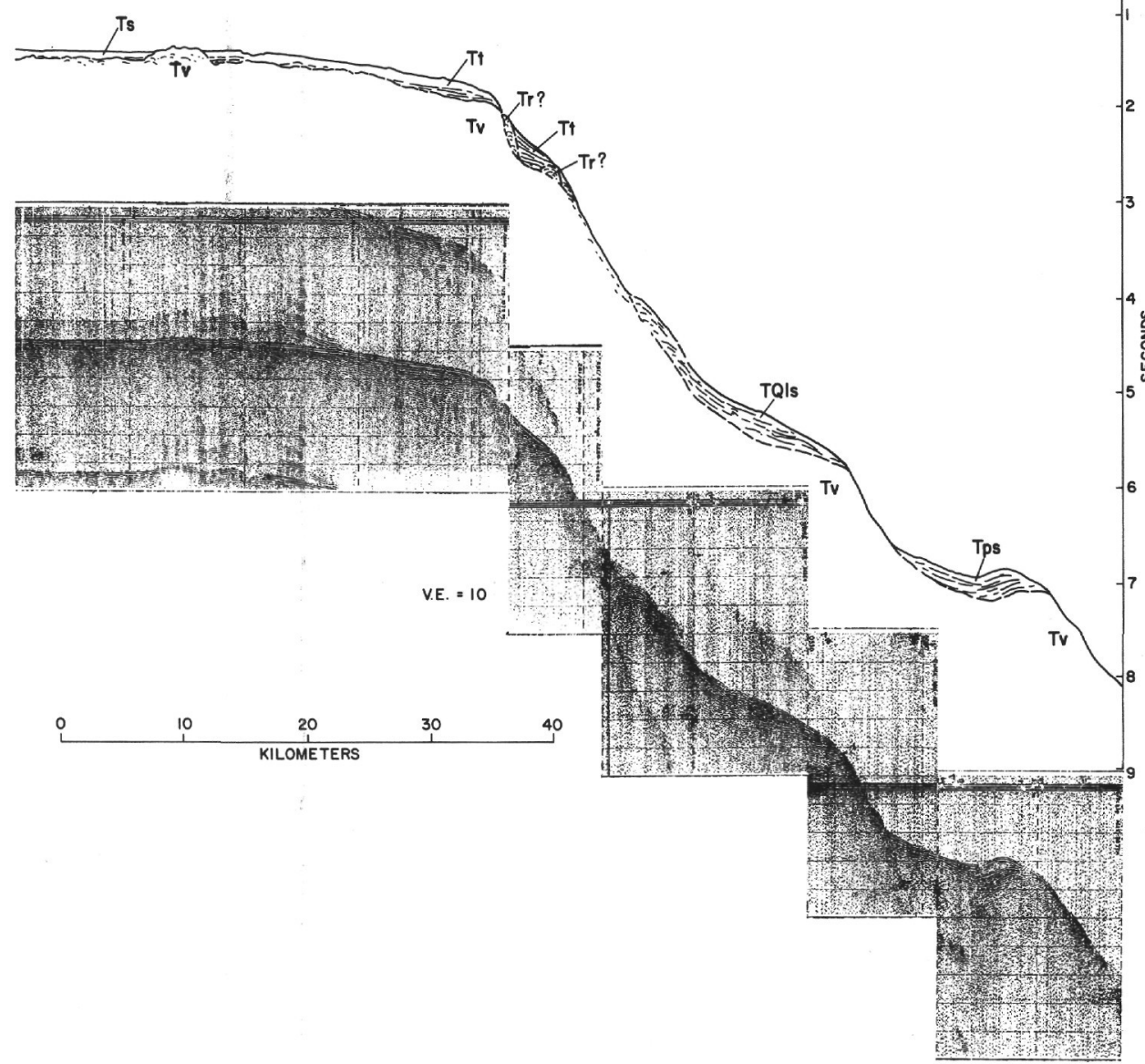

Figure 9. Continued. 

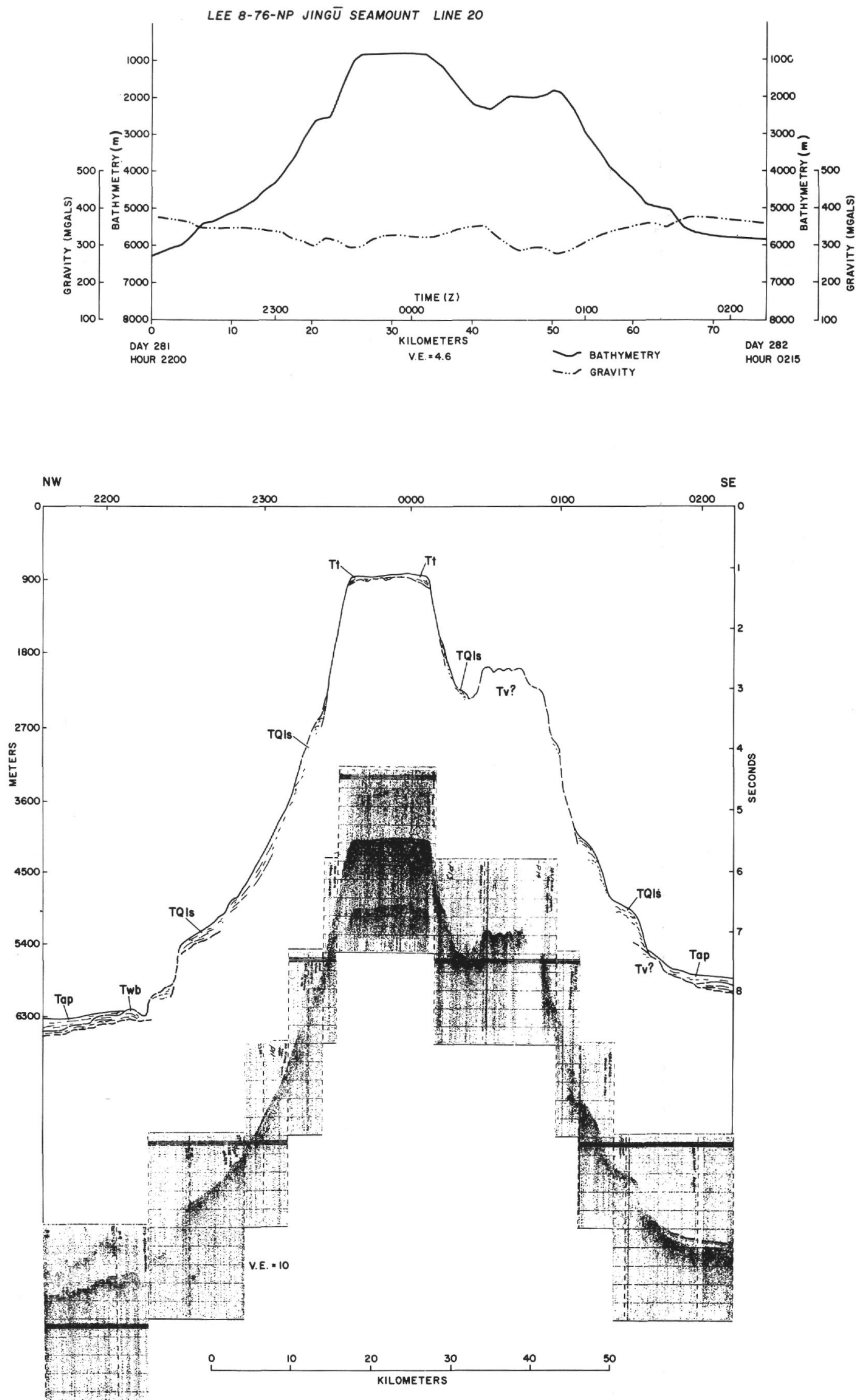

Figure 10. Geophysical profile across Jingū Seamount. Symbols as in Figures 5, 6, and 7. Location of profile shown in Figure 2. 
south edge of the seamount at $0700 \mathrm{Z}$ may be caused partly by topography and partly by vertical faulting at the inner edge of the reef. This anomaly has a shape typical of a block tilted slightly downward to the southeast. A 500-gamma negative anomaly near the north edge of the seamount at $0120 \mathrm{Z}$ represents the large-displacement vertical fault shown in the seismic reflection profile. The other magnetic anomalies are difficult to correlate with features in the seismic reflection profiles because of the lack of seismic penetration.

Conventionally, the Bouguer gravity reflects the excess mass of the volcanic rocks. There is some suggestion that the lower terrace on the southeast flank may be a southeast-dipping block.

\section{ACKNOWLEDGMENTS}

We thank Captain S. E. Keinanen, the officers, and the ship's and scientific crews for their assistance and support during the cruise. We also express our gratitude to A. G. McHendrie, R. Larsen, and B. R. Larsen for data processing, to J. V. Gardner and T. R. Brunes for manuscript review, and to D.
W. Scholl and E. C. Buffington and their staff, who made it all happen. The cruise was funded partly by NSF/IPOD through the Deep Sea Drilling Project.

\section{REFERENCES}

Chase, T. E., Menard, H. W., and Mammerickx, J., 1970. Bathymetry of the North Pacific, Charts 2, 7, and 8. La Jolla, Calif., Inst. Marine Resources, Scripps Inst. Oceanography.

Dalrymple, G. B., 1977. Shipboard observations of a north Pacific storm, Mariners Weather Log, v. 21, pp. 302-304.

Davies, T. A., Wilde, P., and Clague, D. A., 1972. Kōkō Seamount: A major guyot at the southern end of the Emperor Seamounts, Marine Geol., v. 13, pp. 311-321.

Greene, H. G., Dalrymple, G. B., and Clague, D. A., 1978. Evidence for northward movement of the Emperor Seamounts, Geology, v. 6, pp. 70-74.

Kodama, K., Uyeda, S., Isezaki, N., 1978. Paleomagnetism of Suiko Seamount, Emperor Seamount Chain, Geophys. Res. Lett., v. 5, pp. 165-168.

Malahoff, A. 1969. Gravity anomalies over volcanic regions. In Hart, P. J. (Ed.), The Earth's Crust and Upper Mantle: Am. Geophys. Monograph 13, pp. 364-379. 

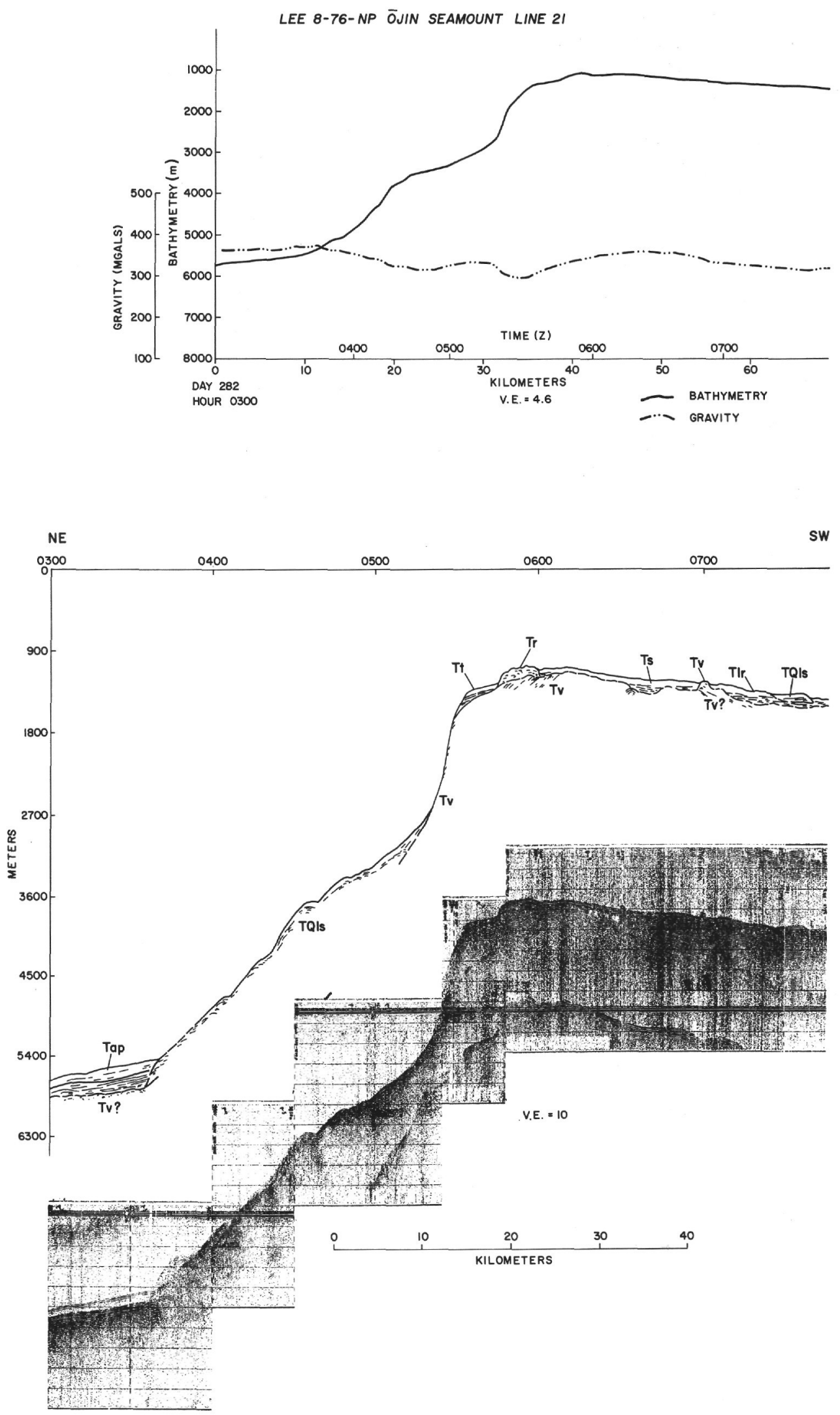

Figure 11. Geophysical profile across Ōjin Seamount. Symbols as in Figures 5, 6, and 7. Location of profile shown in Figure 2. 

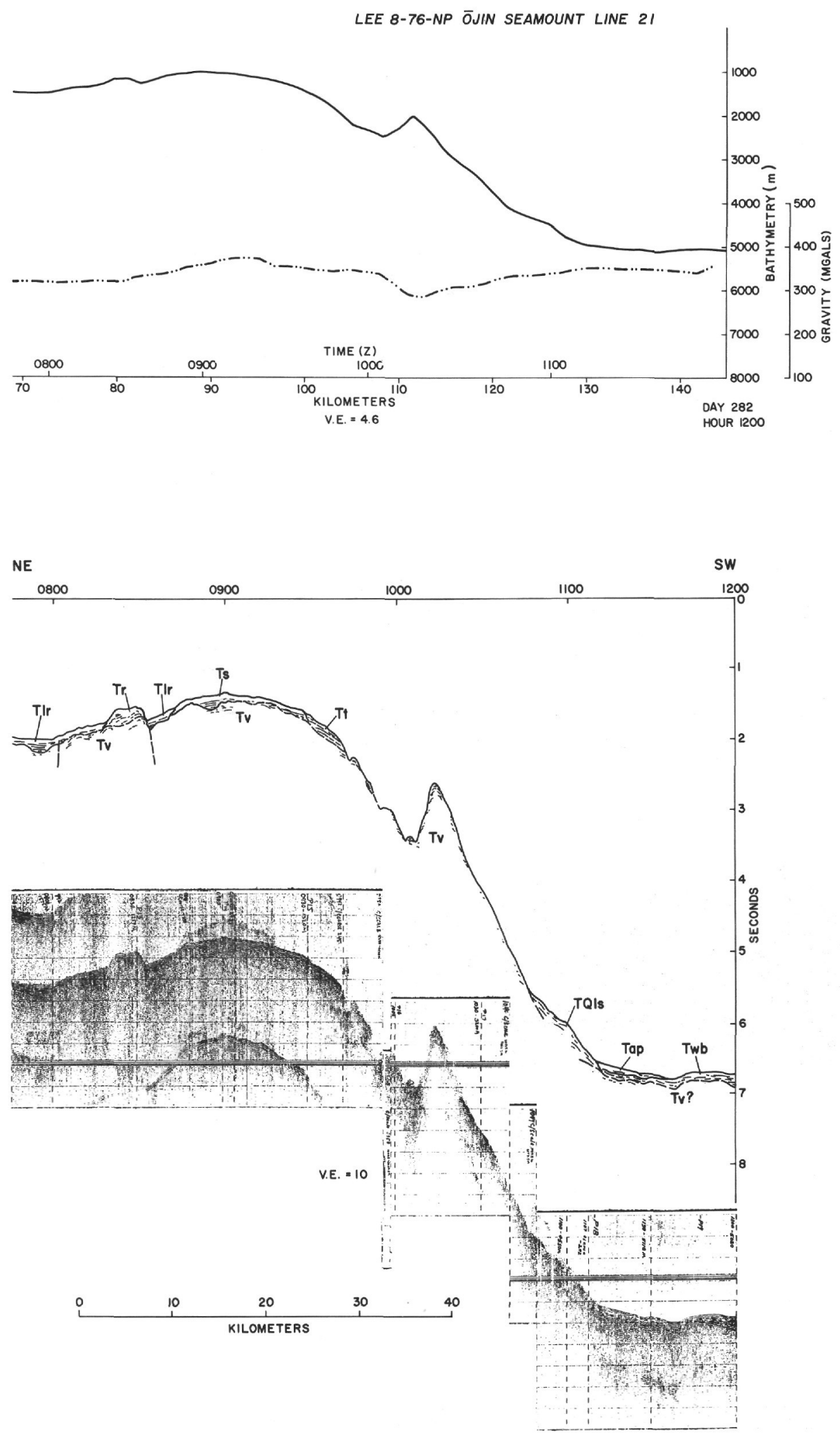

Figure 11. Continued. 

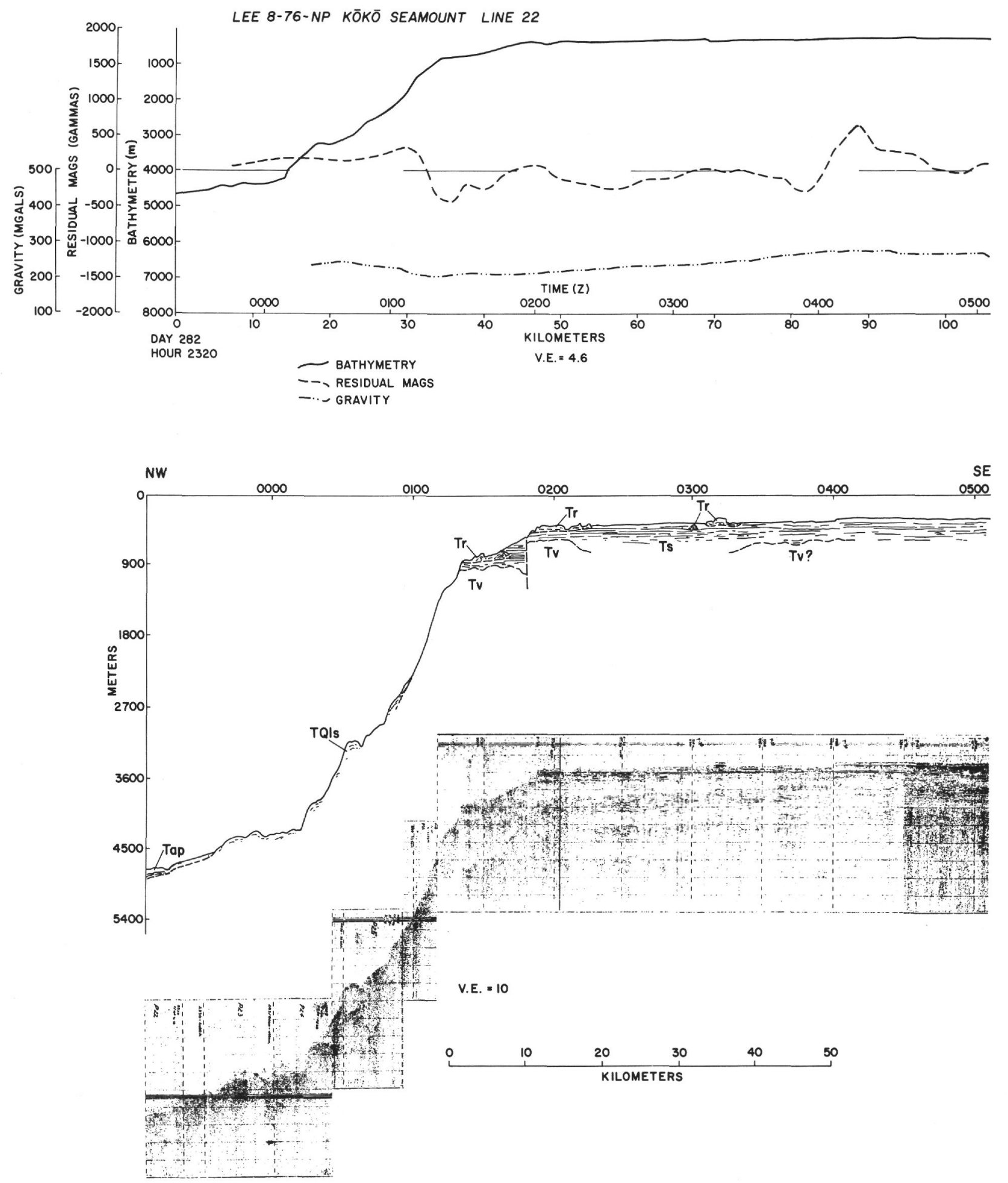

Figure 12. Geophysical profile across Kōkō Seamount. Symbols as in Figures 5, 6, and 7. Location of profile shown in Figure 2. 

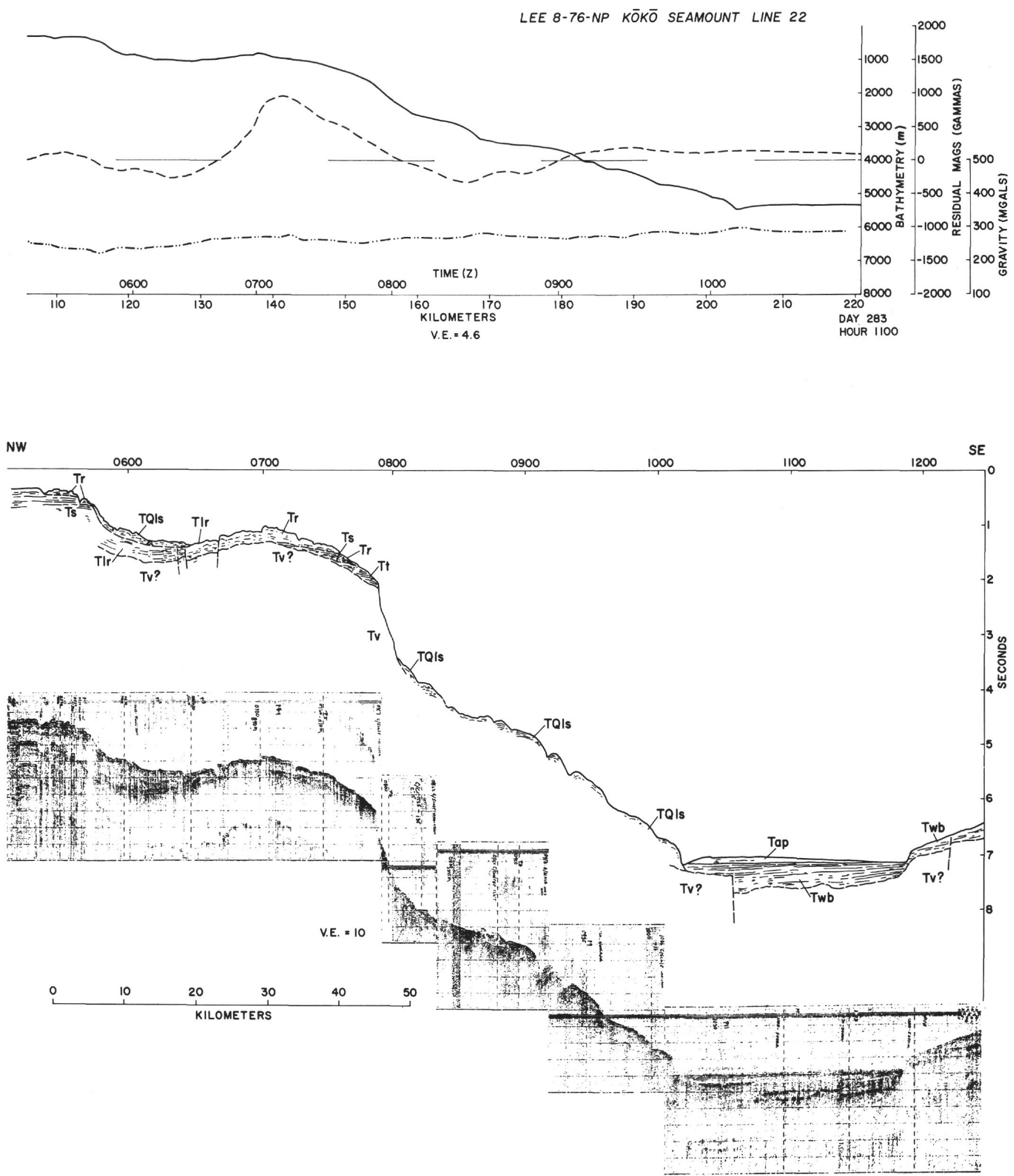

Figure 12. Continued. 